University of Tennessee Health Science Center

UTHSC Digital Commons

$12-2008$

\title{
ABCB6 Is a Porphyrin Transporter with a Novel Trafficking Signal That Is Conserved in Other ABC Transporters
}

Yu Fukuda

University of Tennessee Health Science Center

Follow this and additional works at: https://dc.uthsc.edu/dissertations

Part of the Chemicals and Drugs Commons, and the Medical Sciences Commons

\section{Recommended Citation}

Fukuda, Yu , "ABCB6 Is a Porphyrin Transporter with a Novel Trafficking Signal That Is Conserved in Other ABC Transporters" (2008). Theses and Dissertations (ETD). Paper 345. http://dx.doi.org/10.21007/ etd.cghs.2008.0100.

This Dissertation is brought to you for free and open access by the College of Graduate Health Sciences at UTHSC Digital Commons. It has been accepted for inclusion in Theses and Dissertations (ETD) by an authorized administrator of UTHSC Digital Commons. For more information, please contact jwelch30@uthsc.edu. 


\title{
ABCB6 Is a Porphyrin Transporter with a Novel Trafficking Signal That Is Conserved in Other ABC Transporters
}

\author{
Abstract \\ The ATP-binding cassette $(A B C)$ transporters play an important role as a barrier to protect cells from the \\ accumulation of toxic xenobiotics and metabolites due to their ability to translocate a wide array of \\ compounds across lipid bilayers. However, many ABC transporters, especially the ones localized in the \\ intracellular organelles, are involved in critical biological processes such as antigen presentation. The \\ core unit of $A B C$ transporters contains two functional domains: the membrane spanning domain (MSD) \\ and the nucleotide binding domain. The full transporters contain two of these units in tandem in a single \\ polypeptide, whereas the half transporters only contain one and must homo- or hetero-dimerize in order to \\ exert their functions. A half transporter ABCB6 has been shown to localize in mitochondria and suggested \\ to play a role in iron homeostasis; however, its function remained elusive. Therefore, we aimed to \\ characterize several aspects of ABCB6: identification of substrates, physiological role, transport \\ mechanism and intracellular trafficking. In this study, we show that $A B C B 6$ is a homodimeric \\ mitochondrial outer membrane protein. Furthermore, we identified $A B C B 6$ as a porphyrin transporter that \\ facilitates heme biosynthesis, in which the intermediates must be shuttled between mitochondria and the \\ cytosol. Translocation of substrates by ABC transporters occurs in an ATP-dependent manner, although \\ the role of ATP binding and hydrolysis in the transport process remains controversial. Taking advantage \\ of its ability to bind hemin conjugated to agarose beads, we demonstrate that the ATP binding at the NBD \\ is sufficient to induce a conformational change to a low affinity state in ABCB6. In an attempt to \\ understand how ABCB6 trafficks intracellularly, we identified a post-translational modification that \\ indicates ER to Golgi trafficking during its maturation. Moreover, we identified a novel N-terminal disulfide \\ bond that plays an important role in the ER exit of $A B C B 6$. This disulfide bond motif is found in other $A B C$ \\ family members and the loss of the conserved cysteine residue in ABCC8/SUR1 is the genetic basis for \\ hyperinsulinemic hypoglycemia. Because ER redox status appears to play an important role in the \\ trafficking of these proteins, expression patterns of $A B C$ transporters may be altered in \\ pathophysiological conditions such as diabetes where microsomal redox status is shifted.

\section{Document Type} \\ Dissertation \\ Degree Name \\ Doctor of Philosophy $(\mathrm{PhD})$ \\ Program \\ Interdisciplinary Program \\ Research Advisor \\ John D. Schuetz, Ph.D. \\ Keywords \\ $A B C$ transporters, $A B C B 6$, mitochondria, porphyrin, post-translational modification \\ Subject Categories \\ Chemicals and Drugs | Medical Sciences | Medicine and Health Sciences
}




\title{
ABCB6 IS A PORPHYRIN TRANSPORTER WITH A NOVEL TRAFFICKING SIGNAL THAT IS CONSERVED IN OTHER ABC TRANSPORTERS
}

\author{
A Dissertation \\ Presented for \\ The Graduate Studies Council \\ The University of Tennessee \\ Health Science Center
}

\author{
In Partial Fulfillment \\ Of the Requirements for the Degree \\ Doctor of Philosophy \\ From The University of Tennessee
}

By

Yu Fukuda

December 2008 
Portions of Chapter 2 (C) 2006 by Nature Publishing Group All other material @ 2008 by Yu Fukuda All rights reserved 


\section{DEDICATION}

I would like to dedicate this dissertation to my parents, Toshio and Etsuko Fukuda for nurturing me with constant love and support so that my roots are firmly grounded to withstand any weather. 


\section{ACKNOWLEDGMENTS}

I would like to acknowledge and thank everyone who contributed to my dissertation work in the past years. First and foremost I would like to thank my research advisor, Dr. John Schuetz, for his willingness to take me as his first graduate student, his patience, his guidance and his trust. He taught me to stay focused on important issues and encouraged me to develop as a scientist. I would like to also thank all the past and present members of the Schuetz lab for their help, friendship and support. A special thanks to Dr. Partha Krishnamurthy for all the advice he has given me and for all the discussions we had together. I would like to also thank Jessie Morgan for her continuous support and for teaching me the 'Southern' styles. I would like to thank Dr. Daxi Sun for helping me with plasmid construction initially, Yao Wang for FACS analysis and Dr. Guoqing Du for plasmids. I also like to thank Bath student, Dan Martin, for repeating the pull-down assays. I would also like to extend my gratitude to Dr. Erin Schuetz for her helpful and insightful comments during the lab meetings.

I would also like to thank my graduate committee, Dr. Linda Hendershot, Dr. Jim Morgan, Dr. A. P. Naren and Dr. Jie Zheng for taking part in my graduate training, their guidance and their commitment.

I would also like to thank Dr. Jufang Shan for homology modeling help, Yi Jin for showing me how to do pulse chase studies, and Dr. Joseph Bryan at Pacific Northwest Diabetes Research Institute for his contribution to the SUR1 experiment. I would also like to thank Flow cytometry lab for running PPIX measurements. A special thanks to fellow graduate students especially my classmates for their support and for their friendship.

I would like to thank Dr. Louise Treanor for her friendship. I would like to thank my best friend Patrick Siglin for his continuous support and for motivating me to be the best person I can be.

I would also like to express my sincere thanks to Dr. Yasuyuki Igarashi, Dr. Akio Kihara and Dr. Gabor Tigyi for their support and for encouraging me to pursue my doctorate degree in the United States.

Last, but certainly not least, I would like to thank my family and friends. Without their support, none of this would have been possible. 


\begin{abstract}
The ATP-binding cassette (ABC) transporters play an important role as a barrier to protect cells from the accumulation of toxic xenobiotics and metabolites due to their ability to translocate a wide array of compounds across lipid bilayers. However, many $\mathrm{ABC}$ transporters, especially the ones localized in the intracellular organelles, are involved in critical biological processes such as antigen presentation. The core unit of ABC transporters contains two functional domains: the membrane spanning domain (MSD) and the nucleotide binding domain. The full transporters contain two of these units in tandem in a single polypeptide, whereas the half transporters only contain one and must homo- or hetero-dimerize in order to exert their functions. A half transporter ABCB6 has been shown to localize in mitochondria and suggested to play a role in iron homeostasis; however, its function remained elusive. Therefore, we aimed to characterize several aspects of ABCB6: identification of substrates, physiological role, transport mechanism and intracellular trafficking. In this study, we show that ABCB6 is a homodimeric mitochondrial outer membrane protein. Furthermore, we identified ABCB6 as a porphyrin transporter that facilitates heme biosynthesis, in which the intermediates must be shuttled between mitochondria and the cytosol. Translocation of substrates by ABC transporters occurs in an ATP-dependent manner, although the role of ATP binding and hydrolysis in the transport process remains controversial. Taking advantage of its ability to bind hemin conjugated to agarose beads, we demonstrate that the ATP binding at the NBD is sufficient to induce a conformational change to a low affinity state in ABCB6. In an attempt to understand how ABCB6 trafficks intracellularly, we identified a post-translational modification that indicates ER to Golgi trafficking during its maturation. Moreover, we identified a novel N-terminal disulfide bond that plays an important role in the ER exit of ABCB6. This disulfide bond motif is found in other ABC family members and the loss of the conserved cysteine residue in ABCC8/SUR1 is the genetic basis for hyperinsulinemic hypoglycemia. Because ER redox status appears to play an important role in the trafficking of these proteins, expression patterns of $A B C$ transporters may be altered in pathophysiological conditions such as diabetes where microsomal redox status is shifted.
\end{abstract}




\section{TABLE OF CONTENTS}

CHAPTER 1. INTRODUCTION................................................................ 1

1.1. Historic Overview: Discovery of P-glycoprotein and ABC Protein Superfamily.... 1

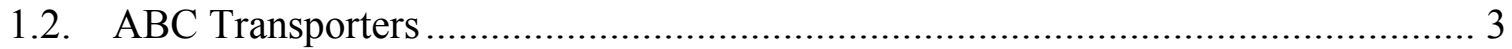

1.2.1. ABC Transporters and MDR Phenotypes ….................................... 7

1.2.2. Physiological Roles of ABC Transporters ............................................. 8

1.2.3. ABC Transporters and Diseases/Phenotypes ......................................11

1.2.4. Architecture of ABC Transporters..................................................... 11

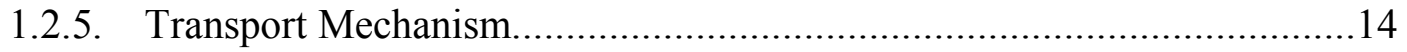

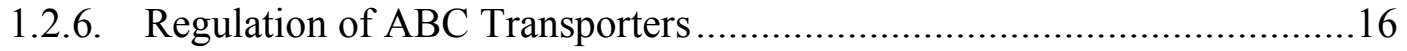

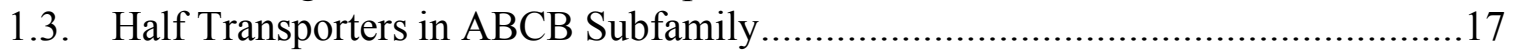

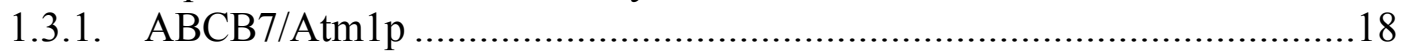

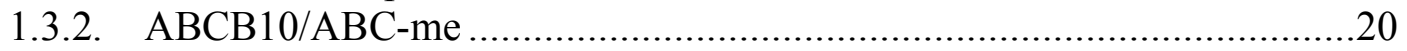

1.3.3. Identification of ABCB6 (PRP/UMAT/MTABC3) ................................20

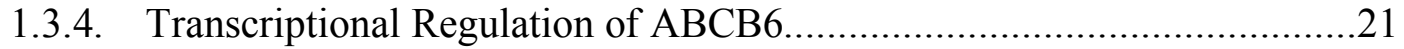

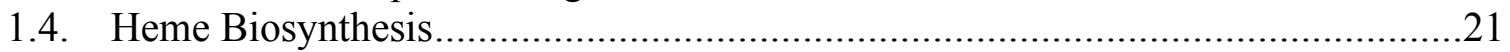

1.4.1. Heme Biosynthesis and Diseases.......................................................22

1.4.2. Compounds That Interfere with Heme Biosynthesis ............................24

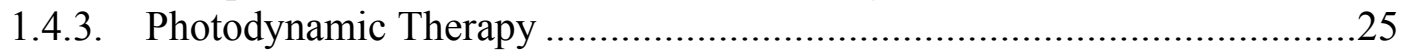

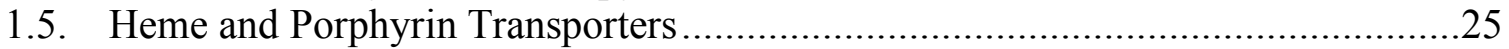

1.5.1. Heme Importer: Heme Carrier Protein 1 ..............................................26

1.5.2. Heme/Porphyrin Exporters.................................................................26

1.5.3. Intracellular Porphyrin Transporters....................................................27

CHAPTER 2. CHARACTERIZATION OF ABCB6 AS A PORPHYRIN TRANSPORTER.............................................................................29

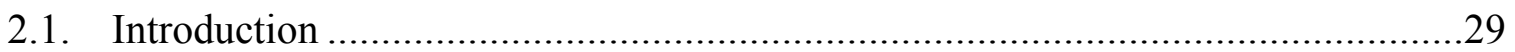

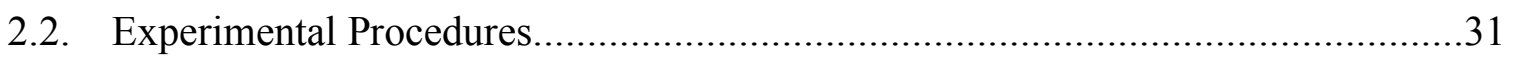

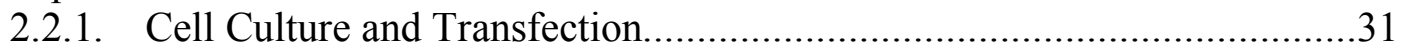

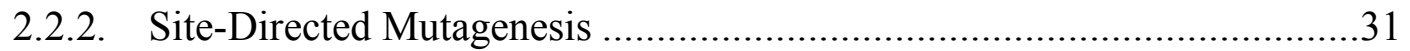

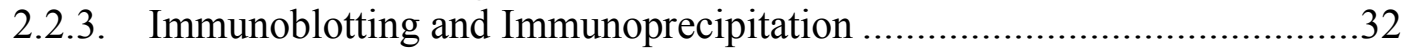

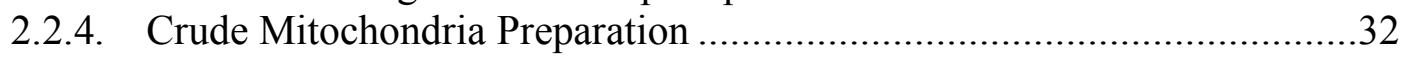

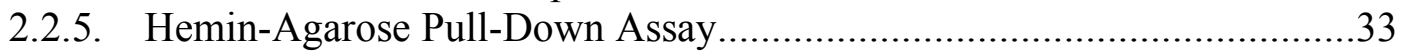

2.2.6. ATPase Activity Measurement...............................................................33

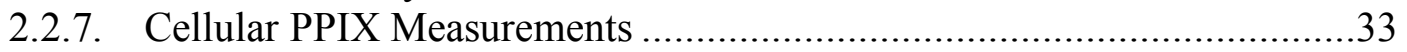

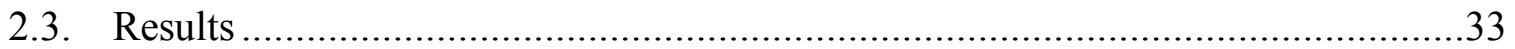

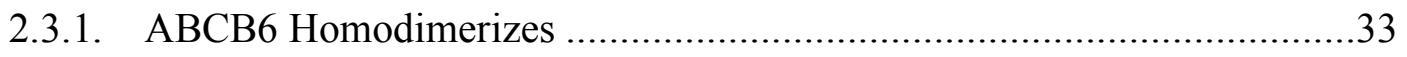

2.3.2. Interaction between ABCB6 and Heme Is Disrupted by Tetrapyrroles.....37

2.3.3. ABCB6 Transports Porphyrins in an ATP-Dependent Manner ................37

2.3.4. ABCB6 Increases the Rate of Porphyrin Biosynthesis ......................... 41

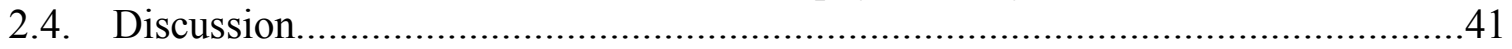




\section{CHAPTER 3. ABCB6 PROVIDES INSIGHTS INTO THE MECHANISM FOR}

SUBSTRATE TRANSPORT BY ABC TRANSPORTERS

3.1. Introduction

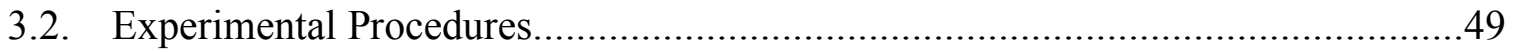

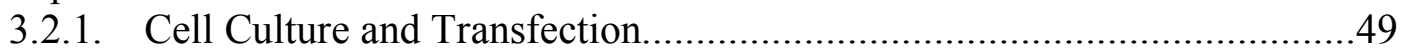

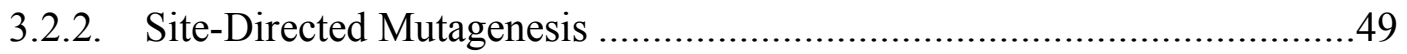

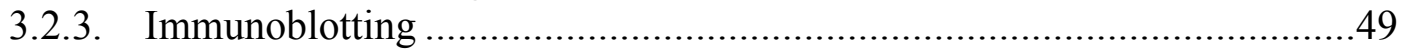

3.2.4. Hemin-Agarose or ATP-Agarose Pull-Down Assays..............................50

3.2.5. Nucleotide-Induced ABCB6 Dissociation ……....................................50

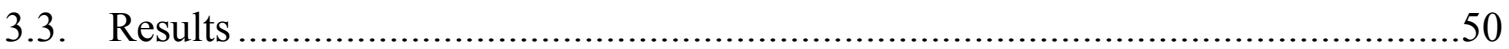

3.3.1. ABCB6 and ABCB6 ${ }^{\mathrm{K} 629 \mathrm{G}}$ Display Similar Kinetics for Hemin Binding ..50

3.3.2. Loss of the Walker A Lysine Results in Reduced ATP Binding...............51

3.3.3. ATP Binding to ABCB6 Changes Its Affinity for Hemin-Agarose ...........51

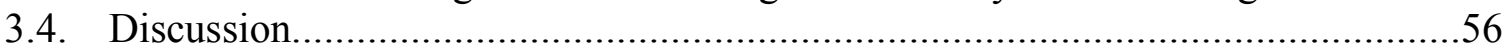

CHAPTER 4. ABCB6 CONTAINS AN N-TERMINAL ER EXIT SIGNAL THAT IS CONSERVED IN OTHER ABC TRANSPORTERS..............................60

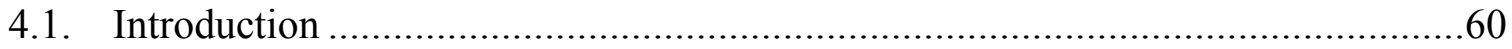

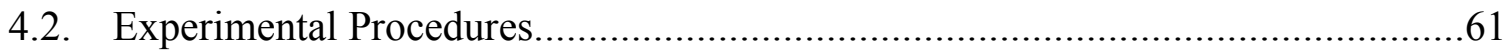

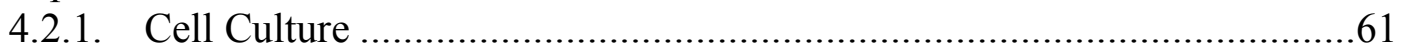

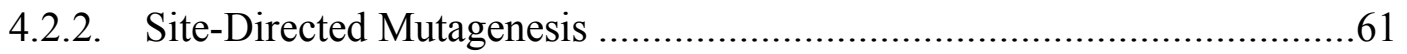

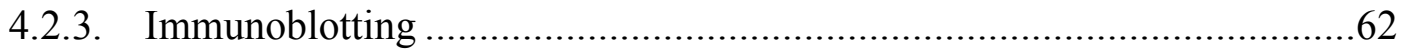

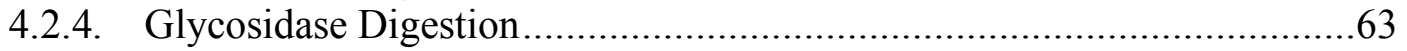

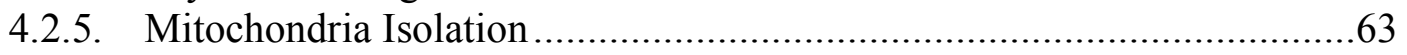

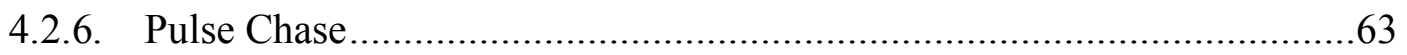

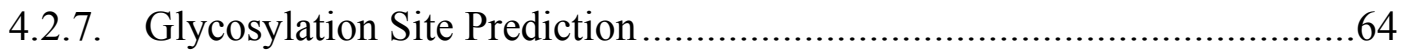

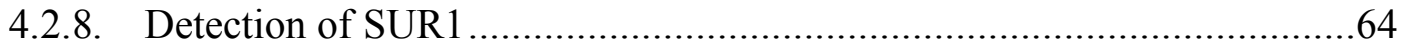

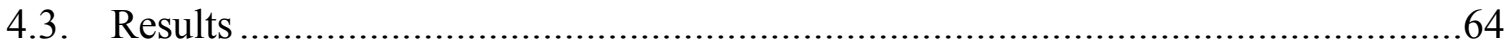

4.3.1. Both Endogenous and Exogenous ABCB6 Are Glycosylated ...................64

4.3.2. ABCB6 Is Glycosylated at an Atypical N-X-C Site .................................68

4.3.3. Cysteine Is Dispensable for Glycosylation but Is Critical for ER Exit......75

4.3.4. Cys8 and Cys26 Form Intramolecular Disulfide Bond.............................78

4.3.5. N-terminal Cysteine Residues Are Conserved in the Long MRPs.............78

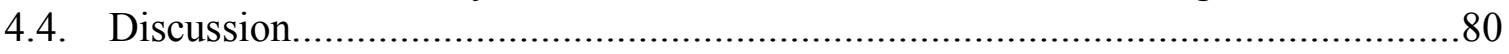

4.4.1. N-Terminal Disulfide Bond Regulates ER Exit of ABC Transporters......80

4.4.2. ABCB6 Is Processed in the ER and Golgi .............................................83

4.4.3. Physiological Implication of the N-Terminal Disulfide Bond ...................84

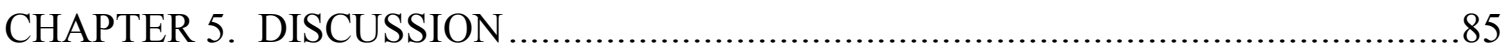

5.1. ABCB6 Is a Homodimeric Mitochondrial Porphyrin Transporter.........................86

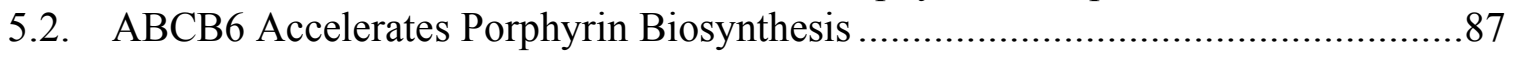

5.3. ATP Binding Releases the Substrate from ABCB6.............................................87

5.4. ABCB6 Is Glycosylated and Contains a Disulfide-Bonded Loop That Acts as a

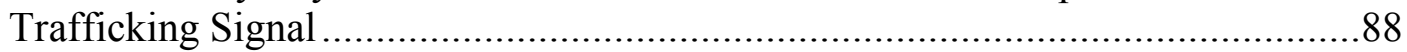

5.4.1. Formation of Disulfide Bond in ABCB6 Acts as an ER Exit Signal.........89

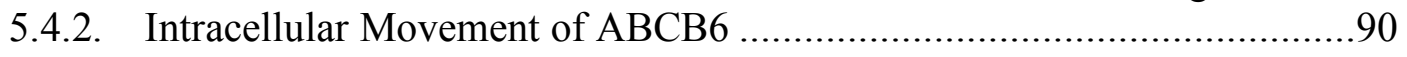




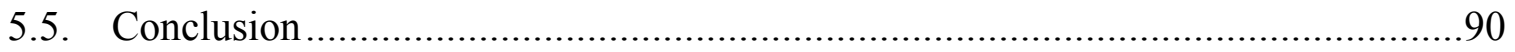

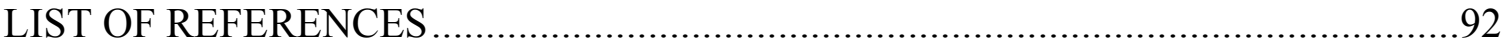

VITA 


\section{LIST OF FIGURES}

Figure 1.1. Schematic representations of $\mathrm{ABC}$ transporters......................................... 4

Figure 1.2. Interaction between ATP and different motifs from two NBDs ...................13

Figure 1.3. Bacterial ABC transporter Sav1866 structure ............................................15

Figure 1.4. Heme biosynthesis pathway ……………...............................................23

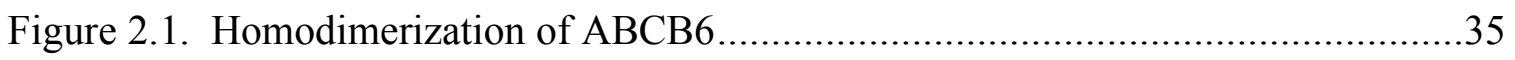

Figure 2.2. Mutation in the Walker A motif does not affect ABCB6 dimerization..........36

Figure 2.3. GXXXG and the $\mathrm{D}(\mathrm{X})_{8} \mathrm{D}$ domains are not critical for the dimerization ........38

Figure 2.4. Walker A mutant and GXXXG mutant bind hemin-agarose ........................39

Figure 2.5. CPIII, PPIX, and PhA displace ABCB6 from hemin-agarose ......................40

Figure 2.6. CPIII stimulates ATPase activity in ABCB6 expressing mitochondria ........42

Figure 2.7. ABCB6 expressing cells exhibit higher intracellular PPIX levels ................43

Figure 3.1. $\mathrm{ABCB} 6$ and $\mathrm{ABCB} 6^{\mathrm{K} 629 \mathrm{G}}$ binds to hemin-agarose with similar kinetics .......52

Figure 3.2. ATP-binding is severely impaired in Walker A mutant ABCB6 ……..........53

Figure 3.3. Binding of nucleotides to $\mathrm{ABCB} 6$ changes affinity for hemin-agarose ........57

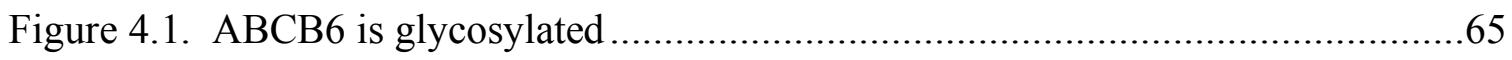

Figure 4.2. ABCB6 is glycosylated only at the atypical N-X-C site..............................69

Figure 4.3. ABCB6 is predicted to contain 9 TM helices...............................................

Figure 4.4. Cys8 is dispensable for glycosylation but is critical for ABCB6 ER exit .....76

Figure 4.5. Both Cys8 and Cys26 are required for ER exit of ABCB6..........................77

Figure 4.6. N-terminus of $\mathrm{ABCB} 6$ contains a disulfide bond........................................79

Figure 4.7. N-terminal cysteines are conserved in long MRPs and regulate trafficking..81 


\section{LIST OF ABBREVIATIONS}

\begin{tabular}{|c|c|}
\hline $\mathrm{ABC}$ & ATP binding cassette \\
\hline ALA & 5-aminolevulinate \\
\hline ALAD & ALA dehydratase \\
\hline ALAS & ALA synthase \\
\hline ATP & adenosine triphosphate \\
\hline $\mathrm{BBB}$ & blood-brain barrier \\
\hline $\mathrm{BCF}$ & blood-cerebrospinal fluid barrier \\
\hline BCRP & breast cancer resistance protein \\
\hline cAMP & cyclic adenosine monophosphate \\
\hline CAR & constitutive androstane receptor \\
\hline $\mathrm{CF}$ & cystic fibrosis \\
\hline CFTR & cystic fibrosis transmembrane conductance regulator \\
\hline CNS & central nervous system \\
\hline CPIII & coproporphyrin III \\
\hline CPgenIII & coproporphyrinogen III \\
\hline $\mathrm{CSF}$ & cerebrospinal fluid \\
\hline DMSO & dimethylsulfoxide \\
\hline ER & endoplasmic reticulum \\
\hline EST & expressed sequence tag \\
\hline FXR & farnesoid X receptor \\
\hline GFP & green fluorescent protein \\
\hline GPCR & G protein coupled receptors \\
\hline GSH & glutathione \\
\hline HCP1 & heme carrier protein 1 \\
\hline HIF-1 & hypoxia-inducible factor 1 \\
\hline HMT & heavy metal tolerance- 1 \\
\hline HUGO & Human Genome Organization \\
\hline IRE & iron responsive element \\
\hline IRP & iron regulatory protein \\
\hline $\mathrm{kb}$ & kilobase \\
\hline $\mathrm{kDa}$ & kilo Dalton \\
\hline $\mathrm{KO}$ & knockout \\
\hline MDR & multidrug resistance \\
\hline Mel & murine erythroleukemia \\
\hline MFS & major facilitator superfamily \\
\hline $\mathrm{MHC}$ & major histocompatibility complex \\
\hline MRP & multidrug resistance-associated protein \\
\hline MSD & membrane spanning domains \\
\hline NBD & nucleotide binding domain \\
\hline NRF1 & nuclear respiratory factor-1 \\
\hline OGC & 2-oxoglutarate carrier \\
\hline PBG & porphobilinogen \\
\hline PBGD & PBG deaminase \\
\hline
\end{tabular}




$\begin{array}{ll}\text { PBR } & \text { peripheral-type benzodiazepine receptor } \\ \text { PBS } & \text { phosphate-buffered saline } \\ \text { P-gp } & \text { P glycoprotein } \\ \text { PhA } & \text { pheohorbide a } \\ \text { PPARg } & \text { peroxisome proliferator-activated receptor gamma } \\ \text { PPIX } & \text { protoporphyrin IX } \\ \text { PXR } & \text { pregnane X receptor } \\ \text { SA } & \text { succinyl acetone } \\ \text { SDS-PAGE } & \text { SDS-polyacrylamide gel electrophoresis } \\ \text { siRNA } & \text { small interfering RNA } \\ \text { SLC } & \text { solute carrier } \\ \text { SNP } & \text { single nucleotide polymorphism } \\ \text { SUR } & \text { sulfonylurea receptor } \\ \text { TAP } & \text { transporters associated with antigen processing } \\ \text { TAPL } & \text { TAP-like } \\ \text { TM } & \text { transmembrane } \\ \text { TNM } & \text { tunicamycin } \\ \text { UROD } & \text { uroporphyrinogen decarboxylase } \\ \text { UROS } & \text { uroporphyrinogen III synthase } \\ \text { XLSA/A } & \text { X-linked sideroblastic anemia and ataxia }\end{array}$




\section{CHAPTER 1. INTRODUCTION}

\subsection{Historic Overview: Discovery of P-glycoprotein and ABC Protein Superfamily}

... in resistant cells there is an outward flow of daunomycin which is against an electrochemical gradient, carrier mediated, and dependent on the energy metabolism.

Dano, K., Active outward transport of daunomycin in resistant Ehrlich ascites tumor cell, p. 480 [1].

The adenosine triphosphate (ATP)-binding cassette ( $\mathrm{ABC}$ ) transporters in the $\mathrm{ABC}$ protein superfamily translocate various molecules across lipid bilayers in an energy-dependent manner. Because of their wide array of substrates, genetic variations in $\mathrm{ABC}$ transporters are now known to be involved in the pathophysiology of numerous human disorders (Table 1.1.)[2]. ABC proteins comprise one of the largest protein superfamilies with 48 members identified to date in humans. The first mammalian $\mathrm{ABC}$ protein was discovered in 1976 as a $170-\mathrm{kDa}$ membrane glycoprotein that was overexpressed in colchicine resistant cell lines [3]. These cells were cross-resistant to different classes of drugs, and it was speculated that this protein altered drug permeability at the membrane resulting in a lower accumulation of drugs in resistant cells. Thus, this protein was named $\mathrm{P}$ glycoprotein $(\mathrm{P}-\mathrm{gp})$ for glycoprotein that reduces drug permeability. Many had suspected early on that multidrug resistance (MDR) would be a major obstacle in treating complex diseases such as cancer. By 1960's, several groups found a correlation between drug accumulation and drug response [4-6]; however, the uptake (influx) and efflux of drugs were not discriminated, and they simply speculated that the rate of uptake was altered in the drug resistant cells. In 1973, Dano provided definitive evidence of active efflux of daunomycin by measuring the steady state intracellular and extracellular drug accumulation in parental and daunomycin resistant cell lines [1]. Also, a metabolic inhibitor, $\beta$-deoxyglucose, was shown to inhibit the efflux of daunomycin. From these experiments, Dano concluded that "in resistant cells there is an outward flow of daunomycin which is against an electrochemical gradient, carrier mediated, and dependent on the energy metabolism" [1], which accurately predicted the function of yet to be identified $\mathrm{ABC}$ transporters.

While Ling and his colleagues observed P-gp gene ( 4.7 kilobases $(\mathrm{kb})$ ) amplification in different MDR mammalian cell lines and were determined to identify the role of P-gp in MDR [7], Roninson and colleagues identified and isolated DNA sequences that were commonly amplified in several MDR cell lines [8]. Of the many amplified DNA sequences observed, one such region, $m d r$, was isolated, which was found to contain a gene that transcribed $\sim 5 \mathrm{~kb}$ mRNA [9]. Gros and the colleagues then went on to show that the ectopic expression of mouse $m d r l$ cDNA conferred MDR in drug-sensitive Chinese Hamster cells [10]. However, the relationship between P-gp and $m d r$ was not clear at this point. Using P-gp specific antibody and cross-hybridization of P-gp probe and $m d r l \mathrm{cDNA}$, Ueda et al. finally showed that the mdr 1 gene product is Pgp [11]. It became clear from different approaches to identify mechanisms of MDR phenotypes that P-gp, the product of $m d r l$, was one of the key players in MDR. 
Table 1.1. Diseases and phenotypes caused by mutations or a lack of $A B C$ genes.

\begin{tabular}{lll}
\hline Gene & Mendelian disorder/phenotype & Reference \\
\hline ABCA1 & Tangier disease, FHDLD & {$[12-16]$} \\
ABCA3 & Lung Surfactant deficiency & {$[17]$} \\
ABCA4 & Stargards/FFM, RP, CRD & {$[18,19]$} \\
ABCA12 & Lamellar and harlequin ichthyosis & {$[20]$} \\
ABCB1 & Ivermectin sensitivity (in collie dogs) & {$[21,22]$} \\
ABCB2 & Immune deficiency & {$[23,24]$} \\
ABCB3 & Immune deficiency & {$[23,24]$} \\
ABCB4 & PFIC-3 & {$[25]$} \\
ABCB7 & XLSA/A & {$[26]$} \\
ABCB11 & PFIC-2 & {$[27]$} \\
ABCC2 & Dubin-Johnson syndrome & {$[28]$} \\
ABCC6 & Pseudoxanthoma elasticum & {$[29]$} \\
ABCC7 & Cystic fibrosis & {$[30]$} \\
ABCC8 & FPHHI & {$[31]$} \\
ABCC9 & DCVT & {$[32]$} \\
ABCC11 & Dry ear wax & {$[33]$} \\
ABCD1 & ALD & {$[34]$} \\
ABCG2 & Porphyria (observed in mouse) & {$[35]$} \\
ABCG5 & Sitosterolemia & {$[36]$} \\
ABCG8 & Sitosterolemia & {$[36]$} \\
\hline
\end{tabular}

Abbreviations used are ALD, adrenoleukodystrophy; CRD, cone-rod dystrophy; DCVT, dilated cardiomyopathy with ventricular tachycardia; FFM, fundus flavimaculatus; FHDLD, familial hypoapoproteinemia; FPHHI, familial persistent hyperinsulinemic hypoglycemia of infancy; PFIC, progressive familial intrahepatic cholestasis; RP, retinitis pigmentosum 19; XLSA/A X-linked sideroblastic anemia and ataxia. 
The clue to the function of $\mathrm{P}$-gp/MDR came from findings in bacterial periplasmic binding protein-dependent transport systems (early name for bacterial ABC). A conserved nucleotide-binding fold [37], containing Walker A and B, was found in hisP/malK/oppD, components of different transport systems, and oppD indeed bound to an ATP analog [38]. These findings led to a model that these transport systems were energy-dependent and ATP hydrolysis at hisP/malK/oppD were responsible for a conformational change to transmit the information to the substrate binding component [38]. By using the deduced amino acid sequence of P-gp/mdr, it was shown that $\mathrm{P}-\mathrm{gp} / m d r$ shared a high sequence homology with hisP/malK/oppD [39, 40] and hlyB [41] at the nucleotide-binding fold. Combined with the prediction from hydropathy plots, the proposed model of P-gp/MDR comprised of two highly conserved units, six putative membrane-spanning regions followed by a cytoplasmic nucleotide-binding fold, in tandem $[39,40]$. From the sequence homology with the bacterial transport systems, it was proposed that $\mathrm{P}-\mathrm{gp} / \mathrm{MDR}$ is an energy-dependent transporter that is involved in the efflux of drugs to reduce the drug accumulation in MDR cells [39-41]. By this time, it was realized that $\mathrm{ABC}$ proteins comprised a large protein superfamily [42] conserved from bacteria to mammals [43].

Forty-eight human $\mathrm{ABC}$ genes are classified into seven subfamilies (A to $\mathrm{G}$ ) based on the gene structure, amino acid alignment especially of nucleotide binding domains (NBD), and phylogenetic analysis and are named according to Human Genome Organization (HUGO) guidelines established in 1999 (P-gp/MDR1 is ABCB1 under the new nomenclature, http://www.genenames.org/genefamily/abc.php)[44]. ABC gene homologs found in other species, e.g., mouse, drosophila, are also classified into similar subfamilies. ABC proteins are characterized by the presence of Walker A and B motifs, which can be found in other ATP binding proteins, and an additional element, the signature motif, in NBD. Except for $\mathrm{ABCE}$ and $\mathrm{F}$ subfamilies, all $\mathrm{ABC}$ proteins also contain one or two sets of membrane spanning domains (MSD) consisting of six to eleven $\alpha$-helices. These MSD-containing ABC proteins will be referred to as ABC transporters hereafter although some of them, namely $\mathrm{ABCC}$, $\mathrm{ABCC} 8$, and $\mathrm{ABCC}$, are not typical transporters. $\mathrm{ABC}$ transporters can further be classified into two types by the number of functional units they contain. Functional transporters consist of two sets of MSDs and NBDs (Fig. 1.1.); full transporters contain two MSDs and NBDs in a single polypeptide, whereas half transporters contain only one MSD and NBD, thus are required to either homo- or heterodimerize in order to exert their functions. Full transporters are found in $\mathrm{ABCA}, \mathrm{ABCB}$, and $\mathrm{ABCC}$ subfamilies and half transporters are grouped into $\mathrm{ABCB}, \mathrm{ABCD}$ and $\mathrm{ABCG}$ subfamilies.

\subsection{ABC Transporters}

Although ABC transporters were initially identified from MDR cells and were implicated in MDR phenotypes, subsequent studies have shown that $\mathrm{ABC}$ transporters have more fundamental roles: moving molecules, whether xenotoxins or biological molecules, across the cell membranes in an energy-dependent and unidirectional manner. While most of the prokaryotic $\mathrm{ABC}$ proteins pump in compounds, mainly nutrients, from 
Figure 1.1. Schematic representations of $A B C$ transporters.

A. Full transporters contain two sets of MSDs and NBDs in tandem. In addition, long MRPs contain an extra MSD, MSD0. Domain structure of NBD is shown on the upper panel. B. Half transporters contain one set of MSD and NBD. ABCG proteins have a reversed orientation of MSD and NBD. Some ABCB half transporters contain an additional 3 - 5 TM helices. MSD, Membrane spanning domain; NBD, nucleotide binding domain; TM, transmembrane helices. 


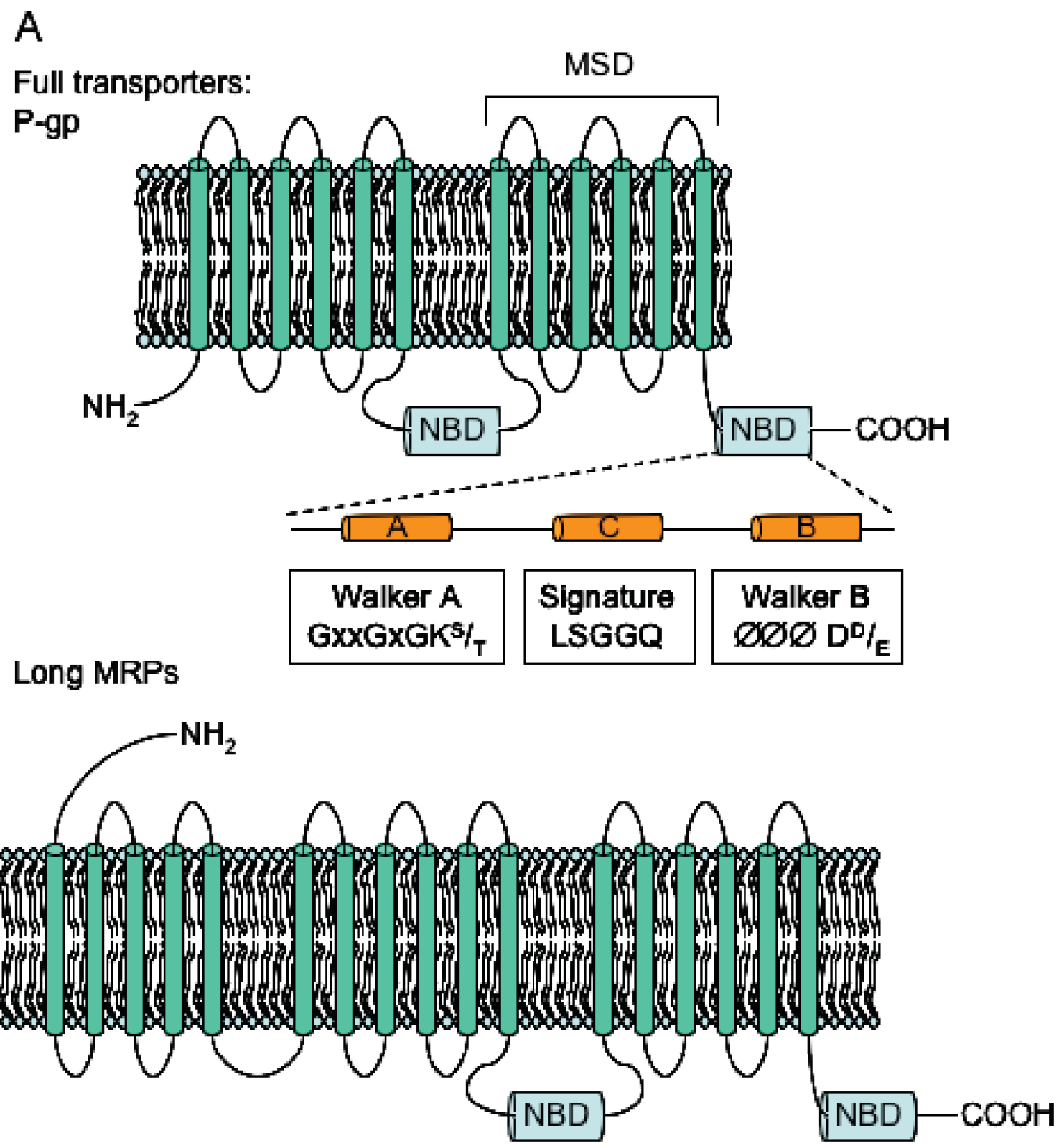



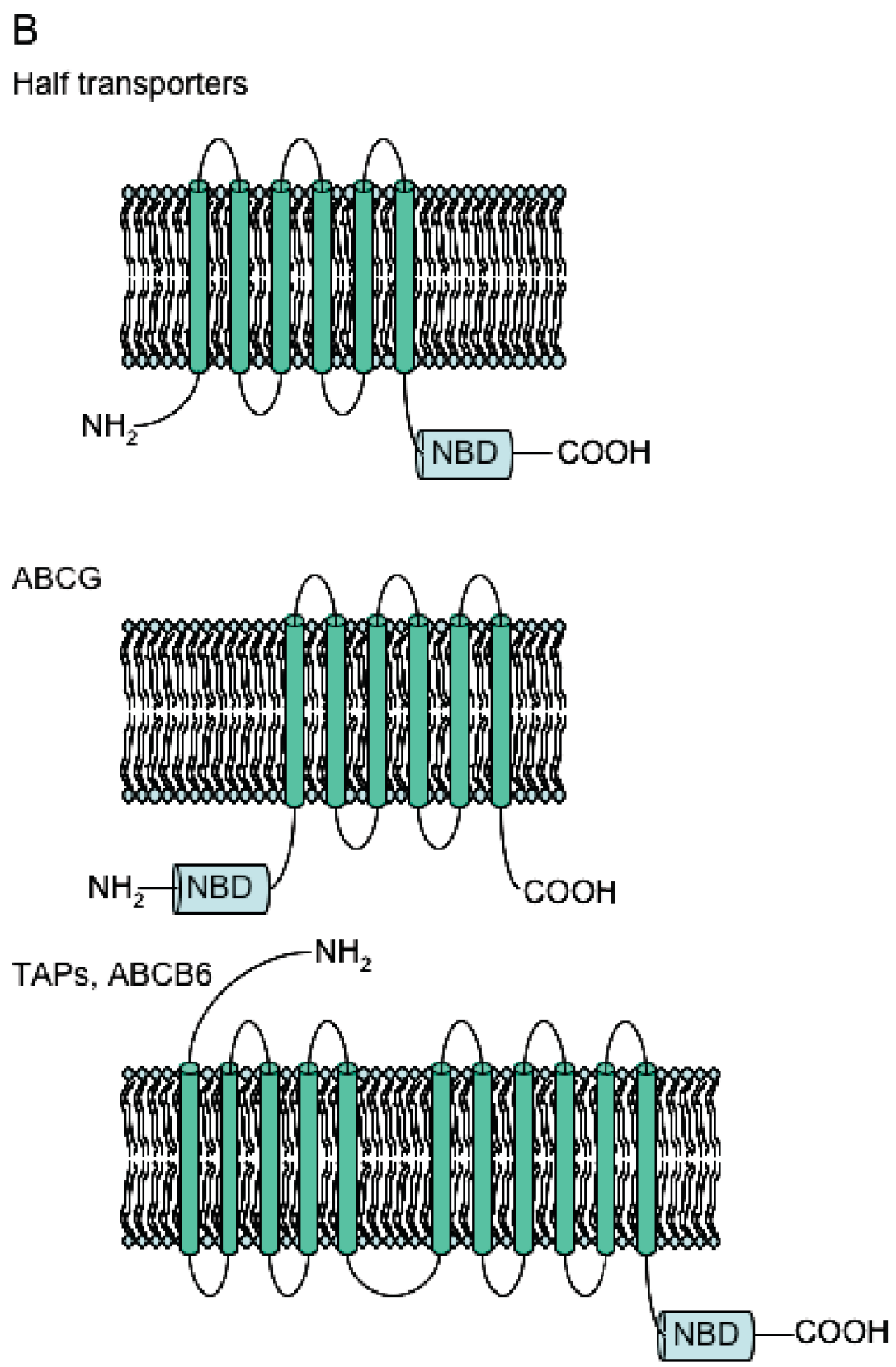

Figure 1.1. (continued). 
the extracellular milieu into the cells, eukaryotic $\mathrm{ABC}$ transporters typically move molecules from the cytoplasm to the extracellular compartment or into cellular organelles. Functions of some ABC transporters were identified through linkage analysis with known human disorders, tissue distribution patterns, and/or animal models.

\subsubsection{ABC Transporters and MDR Phenotypes}

Multidrug resistance frequently develops in patients undergoing chemotherapy and is one of the major reasons for the relapses observed. Mechanisms of MDR have been extensively studied for numerous agents and can be classified into either one or a combination of the following three patterns: i) decrease in the levels of uptake carriers for drugs that do not cross the membranes by passive diffusion, ii) mutations in the target molecules or target pathways so that the drugs are no longer effective, and iii) increase in levels of the transporters that efflux mainly hydrophobic drugs. Because many ABC transporters are able to efflux structurally different classes of drugs, changes in their expression levels can explain MDR phenotypes in many cases. Indeed, when MDR phenotypes developed in "spontaneous" mammary tumors from Brca1 $1^{-/ /} / \mathrm{p} 53^{-/-}$conditional mice in response to doxorubicin, $M d r l a$ and $M d r l b$ (mouse P-gp/MDR1) were consistantly upregulated in many of the tumors investigated [45]. The tumors also became cross-resistant to docetaxel, an excellent P-gp/MDR1 substrate, and became sensitive to doxorubicin when treated with a P-gp inhibitor suggesting that the upregulation of $M d r 1$ genes were responsible for the MDR phenotype [45].

Prior to 1991, P-gp was believed to be the only drug efflux transporter. However, "atypical" MDR phenotypes paved the way for identification of new ABC transporters that ply a role in MDR. For example, by taking an advantage of a doxorubicin-selected small cell lung cancer cell line, H69AR, with no P-gp overexpression, Cole et al., identified an ABC transporter, MRP1 (ABCC1) termed for multidrug resistanceassociated protein 1 [46]. In a concerted approach to identify P-gp independent resistance, Ross and colleagues utilized a cell line that was selected for doxorubicin resistance, but treated with the P-gp inhibitor, verapamil. From MCF7/AdrVp cell line, the third major drug transporter, breast cancer resistance protein (BCRP, also known as ABCG2), was identified [47]. When BCRP was stably overexpressed in MCF-7, the cells became resistant to doxorubicin, daunomycin, and mitoxantrone, meeting criteria as a multidrug transporter [47]. BCRP is also known as ABCP for its high placental expression [48] and as MXR for mitoxantrone resistance [49]. Currently, P-gp, MRP1, and BCRP are considered to be the three major "drug transporters" for their wide array of substrates and their roles in MDR phenotypes seen in many cell lines/tumors. Their substrates are not limited to chemotherapeutic agents as they can transport many other drugs including antiviral agents, antibiotics, and antimalarials (a concise list of their substrates is reviewed in ref. [50]).

Through the search of human expressed sequence tag (EST) database, the number of $\mathrm{ABC}$ proteins grew rapidly [44, 51]. Because of the role of P-gp and MRP1 as mutidrug transporters, other MDRs (ABCB full transporters) and MRPs have been 
extensively studied to identify clinically relevant substrates. Some were found to be overexpressed in drug resistant cells (such as MRP4)[51, 52] and others were found to confer drug resistance when ectopically overexrpressed in cells (for a list of drugresistant cell and transfection studies, see ref. [53]). There is a significant overlap in the substrates among transporters from different subfamilies [50, 54], but there are substrates that are characteristics of a certain subfamily. For example, many MRPs transport nucleoside and cyclic nucleoside analogs. ABC transporters can also transport drug metabolites such as glutathione or glucuronate conjugates or mono-, di-, or triphosphates.

Although not all drug transporters are upregulated in MDR cells, their ability to transport a wide array of compounds underscores the importance of identifying transporters for clinically used drugs. Not only do ABC transporters play a role in drug resistance, they also have critical roles in drug distribution and drug toxicity. For example, transporters may reduce drug efficacy by limiting the drug penetration into target tissues or impairing absorption leading to sub-therapeutic drug concentrations. On the other hand, reduced transporter function may increase susceptibility to adverse effects by increasing drug concentrations leading to prolonged exposure. Some ABC transporters have reduced activity secondary to non-synonymous single nucleotide polymorphisms (SNP). SNPs have been identified that affect the function of P-gp, MRP1, and BCRP (summarized in the ref. [55]). ABC transporters themselves can be drug targets given their roles in MDR, and several P-gp inhibitors are on clinical trials [53]. However, their physiological roles need to be determined to avoid any adverse effects.

\subsubsection{Physiological Roles of ABC Transporters}

In general, $\mathrm{ABC}$ transporters in the plasma membrane play an important role in pumping toxic compounds out of the cell or in maintaining cellular homeostasis, whereas transporters located on intracellular organelle membranes are involved in diverse biological or metabolic processes. Cell membranes provide a first line of barrier to limit the access of toxins into the cells or contain them in particular compartments within the cells. They can also act as a barrier to maintain a chemical gradient of compounds that do not readily cross the lipid bilayers. ABC transporters expressed on the cell surface add another layer of protection by actively pumping out toxins or metabolites. In accordance with their protective role, ABC transporters are expressed at such sites as blood-brain barrier (BBB), blood-placenta barrier, and luminal side of intestine, where the entry of toxins is strictly limited due to deleterious effects they may have. Development of animal models lacking $\mathrm{ABC}$ transporters has revealed unexpected but sometimes critical roles of these transporters in normal physiology.

Many compounds including drugs ingested orally are absorbed from the intestinal brush border membrane. Transporters such as P-gp/ABCB1, BCRP/ABCG2, and MRP2/ABCC2 are expressed on the apical membrane of intestine and limit the absorption of xenotoxins $[28,35,56]$. Increased oral availability of topotecan was

observed in BCRP knockout $(\mathrm{KO})$ mice supporting the barrier function of BCRP at the 
level of absorption [35]. Other transporters, such as obligate heterodimers of ABCG5 and ABCG8 that limit sterol absorption $[36,57]$, are also expressed in the intestine. Even if the compounds are absorbed in the intestine, they can still be metabolized by hepatic cytochrome P450 enzymes for detoxification and excretion through the bile and urine.

The bile mainly consists of cholesterol, various bile acids generated in hepatocytes from cholesterol, phospholipids, and bilirubin. Transporters located on the canaliculus membrane directly contribute to the content of bile, since they pump their substrates into the bile flow. Sinusoidal (basolateral) membranes of the hepatocytes come directly in contact with the bloodstream allowing the cells to take up whatever was absorbed at the intestine. They also provide a site for releasing the bile acid overload during cholestasis when bile flow is blocked or for releasing essential compounds generated in the hepatocytes, such as glutathione (GSH), to be circulated in the blood. As the major site for detoxification of endo- and exogenous compounds, liver contains a number of $\mathrm{ABC}$ transporters in order to move them across the membranes to other compartments. To name a few, canalicular transporters include P-gp/ABCB1 [56], BSEP/ABCB11 [58], MDR3/ABCB4 [59], MRP2/ABCC2 [28], and BCRP/ABCG2 [35], and sinusoidal transporters include MRP3/ABCC3 [60], MRP4/ABCC4 [61], and MRP6/ABCC6 [62]. Many of the substrates for the canalicular transporters were identified by analyzing bile contents from the knockout mouse models. For example, bile collected from $m d r 2^{--}$mouse (MDR3/ABCB4) was completely devoid of phospholipids highlighting the role of MDR3 as a biliary phospholipid transporter [59]. BSEP, as its name stands for bile salt export pump, transports hydrophobic bile acids into bile [58], which is then absorbed by intestine as part of the enterohepatic circulation. On the other hand, MRP2/ABCC2 pumps glucuronide-, glutathione-, or sulfate-conjugated compounds, which have become more hydrophilic than the original compound, into bile to be removed from the system [28]. ABCG5 and ABCG8 have been implicated in flipping the cholesterol from the inner to the outer leaflet of canalicular membranes affecting biliary excretion of cholesterol and other sterols in a MDR3-dependent manner $[63,64]$. Basolateral transporters MRP3 and MRP4 have been shown to transport bile acids, especially sulfated-bile acids, which cannot be handled by BSEP, into the blood perhaps to alleviate the toxic effects of hepatic bile acid accumulation during cholestasis $[60,61,65]$. The ABC transporters affect the fate of endo- and exogenous compounds both at the levels of intestinal absorption and the hepatic elimination. Mutations in many of these transporters are found to be the causes for human diseases. They are summarized in Table 1.1.

Another compartment protected by numerous transporters is brain. Because of its essential role in all animals, an entry of compounds into brain is limited by two barriers: $\mathrm{BBB}$ and blood-cerebrospinal fluid (CSF) barrier (BCB). The $\mathrm{BBB}$ function is maintained by the endothelium of brain capillaries; tight junctions between the cells do not allow paracellular movement of compounds from blood to the brain, whereas apical $\mathrm{ABC}$ transporters actively pump compounds into blood to limit their brain penetration. Several apical ABC transporters are reported including P-gp/ABCB1 [21], MRP2 [66], MRP4 [67], MRP5 [68], and BCRP [69]. Their role in BBB function was first identified in mdrla KO mice when the animals suffered neurotoxic side effects from ivermectin 
due to a 90-fold accumulation of the drug in the brain compared to the wild type mice [21]. Similar observations were made using Mrp2 deficient TR- rats and anti-epileptic drug [66], and Mrp4 KO mice and topotecan [67]. Apical localization of the transporters was also confirmed using immunohistochemistry. The second barrier in the brain to limit the uptake of the toxic compounds, $\mathrm{BCB}$, is maintained by epithelial cells in the choroid plexus. The apical surface comes directly in contact with CSF; therefore, the basolateral transporters pump compounds into blood to prevent brain penetration. MRP1 and MRP4 have been shown to localize on the basolateral membranes in choroid plexus and to contribute to the low etoposide [70] and topotecan [67] concentrations in CSF. P-gp localizes at the apical membrane, thus is not responsible for lowering the drug concentration at $\mathrm{BCB}$ [71]. Although the $\mathrm{ABC}$ transporters protect the brain from xenotoxins providing two layers of barrier at $\mathrm{BBB}$ and $\mathrm{BCB}$, they pose a major challenge to treating disorders in the central nervous system (CNS) including brain tumor. Therefore, it is critical to determine whether compounds are substrates for certain transporters in order to increase the efficacy without increasing systemic adverse effects. P-gp and BCRP inhibitors have been tested on pre-clinical models to increase brain distribution of CNS-targeted drugs.

Protective roles of $\mathrm{ABC}$ transporters extend to other compartments including materno-fetal interface where placental transporters prevent toxic agents from reaching the fetus. Significant accumulation of such drugs as paclitaxel and topotecan was found in $m d r 1 a^{-/} / 1 b^{-/}$and $\mathrm{Bcrp}^{-/}$embryos respectively $[35,72]$. Recent findings also implicate a protective role of $\mathrm{ABC}$ transporters in stem cells $[73,74]$. BCRP has been shown to be a stem cell marker and pumps Hoechst 33342 dye out contributing to the side population phenotype, which is used to isolate stem cells [74]. BCRP can also confer a survival advantage for stem cells under low oxygen conditions known as hypoxia by removing excess porphyrins and heme, which can damage membranes and other molecules [75].

All of the transporters described above that have the ability to pump out toxic compounds are expressed at the plasma membrane. Most of the half transporters belonging to $\mathrm{B}$ and $\mathrm{D}$ subfamilies reside on intracellular membranes and are involved in diverse biological processes. Transporters associated with antigen processing (TAP) 1 and $\mathrm{TAP} 2(\mathrm{ABCB} 2$ and $\mathrm{ABCB} 3$ respectively) localized on the endoplasmic reticulum (ER) are important molecules in immune response [76, 77]. After an antigen is degraded by proteasomes in the cytoplasm, TAP1/TAP2 heterodimers translocate the antigenic peptide across the ER membrane. The peptide is then loaded onto major histocompatibility complex (MHC) class I molecule, which can then present the antigen to $\mathrm{T}$ cells at the cell surface [78]. Mutations in TAPs are linked to immunodeficiency [23, 24]. TAP-like (TAPL, ABCB9) is expressed in the lysosomal membrane [79] where it is also thought to transport peptides [80]. Functions of other ABCB half transporters will be described in later sections. Although their substrates are not fully understood, peroxisomal transporters, $\mathrm{ABCD}$ subfamily members, are implicated in beta-oxidation of fatty acids [81].

Among the $\mathrm{ABC}$ transporters (ABC proteins with MSDs), cystic fibrosis transmembrane conductance regulator (CFTR, ABCC7), sulfonylurea receptor 1 (SUR1, 
ABCC8), and SUR2 (ABCC9) have unique roles in regulating ion concentrations. CFTR was initially identified as a cystic fibrosis $(\mathrm{CF})$ causing gene and was later found to function as a low conductance $\mathrm{Cl}^{-}$channel $[30,82]$. Three SUR proteins, SUR1, and two splice variants of SUR2, SUR2A and SUR2B, are regulators of ATP-sensitive $\mathrm{K}^{+}$ channels involved in such processes as regulating insulin secretion and vascular tone [83], and have no transport function identified to date.

\subsubsection{ABC Transporters and Diseases/Phenotypes}

About one third of known human $\mathrm{ABC}$ genes are associated with phenotypes linked to a genetic defect [2]. Well known genetic disorders associated with ABC genes include cystic fibrosis, retinal degeneration, and Dubin-Johnson syndrome (summarized in Table 1.1., [2]). Variations in a single nucleotide (creating a non-synonymous change) may be enough to produce deleterious effects. For example, a single nucleotide mutation in ABCB7, which has been implicated in iron homeostasis and iron-sulfur cluster formation, is linked to X-linked sideroblastic anemia and ataxia (XLSA/A) [26]. A wellcharacterized CFTR mutant, $\triangle \mathrm{F} 508$ results from a 3-bp deletion to produce a deletion of phenylalanine at position 508 [30]. This mutant cannot properly traffick to the plasma membrane and instead is targeted for degradation at the ER [84]. Lack of sufficient amount of CFTR, a chloride channel, at the membrane results in abnormal ion balance and fluid transport in airway cells, which ultimately leads to CF. Cystic fibrosis is the most common fatal genetic disease in Caucasians.

There are other SNPs or mutations reported in ABC genes that do not have severe clinical manifestations but may have effect on the protein function. For example, a mutation in P-gp gene will increase the sensitivity to drugs that are P-gp substrates. In a subset of dogs of the collie breed, a severely truncated P-gp is produced as a result of a 4bp deletion in exon 4. In these dogs, insufficient activity of P-gp at BBB causes sensitivity to ivermectin, a commonly used veterinary medicine for heartworm prevention $[21,22,85]$. A SNP in ABCC11 gene was found to be associated with earwax type, wet or dry [33]. Since ABCC11 is implicated in a cyclic nucleotide transport and has been shown to transport some drugs [86], it will be of an interest to see if the dry ear wax phenotype correlates with sensitivity to ABCC11 substrates. Phenotypes that can be seen immediately give an advantage over genotyping when considering tailor made therapeutics. Also, sensitivity to a particular drug may indicate impairment in some ABC transporters and, therefore, care should be taken when administering drugs that are substrates for such transporters.

\subsubsection{Architecture of ABC Transporters}

It is generally accepted that two sets of MSDs, each containing six transmembrane (TM) helices, and NBDs form a functional transport unit, with one set of MSD plus one NBD being considered a core unit (Fig. 1.1.)[87]. Introduction of a mutation in a single NBD is sufficient to abrogate transport activity, underscoring the 
importance of having two functional NBDs [88], even though ATP binding and hydrolysis at two NBDs are often not equivalent. When full transporters are separated into two core units ( $\mathrm{N}$ - and $\mathrm{C}$-terminal halves), co-expression of both halves is able to reconstitute transport activity, suggesting that functional transport units are not required to be in a single polypeptide $[89,90]$. In accordance, half transporters, which only contain a single core unit, either homodimerize as has been suggested for ABCG2 [91] or heterodimerize as seen in ABCG5/ABCG8 [57] and TAP1/TAP2 [92]. For obligate heterodimers, co-expression of two genes is essential for proper folding and maturation $[57,93]$. Higher oligomers are reported for some transporters, although their physiological roles remain elusive [91]. Domains that are important for dimer formation have been reported [94-96], but it is likely that more than one is responsible for the stable dimer formation. Recently reported structure of bacterial ABC multidrug transporter, Sav1866, also shows that TM helices from two MSDs intertwine to form two wings, which open up to release its substrates [97]. Crosslinking studies showed similar TM helices arrangement for P-gp [98].

MSDs have been implicated in substrate binding, thus share a low homology among transporters, whereas NBDs, which are responsible for binding and hydrolyzing ATP, are highly conserved [42]. As shown in Fig. 1.1., some MRPs contain an additional set of MSD, termed MSD0 with five TM helices, and some ABCB half transporters (i.e., TAPs) contain three to five additional TM helices [99]. While these domains may have an effect on basal transport activity [100], they have been shown to act as docking sites for interacting proteins [101] or regulatory domains for transport [102]. In ABCG subfamily, the arrangement of MSD and NBD is opposite from the other ABC transporters such that N-terminal NBD is followed by MSD. From biochemical and kinetic studies, it has been shown that $\mathrm{ABC}$ transporters are capable of binding multiple compounds simultaneously as seen in other multidrug binding proteins [103-107]. Although the use of UV-crosslinking of photoactivatable ligands and mutagenesis studies have also identified residues that are involved in the drug binding [108, 109], the structural evidence of substrate(s)-bound $\mathrm{ABC}$ transporters is required to fully understand the precise drug binding site(s). Based on structural information from bacterial multidrug binding protein QacR, the drug binding site is thought to contain a number of aromatic residues and a couple of charged amino acid residues to neutralize charged compounds $[105,106]$. Also, the drug binding site contains several "mini-pockets" that can accommodate different classes of drugs. These mini-pockets are continuous so that a bulky compound can utilize more than one mini-pocket for binding [105]. Since the substrate recognition is so complex, it is not feasible to predict substrate specificity based on the secondary structures.

To understand the function of NBDs, extensive mutagenesis studies have been performed on the conserved regions. In addition, soluble NBDs, unlike MSDs, are crystallized and their structures have been determined with or without nucleotide bound to them. NBD contains several characteristic domains including Walker A and B motifs, signature motif, switch region, and $\mathrm{A}, \mathrm{D}$, and $\mathrm{Q}$ loops each playing a critical role in nucleotide binding and/or hydrolysis (Fig. 1.2.). Both biochemical and structural studies have shown that two functional NBDs are required to form functional ATPases and that 


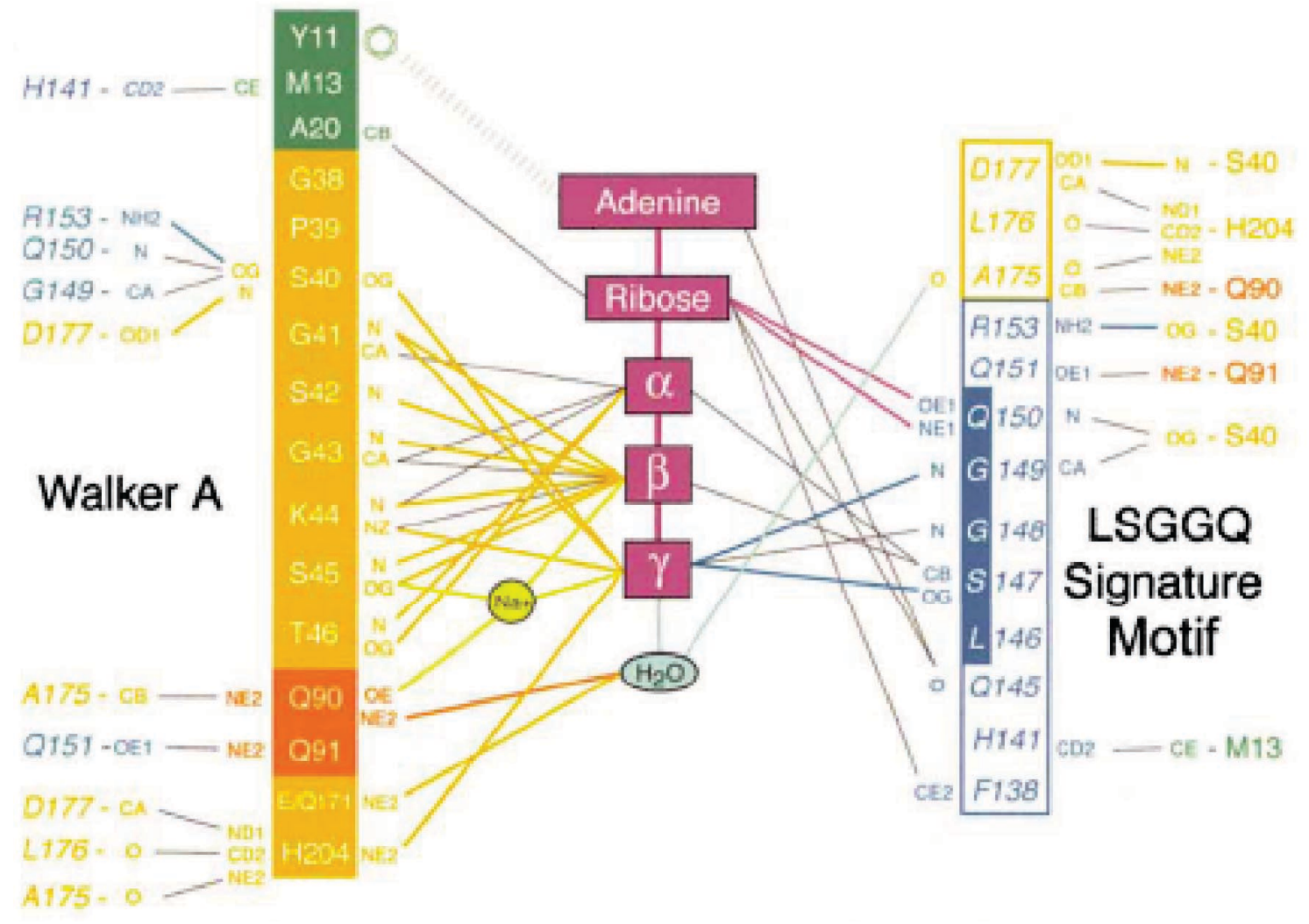

Figure 1.2. Interaction between ATP and different motifs from two NBDs. Mutagenesis studies as well structural data revealed interactions between an ATP molecule and key residues in different motifs. Motifs on opposite sides of ATP represent two NBDs. This diagram shows that an ATP molecule is sandwiched between two NBDs. This explains the need for two NBDs in functional ABC transporters. Reprinted from Molecular Cell, Vol 10(1), Smith, P.C., Karpowich, N., Millen, L., Moody, J.E., Rosen, J., Thomas, P.J., and Hunt, J.F., ATP binding to the motor domain from an ABC transporter drives formation of a nucleotide sandwich dimer, p. 139-149, Copyright (2002), with permission from Elsevier [110]. 
two molecules of ATP are bound at the dimer interface. The requirement for NBD dimer is readily understood by the observation that the Walker A and B motifs, switch region, A and Q loops from one NBD and the signature motif and D loop from the other NBD all contribute to make up the active ATPase [110]. The A-loop, an aromatic amino acid located $\sim 25$ amino acid residues upstream of Walker A motif, stacks the adenine ring from ATP, and the phosphates are stabilized by Walker A motif, whereas Walker B contains a catalytic glutamate. The histidine residue from the switch region promotes ATP hydrolysis by stabilizing the $\gamma$-phosphate and later the transition state $[110,111]$. Qloop binds to catalytic water, where D-loop (for dimerization) binds to $\mathrm{Mg}^{2+}$ as well as interacting with the catalytic water. The signature motif binds to $\gamma$-phosphate of ATP and plays a critical role in disengaging the dimer after ATP hydrolysis. However, an asymmetry in the NBD exists for many transporters. For TAP1/2, only one ATPase site constitutes of consensus motifs, where the other ATPase site has degenerate motifs implying that the consensus site is the primary site for ATP hydrolysis for substrate transport [111].

The Sav1866 structure as well the TAP1 structure revealed a soluble linker wrapped around the NBDs [97, 112]. In Sav1866, where the structure of the entire molecule was solved, the cytoplasmic loops were shown to interact with NBDs possibly transmitting information from NBDs to MSDs and vice versa [97]. Unexpectedly, the cytoplasmic loops were found to reach over to the NBD from the other molecule, making a twisted turn at the interface (Fig. 1.3.) [97].

\subsubsection{Transport Mechanism}

It has been shown that substrate translocation by $\mathrm{ABC}$ transporters requires ATP molecules. However, the precise mechanism of the translocation and the role of ATP binding and hydrolysis has been controversial. The initial model proposed by Senior et al. [113] included ATP binding to an empty NBD, which stimulates hydrolysis of the other ATP when a substrate is bound. This produces a change in affinity and results in the release of the substrate to the other side. ADP is released, which then resets the transporter to the high affinity state. The key points in this model are that only one ATP is hydrolyzed per transport cycle and that the ATP hydrolysis alternates between two NBDs. The other feature of this model is that the "power-stroke" which translocates substrates comes from ATP hydrolysis. On the contrary, recent structural and biochemical data favor the "ATP-switch" model, where ATP binding itself is the powerstroke [114]. In this model, substrate binding at high affinity site favors ATP binding to NBDs, which results in the formation of closed dimer. The change in the NBDs is then transmitted to MSDs inducing a conformational change to a low affinity state for substrates and the outward opening of the TM helices. The substrate is then released and the subsequent ATP hydrolysis followed by ADP and Pi release disengage the NBD dimer, resetting the transporter to a high affinity site. Accordingly, Sav1866 structure shows that the nucleotide binding stabilizes the outward opening of TM helices [97]. Substrate binding has been shown to induce a conformational change in many transporters [115] and relatively high $\mathrm{K}_{\mathrm{M}}$ for ATP in isolated NBDs may imply that the 

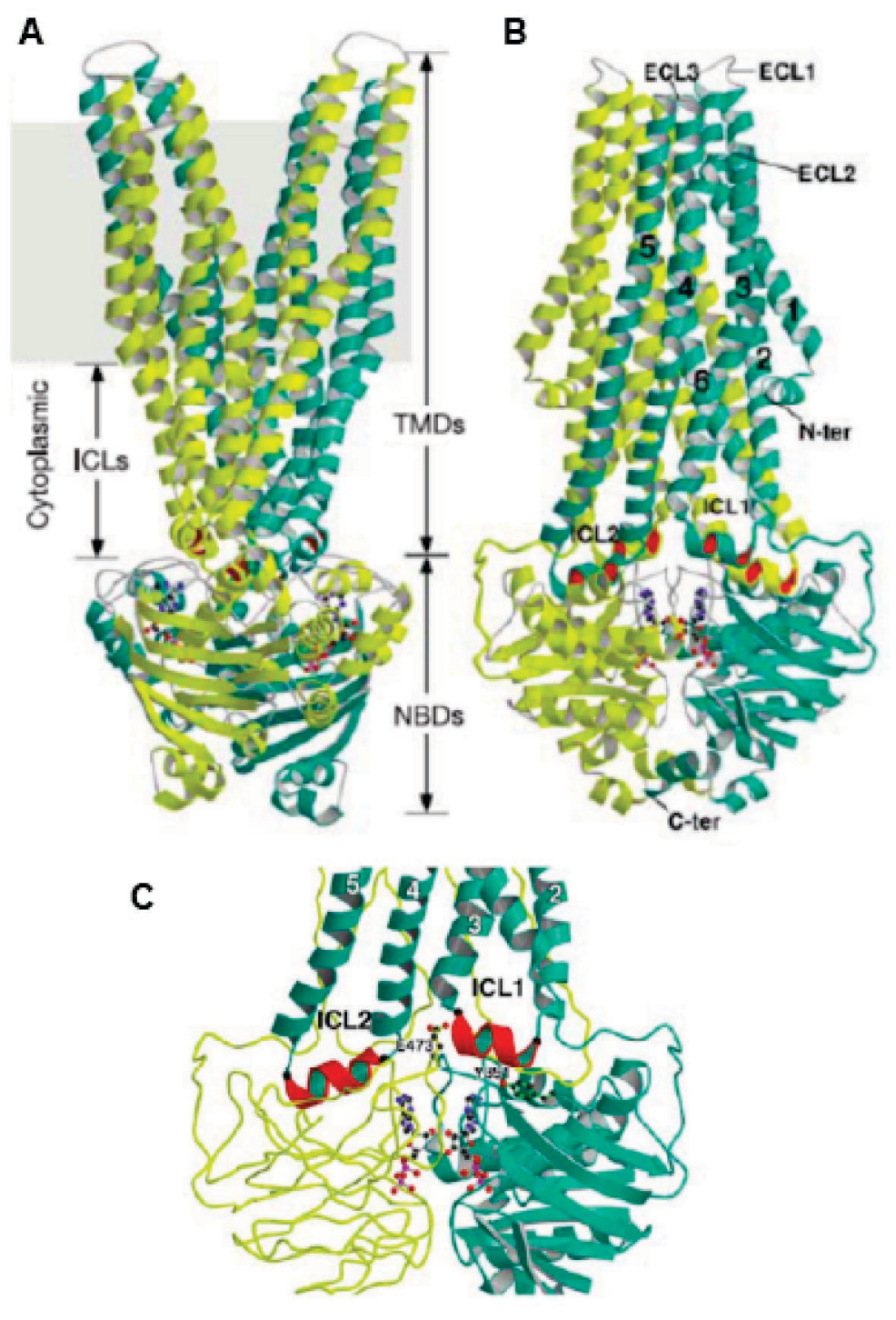

\section{Figure 1.3. Bacterial ABC transporter Sav1866 structure.}

Backbone of the homodimeric Sav1866 is shown in ribbon or coil representation with nucleotides bound at cytoplasmic NBDs. Yellow and turquoise represent each subunit in the dimer. A. This view shows membrane-embedded TM helices forming outward wings. TM helices from two subunits are intertwined to form the wings. Grey region indicates the position of predicted lipid bilayers. B. The protein is rotated $90^{\circ}$ around the vertical axis with resprect to A. TM helices in turquoise subunit are numbered. C. Close up view of the NBDs. This view shows the cytoplasmic loops coming from turquoise TM helices reach NBDs from subunits as colored in red. TM, transmembrane helices; NBDs, nucleotide binding domains; ICL, intracellular loops (between TM helices); N-ter, amino terminus; C-ter, carboxy terminus. Reprinted by permission from Macmillan Publishers Ltd: Nature (Dawson, R.J. and Locher, K.P., Structure of a bacterial multidrug ABC transporter. Nature, 2006. 443(7108): p. 180-185.), copyright (2006) [97]. 
changes in MSDs promote ATP binding.

\subsubsection{Regulation of ABC Transporters}

$\mathrm{ABC}$ transporter expression is regulated by transcription, translation, and degradation and their function is regulated by proper localization, post-translational modification, and/or interaction with other proteins. As xenobiotic pumps, many ABC transporters are known to be induced by their substrates and other xenobiotics that can activate nuclear receptors such as pregnane $X$ receptor (PXR), constitutive androstane receptor (CAR), farnesoid $X$ receptor (FXR), and peroxisome proliferators-activated receptor gamma (PPAR $\gamma)$ [116]. A number of bile acids activate FXR, which in turn induces BSEP/ABCB11, a bile acid transporter in the liver canaliculis $[117,118]$. PXR activators induce P-gp and MRP2, whereas CAR activators are known to induce P-gp, MRP2, and MRP4 [116]. These nuclear receptors regulate expression of many metabolizing enzymes namely cytochrome P450's, which can generate conjugated compounds that are substrates for the transpoters. CAR activation results in the coordinated upregulation of both Mrp4 and Sult2a1 [119]. Sult2a1 catalyzes the formation of sulfated steroids and bile acids, which can be removed from hepatocytes especially during cholestasis [119]. Because many drugs can induce or inhibit transporters, the drug-drug interaction may cause unexpected adverse effects. Stress stimuli are also known to induce $\mathrm{ABC}$ transporters in order to protect the cells. For example, BCRP/ABCG2 confers a survival advantage to cells during hypoxia and is induced by the binding of hypoxia-inducible factor 1 (HIF-1) to the hypoxia response element under hypoxic conditions [75]. Epigenetic changes such as promoter methylation and histone modification have also been shown to correlate with mRNA levels of transporters [120-123].

The usage of alternate promoters and splicing has been implicated in mRNA expression levels and tissue distribution of BCRP/ABCG2 [124-126]. In addition, alternative splicing has been reported for many of the ABCC subfamily members [127130]. Some alternatively spliced isoforms still produce functional proteins; however, some isoforms contain premature termination codons and are targeted for nonsensemediated mRNA decay $[129,130]$.

The expression of the $\mathrm{ABC}$ transporters can also be regulated at the translational level. A synonymous SNP in the coding region of P-gp has been shown to decrease the rate of translation resulting in a slightly different folding of the protein [131]. Incorrect

folding of the protein also does not leave ER and is targeted for degradation as seen with CFTR $\Delta$ F508 [84]. The mechanisms and the signals for sorting transporters to proper membranes such as apical and basolateral membrane, are also being extensively studied; however, much is unknown about signals that target transporters to correct membranes. Protein stability also plays a role in determining the levels of functional transporters. Post-translational modifications such as N-glycosylation of BSEP/ABCB11 and P-gp have been shown to affect protein stability or the proper trafficking $[132,133]$. Other $\mathrm{ABC}$ transporters are modified by glycan attachment. However, the role of N- 
glycosylation on $\mathrm{ABC}$ transporters has been somewhat inconclusive. For BCRP, the glycosylation has been shown not to affect its localization, expression, or transport activity [134].

Functional regulation of CFTR/ABCC7 has been extensively studied and the phosphorylation by PKA has been shown to activate its channel function. CFTR has also been shown to have several interacting partners through the C-terminal PDZ domain. Functional and physical coupling to the G protein coupled receptors (GPCR) such as $\beta_{2}$ adrenergic receptors [135] and type 2 lysophosphatidic acid receptor [136] have been reported. Since CFTR is activated by cyclic adenosine monophosphate (cAMP), the close proximity to a cAMP transporter, MRP4 through PDZ interaction attenuates its channel activity [137]. TAP1/2 also function in a macromolecular complex called peptide-loading complex, which consists of tapasin, MHC class I, ERp57, $\beta_{2}$-microglobulin, and calreticulin [138]. Further, interaction with other proteins may influence cell surface internalization and recycling as seen in GPCRs.

\subsection{Half Transporters in ABCB Subfamily}

The ABCB subfamily is unique among the other subfamilies because it contains both full and half transporters. There are six half transporters in this subfamily, ABCB2, $\mathrm{B} 3$, and $\mathrm{B} 6-\mathrm{B} 10 . \mathrm{ABCB} 2$ and $\mathrm{B} 3$ (TAPs) reside in the ER and ABCB9 (TAPL) in the lysosomes, and they are all involved in the translocation of peptides into the respective organelles. ABCB6-B8 and B10 localize at the mitochondria and are involved in other biological processes, although $\mathrm{ABCB} 10$ has been speculated to play a role in transporting peptides out of the mitochondria. Phylogenetic analysis revealed that within the B subfamily, there are three subgroups: the one containing full transporters, the second one containing ABCB2, B3, B8, B9 and B10, and the third one containing B6 and B7 [138]. It is not surprising that four transporters involved in peptide transport are clustered in one group even though their cellular localizations vary.

TAP1/2 are one of the well-characterized transporters beside the three major drug transporters. The TAP1 NBD has been crystallized and numerous mutagenesis studies showed mechanisms and kinetics of peptide transport cycle [111, 112, 139-144]. Moreover, the unique topology of TAPs with additional N-terminal TM helices was shown to be important for their interaction with tapasin, which is essential for forming the peptide-loading complex [99, 101]. TAPs translocate antigenic peptides into ER lumen to load them onto MHC class I molecules. Peptide-loaded MHC class I molecules are then dissociated from TAPs and present the antigen to T cells at the cell surface [138].

ABCB9 was initially identified from rat as a transporter homologous to TAP1 and TAP2 and was thus named TAP-like (TAPL) [145]. Mouse and human ABCB9 was later cloned and found to localize in lysosomes by immunofluorescence microscopy and gradient centrifugation [79]. Lysosomal localization of ABCB9 was confirmed by another group, which also showed ATP-dependent peptide transport into lysosomes by 
functional ABCB9 [146]. Unlike TAP1/2, ABCB9 is not involved in the antigen presentation by MHC class I and has been shown to homodimerize [80, 147]. Although the implication of its lysosomal localization is unclear, because ABCB9 expression is induced when monocytes differentiate into dendritic cells, professional antigen presenting cells, its role in delivering nuclear or cellular peptides to MHC class II has been suggested [146].

$\mathrm{ABCB} 8$ was the first mammalian mitochondrial $\mathrm{ABC}$ transporter to be characterized [148], but three other mammalian mitochondrial ABC transporters, $\mathrm{ABCB} 6, \mathrm{~B} 7$, and B10, have been reported to date. Immunofluorescence studies showed a co-localization of ABCB8 with MitoTracker, a mitochondria marker. Also, a faster mobility in SDS-polyacrylamide gel electrophoresis (SDS-PAGE) when expressed in intact cells compared to in vitro translated samples suggested a cleavage of a signal peptide. Indeed, the N-terminal of ABCB8 was sufficient for mitochondrial targeting of green fluorescent protein (GFP). Although the authors were not able to purify inner and mitochondrial outer membranes, the presence of a cleavable signal peptide led to a speculation that $\mathrm{ABCB} 8$ is in the mitochondrial inner membrane [148]. The substrates for ABCB8 are yet to be identified; however, it has been implicated in the cellular protection against oxidative stress [149]. Reduction of ABCB8 protein by small interfering RNA (siRNA) in neonatal rat cardiomyocytes leads to a loss of mitochondrial membrane potential. On the other hand, overexpression of ABCB8 protects cells from hydrogen peroxide-induced mitochondria dysfunction [149]. ABCB8 has been found in a large cross-linked complex containing four mitochondrial proteins although the physiological implication of the complex is unclear [150]. Regardless of what the substrates may be, $\mathrm{ABCB} 8$ is likely to be involved in the maintenance of mitochondrial membrane potential.

\subsection{1. $A B C B 7 / A t m 1 p$}

Saccharomyces cerevisiae Atm1p is the first mitochondrial ABC transporter to be identified and stands for $\underline{A B C}$ transporter of mitochondria [151]. Atm1p contains a functional mitochondrial targeting sequence at its $\mathrm{N}$-terminus and is localized at the inner membrane. The NBD of Atm1p has been suggested to reside in the matrix and, therefore, this transporter is considered to move its substrates into the intermembrane space. It forms a homodimer and a mutation in the conserved lysine residue in the Walker A domain disrupts dimer formation [94]. Yeast cells lacking atm 1 display slow growth with the increased oxidative stress considered to result from the mitochondrial free iron accumulation $[152,153]$. Increased sensitivity to the oxidative stress can be attributed partly to the reduced catalase activity. Although a decrease in the heme containing molecules is seen in the $\Delta$ atm 1 cells, including catalase and cytochomes, Atm1p is not considered a heme transporter $[151,152,154]$. In yeast cells where heme biosynthesis is defective, addition of heme rescues the slow growth phenotype, whereas heme does not affect the cells lacking atm 1, suggesting that reduced levels of heme is a secondary effect in $\Delta$ atm 1 cells [151]. On contrary, Atm1p has been shown to be involved in the generation of cytosolic [Fe-S] proteins [155]. Whether Atmlp directly transports the [Fe$\mathrm{S}$ ] cluster or not has not been shown. However, purified Atm1p reportedly shows ATPase 
activity stimulated by molecules containing sulfhydryl groups, especially by peptides containing cysteine residues [156]. If Atm 1p substrates include peptides containing sulfhydryl groups, then it is not surprising that another mitochondrial peptide transporter $m d l l$ was identified as a high copy suppressor of atm 1 to partially rescue the sensitivity to oxidative stress [157]. Mdl1p may be able to transport some of Atm1p substrate peptides in the absence of Atm1p. Regardless, it is clear that Atm1p plays a role in the iron homeostasis in $S$. cerevisiae. Recent findings in zebrafish show that [Fe-S] clusters are required for heme biosynthesis suggesting there is an intricate relationship between the two iron-containing molecules [158].

Mammalian ABCB7 was identified as the functional ortholog of yeast Atm1p [159], and mutations in this gene result in a partial loss of the function causing XLSA/A $[26,160,161]$. ABCB7 essentially has the same characteristics as the yeast Atm1p; it localizes to the mitochondria, contains a mitochondrial targeting sequence at the Nterminus, and restores growth, catalase activity, and normal iron level in $\Delta$ atm1 strain when overexpressed [159]. $A b c b 7$ null mice were embryonic lethal indicating that this gene is essential for development [162]. This explains the fact that only partial loss of protein function is observed in XLSA/A patients because fetuses harboring the mutations resulting in a complete loss of function will not be viable. This underscores the importance of [Fe-S] clusters as cofactors to many enzymes including respiratory chain, citric acid cycle, ribosome assembly, and amino acid synthesis [156]. Many of the tissuespecific conditional $A b c b 7 \mathrm{KO}$ mice were not viable with the exception of the liverspecific KO animals. Using the liver from these animals, the role of Abcb7 in the biogenesis of cytosolic [Fe-S] clusters was confirmed [162]. Total liver iron was increased in the knockout mice but there was no significant mitochondrial iron accumulation. Because cytosolic [Fe-S] clusters were unavailable, the cells responded as if they were under iron depletion (e.g., IRP binding to IRE was increased). Mitochondrion appears to be the site for regulating iron homeostasis; it is critical for [Fe$\mathrm{S}$ ] cluster formation, four of the eight steps in heme biosynthesis take place in the mitochondria, and mitochondrial ferritin is able to store free iron without damaging mitochondrial components. Therefore, it is not surprising that heme synthesis is altered in $A b c b 7 \mathrm{KO}$ animals or cells as a consequence to altered iron homeostasis especially in mitochondria. Increased ratio of zinc protoporphyrin IX (PPIX) to heme was observed in the blood from chimeric inducible- $A b c b 7 \mathrm{KO}$ animals [161] and increased PPIX was observed in the HeLa cells treated with ABCB7 siRNA [163]. These studies suggested that heme biosynthesis was not defective in these systems because PPIX is the penultimate product before forming heme and ferrochelatase catalyzes the insertion of both iron and zinc atoms into PPIX. Moreover, the mitochondrial ferritin-bound iron was significantly reduced in HeLa cells treated with ABCB7 siRNA even in the presence of mitochondrial iron accumulation, suggesting this pool of accumulated iron is inaccessible to ferrochelatase for heme biosynthesis [163]. Iron is an essential molecule to life and, therefore, its usage is tightly regulated. 


\subsection{2. $A B C B 10 / A B C-m e$}

ABCB10/ABC-me, named for its mitochondrial localization and erythroid expression, was originally identified as a GATA-1 target gene [164]. GATA-1 is a transcription factor essential for erythropoiesis, and it regulates many erythroid selective genes. ABCB10 expression in the sites of definitive hematopoiesis, such as fetal liver and adult bone marrow, also supported the potential role of ABCB10 in red cell development [164]. Dimethylsulfoxide (DMSO), a known inducer for erythroid maturation, increased ABCB10 mRNA expression in murine erythroleukemia (Mel) cells. Moreover, the rate of hemoglobin synthesis was greatly increased during DMSO-induced differentiation in Mel cells stably expressing ABCB10 [164]. However, the overexpression of ABCB10 in the absence of DMSO did not result in the spontaneous differentiation of Mel cells or increased hemoglobin synthesis; therefore, it is likely that ABCB10 facilitates the DMSO-induced cell differentiation and may require other regulatory proteins/pathways [164]. ABCB10 contains a mitochondrial targeting signal at its $\mathrm{N}$-terminus and localizes to the inner membrane with NBD in the matrix [164-166]. This orientation suggests ABCB10 pumps its substrates from matrix into the intermembrane space. ABCB10 has been shown to homodimerize and possibly form an oligomer [165]. The involvement of ABCB10 in hemoglobin biosynthesis and its mitochondrial localization suggested that ABCB10 might play a role in heme biosynthesis. As mentioned earlier, heme biosynthesis occurs both in the matrix and the cytoplasm. Therefore, although the substrates for ABCB10 are yet to be identified, it was suggested that ABCB10 transported heme or its precursors into the intermembrane space [164]. Heme, the end product in the heme biosynthesis, reduced ABCB10 mRNA suggesting a feedback mechanism to regulate heme biosynthesis [164]. However, the precise role of ABCB10 in erythropoiesis still needs to be determined.

From a phylogenetic analysis, yeast Mdl1p has been suggested to be an ABCB10 homolog [138]. They both localize to mitochondrial inner membranes and form homodimers $[165,167]$. Mdl1p exports peptides generated by m-AAA protease in the matrix into the intermembrane space [167]. Because ABCB10 clusters with other mammalian peptide transporters $\mathrm{ABCB} 2, \mathrm{~B} 3$, and $\mathrm{B} 9$, it may have a role in peptide transport; however, no direct evidence has been presented.

\subsubsection{Identification of ABCB6 (PRP/UMAT/MTABC3)}

As is the case with many $\mathrm{ABC}$ transporters, $\mathrm{ABCB} 6$ has more than one name. When a partial sequence of rat ABCB6 was cloned in 1997 as a gene upregulated during hepatocarcinogenesis, it was named P-gp-related protein (PRP) [168]. Full-length rat ABCB6 was cloned in 1998 and was identified as a half transporter [169]. Northern blot showed a ubiquitous expression of this transporter with testis having the highest expression level [169]. Both studies pointed out a high homology between rABCB6 and Schizosaccharomyces pombe heavy metal tolerance-1 (hmt1) protein. Hmt1 localizes to the vacuoles in yeast and confers a heavy metal resistance by sequestering toxic metals 
into vacuoles [170]. Whether $\mathrm{ABCB} 6$ has a functional homolog in yeast or not is yet to be determined.

In 2000, Mitsuhashi et al. cloned full length human ABCB6 (named MTABC3) and showed its mitochondrial localization using immunofluorescence microscopy and subcellular fractionation [171]. $A B C B 6$ gene is comprised of 19 exons and is located at chromosome 2q36. Ubiquitous expression of ABCB6 in many tissues including skeletal muscle, heart, brain, kidney, and pancreas, was confirmed. Because of its mitochondrial localization, ABCB6 was introduced into a yeast strain partially defective in Atm1p function to determine whether it can complement the function of Atm1p. Exogenous ABCB6 expression lowered amounts of free iron in mitochondria to a level comparable to Atm1p-expressed cells. However, neither ABCB6 nor Atm1p overexpression restored a normal level of mitochondrial iron. Moreover, a non-functional mutant of ABCB6 with mutations in the Walker A motif partially rescued $\triangle$ atm 1 phenotypes in these cells. These results suggested that the effect of $\mathrm{ABCB} 6$ expression on the iron level was unlikely to be transport-function dependent. Therefore, even though ABCB6 was suggested to be a homolog of Atm1p in this particular study [171], functional studies and the phylogenetic analysis clearly suggest that ABCB7, not ABCB6, is the true ortholog of Atm1p [159]. Taken together, ABCB6 appears to be involved in the mitochondrial iron homeostasis and possibly cell proliferation but its function remains largely unknown.

\subsubsection{Transcriptional Regulation of ABCB6}

Genome-wide analyses of transcription factor binding have led to a series of discoveries of previously unknown targets. In short, chromatin immunoprecipitation is performed using an antibody against a transcription factor of interest. Coimmunoprecipitated genomic DNA is analyzed by microarray to determine the identity of the sequences. ABCB6 promoter has been shown to potentially bind at least three transcription factor complexes from such studies. Li et al. showed that Myc/Max complex binds to the promoter of ABCB6 in Burkitt's lymphoma to possibly activate gene expression [172]. Similarly, p130 and E2F4 complex was found to bind to the promoter of ABCB6 under growth arrest condition to repress the expression [173]. Interestingly, they also identified nuclear respiratory factor-1 (NRF1) binding site in the promoter of ABCB6 [173]. NRF1 has been shown to regulate the expression of genes involved in mitochondrial function and biogenesis [174]. These findings suggest that ABCB6 is induced in rapidly proliferating cells and its expression may be coordinated with the expression of other mitochondrial proteins.

\subsection{Heme Biosynthesis}

Heme is an essential molecule and is, therefore, synthesized in almost all living cells with few exceptions. Organisms such as Caenorhabditis elegans, which does not have the capability of synthesizing heme, rely on exogenous heme for maintaining hemoproteins and iron homeostasis [175]. The tetrapyrrole structure, a backbone 
structure of heme and porphyrins, is seen in other molecules including chlorophyll and vitamin $\mathrm{B}_{12}$. Heme, an iron-containing PPIX, acts as a cofactor for numerous proteins involved in a variety of cellular processes. The production of heme is highest in the erythroid cells to sustain the robust hemoglobin synthesis, which is required for oxygen transport [176]. Another tissue with a high rate of heme biosynthesis is liver, which requires heme for the detoxifying enzyme cytochrome P450. In other tissues/cell types, heme is involved in many essential biological processes including respiratory chain, oxidative metabolism, and signal transduction [176]. Heme also regulates gene expression at transcription and translation level and affects protein stability and localization [176].

Even though heme is required for cellular functions, excess heme and porphyrins result in unfavorable outcomes [177]. First, iron in the heme can generate free hydroxyl radicals via Fenton's reaction, which damage cellular components such as DNA and membranes. Second, protoporphyrins are photosensitizers releasing singlet oxygen radicals in a light-dependent manner. Given the cytotoxic effects of iron, heme and porphyrins, cells tightly regulate heme biosyntheis by coordinating it with the synthesis of apo-hemoproteins. Additionally, removal of excess intracellular heme and porphyrins by cell surface transporters alleviates the cytotoxic effects. Heme catabolism by heme oxygenases into iron and biliverdin, which is ultimately converted to bilirubin, is another mechanism to eliminate excess heme.

\subsubsection{Heme Biosynthesis and Diseases}

The first step in heme biosynthesis takes place in the mitochondrial matrix, followed by four steps in the cytoplasm, and the subsequent three steps in the mitochondria complete the process (Fig. 1.4.)[176]. In humans, all eight enzymes involved in the heme biosynthesis have been cloned. The heme biosynthesis pathway is essentially the same in all human cells; however, because of the robust heme production in erythroid cells, erythroid-specific enzymes, either encoded by different genes or alternatively spliced, and different regulatory mechanisms exist in these cells. The first step in heme biosynthesis, the condensation of glycine and succinyl-CoA, is catalyzed by 5-aminolevulinate synthase (ALAS). In non-erythroid cells, the formation of ALA is the rate-limiting step. However, the translation of erythroid-specific ALAS2 protein is regulated by the availability of iron via the iron regulatory proteins/iron responsive element (IRP/IRE) pathway, which involves iron-sensing machinery through [Fe-S] clusters [179]. Therefore, in erythroid cells, the iron availability for heme is the ratelimiting step. The end product of the pathway, heme, can also inhibit ALAS2 by binding to the enzyme and retaining it in the cytoplasm. ALA formed in the mitochondrial matrix is then transported into the cytoplasm by a yet to be identified carrier(s), and is converted to a monopyrrole, porphobilinogen (PBG), by ALA dehydratase (ALAD). Subsequent enzymatic reactions by PBG-deaminase (PBGD), uroporphyrinogen III synthase (UROS), and uroporphyrinogen decarboxylase (UROD) form coproporphyrinogen III (CPgenIII). CPIII is then translocated into the intermembrane space of mitochondria, a step suggested to involve an energy-dependent carrier [180, 181]. CPgenIII oxidase in the 

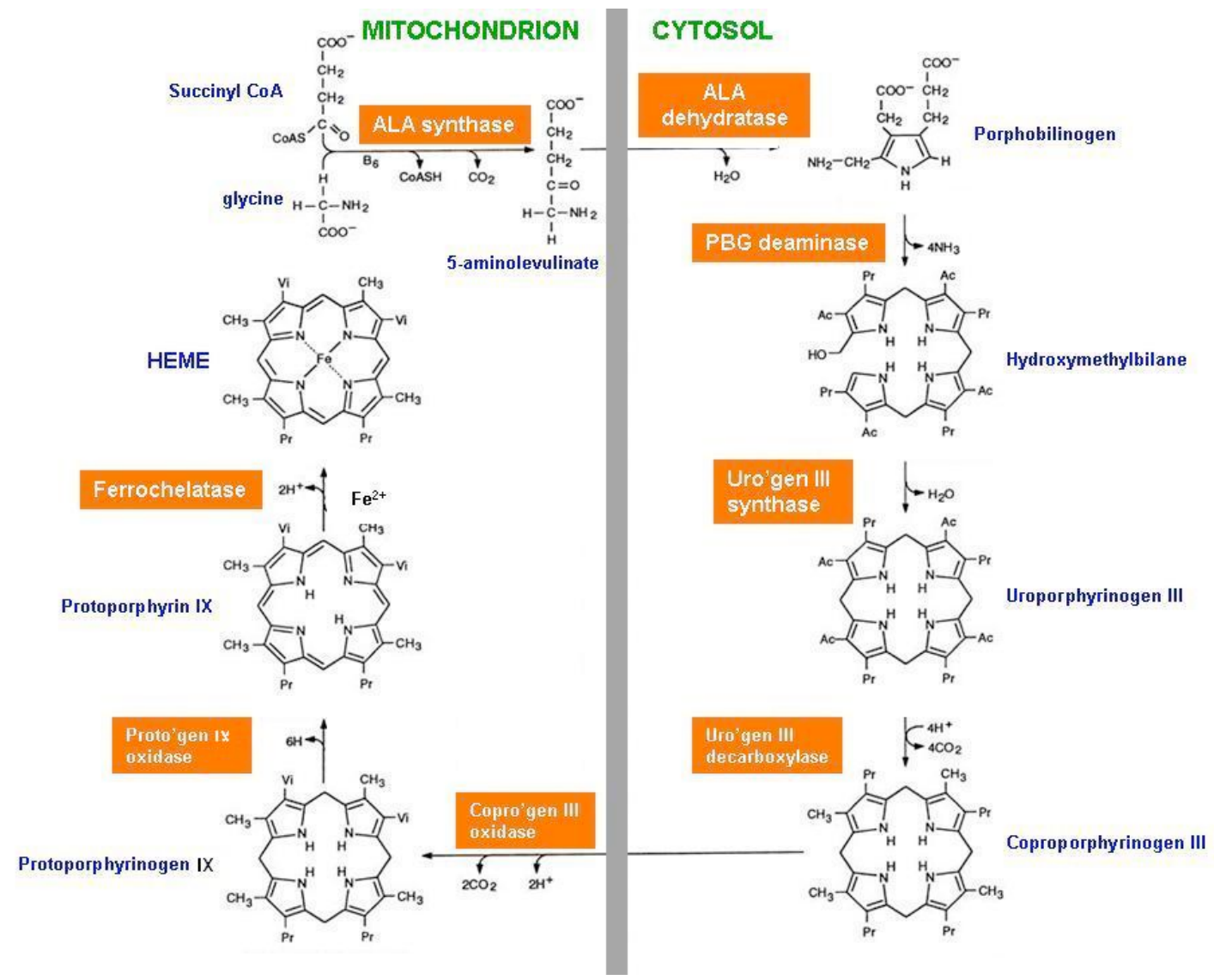

\section{Figure 1.4. Heme biosynthesis pathway.}

First step in heme biosynthesis occurs in mitochondria, followed by four steps in the cytosol. Coproporphyrinogen III oxidase and protoporphyrinogen IX oxidase are located in the mitochondrial intermembrane space, whereas the last step in heme biosynthesis, an insertion of an iron molecule into protoporphyrin IX, takes place in the matrix. Adapted by permission. http://www.reactome.org/cgi-bin/eventbrowser_st id?ST ID=REACT 9465.1, accessed September 14, 2008 [178]. 
intermembrane space then converts CPgenIII to protoporphyrinogen IX. The next enzyme in heme biosynthesis is protoporphyrinogen IX oxidase, an integral membrane protein in the mitochondrial inner membrane with the active site facing the intermembrane space. This enzyme generates the penultimate product, PPIX. The insertion of ferrous iron into PPIX is catalyzed by ferrochelatase, which is anchored to inner membrane with its active site facing the matrix. How PPIX translocates across the inner membrane is not fully understood. Mitochondrial iron used for heme is thought to be imported by mitoferrin in the inner membrane, a member of solute carrier family (SLC), specifically SLC25 subfamily [182]. At least three pools of iron have been suggested to exist in the mitochondria: iron stored in mitochondrial ferritin, [Fe-S] clusters, and a pool of iron readily available for ferrochelatase [163]. Further investigation is required to understand the precise mechanisms of iron trafficking and distribution in the mitochondria as well in the cytoplasm.

Every enzyme in the heme biosynthesis pathway has been shown to cause diseases when mutated, underscoring the importance of heme in mammals. Mutations in ALAS2 cause XLSA and mutations in the rest of the enzymes lead to various types of porphyria $[176,183]$. Clinical manifestations include photosensitivity, neuropathy, and acute porphyric attack but the severity of porphyrias varies among the subtypes. Also, sites of porphyrin overproduction and accumulation characterize two subgroups within the seven porphyrias. Defects in UROS and ferrochelatase lead to erythroid porphyrin overproduction, whereas defects in the other five enzymes result in hepatic porphyrias [183]. Although the porphyrias are inherited disorders, acute porphyria attacks can be induced by drugs and other chemicals.

\subsubsection{Compounds That Interfere with Heme Biosynthesis}

Chemically-induced porphyrias are the results of disturbance in the heme biosynthesis pathway. The most commonly used inhibitor for heme biosynthesis in the laboratory is succinylacetone (SA), which inhibits ALAD. However, there are several clinically relevant compounds that interfere with the heme biosynthesis pathway. Barbiturate drugs such as phenobarbital have been known to precipitate porphyria attacks in individuals with hereditary hepatic porphyrias by inducing ALAS1, which exaggerates porphyrin overproduction [184]. Heavy metals are also known to have effects on heme biosyntheis pathway and cause urinary porphyrin excretion in exposed individuals. Therefore, heavy metal contamination in the soil is a serious problem in some parts of the world. For example, lead toxicity amounts to clinical manifestations of porphyria attacks, because it binds to ALAD, and inhibit the enzyme [185]. Lead also inhibits the action of ferrochelatase by competing for the binding of iron. The ratio of zinc PPIX to heme increases under lead poisoning, which has been implicated in bone marrow toxicity [186]. Another heavy metal linked to porphyria is arsenite. Aresenite likely increases urinary porphyrin excretion by inhibiting PBGD and UROD [187]. Alcohol has also been linked to disruptions in heme biosynthesis, which may be due to an apparent pleiotropic inhibition of the following enzymes: ALAD, UROD, CPO, and ferrochelatase [188]. 


\subsubsection{Photodynamic Therapy}

The cytotoxic photosensitizing characteristics of porphyrins have proven to be quite useful when it comes to detecting and treating some tumors. Malignant tissues readily take up and accumulate porphyrins upon administration of ALA. In primary malignant glioma, accumulation of porphyrins produced a red fluorescence under specific wavelength (375-440 nm) to allow tumor visualization during surgery [189]. Fluorescing and non-fluorescing samples from the perimeter of the tumor were histologically determined to confirm that the porphyrin fluorescence was an excellent tumor marker. The ability of tumor to accumulate porphyrins is the cornerstone of photodynamic therapy [190]. Porphyrin-derivatives or ALA, are administered as photosensitizers, and activated by light at specific wavelength to induce cell death in tumor lesions. Underlying mechanisms of selective porphyrin accumulation and overproduction in tumor cells have not been fully understood. It is speculated that the rapidly proliferating tumor cells have a higher requirement for energy; thus, rate of heme biosynthesis is increased as with other cellular metabolisms.

\subsection{Heme and Porphyrin Transporters}

The requirement for heme and porphyrin transporters at least in the plasma membrane and the mitochondrial membranes is evident. Iron is essential to life and is absorbed at the intestinal membranes as inorganic iron or as the more bioavailable heme iron from myoglobin and hemoglobin. The uptake of heme at the cell surface has been suggested to be carrier-mediated [191]. On the other hand, heme biosynthesis occurs in mitochondria and cytoplasm, requiring movement of porphyrin precursors across mitochondrial membranes. This transmembrane movement has been suggested to involve energy-dependent processes but the identity of protein(s) responsible for such processes remains elusive. Moreover, exporters at the cell surface have been shown to play an important role in alleviating cytotoxic effects of heme and porphyrins under certain conditions. It is also possible that heme transporters on other organelle membranes exist in order to compartmentalize heme/porphyrins or to provide heme for apo-homoproteins.

Porphyrins and heme readily associate with cellular membranes due to their hydrophobic heterocyclic moieties, which may contribute to their damaging effects on membranes when activated. However, translocation from the outer leaflet of the lipid bilayer to the inner leaflet is a much slower process because of their anionic propionate side chains [192]. Therefore, the simple diffusion of heme precursor across the mitochondrial membranes is not sufficient to sustain the need for robust heme production in the erythroid cells. Accordingly, the presence of an energy-dependent transport of porphyrins into mitochondria has long been suggested [180,193]. Similarly, the activity of heme uptake at the plasma membrane has also been documented [191]. 


\subsubsection{Heme Importer: Heme Carrier Protein 1}

Heme carrier protein 1 (HCP1) was identified as heme importer from mouse duodenum where majority of heme absorption occurs [194]. HCP1 is a member of the major facilitator superfamily (MFS, SLC46A1) and localizes to the plasma membrane. Overexpression of HCP1 has been shown to increase heme uptake into cells [195]. In addition, HCP1 translocates from cytosol to the brush border membrane of enterocytes under iron-deprived conditions, suggesting that its function is regulated by cellular demand for iron to prevent possible iron overload [194]. Interestingly, Qiu et al. reported that HCP1 is a bona fide proton-coupled folate carrier by determining that mutation in this gene is the underlying basis for hereditary folate malabsorption [196]. Therefore, the physiological role of $\mathrm{HCP} 1$ on iron or heme homeostasis warrants further investigation.

\subsubsection{Heme/Porphyrin Exporters}

As mentioned in the earlier section, Bcrp is a porphyrin exporter at the cell surface [75]. Bcrp null mice showed photosensitivity due to an accumulation of photosensitizer pheophorbide a (PhA), a chlorophyll-metabolite, which has a tetrapyrrole backbone [35]. Not only does BCRP act as an exporter to remove exogenous porphyrins but it also acts to remove excess endogenous porphyrins [75]. Compounds with tetrapyrrole structures, but not a monopyrrole such as PBG, competed for specific binding of BCRP to hemin-agarose. These results suggested that tetrapyrrole backbone was important for substrate recognition [75]. Interestingly, BCRP also transports folate, illustrating the possible substrate overlap between HCP1 [197]. Due to activation of porphyrin biosynthesis, heme precursors are increased under hypoxic conditions, similar to levels of BCRP [75]. BCRP function is required to removes excess porphyrins under hypoxia protect cells from their cytotoxic effects.

Another heme exporter was identified through the search for feline leukemia virus, subgroup C (FeLV-C) cell surface receptor. FeLV-C receptor (FLVCR) was cloned and characterized as a member of MFS located at the cell surface [198]. Cats with FeLV$\mathrm{C}$ develop severe anemia due to red cell aplasia; therefore, Quigley et al. speculated that FLVCR might play a role in red cell differentiation. Accordingly, overexpression of FLVCR reduced heme accumulation into cells and the inhibition of FLVCR function by FeLV-C interfered with erythroid differentiation [199]. From these results, it was suggested that FLVCR acts as an "overflow valve" in erythroid cells to prevent the accumulation of free heme when there is not enough globin synthesized. Definitive proof was presented, however, when they showed that Flvcr null mice were embryonic lethal and lacked definitive erythropoiesis [200]. Flvcr null embryos had pale livers and lacked Ter119 positive cells, suggesting an arrest in erythropoiesis. The role of FLVCR as a heme exporter in differentiating erythroid cells emphasizes the importance of highly regulated heme biosynthesis and its utilization. Even in differentiating erythroid cells, where robust heme production is required, heme levels need regulation in order to avoid their cytotoxic and potentially deleterious effects on the terminal differentiation. However, FLVCR mRNA decreases as the erythroid cell differentiation proceeds [199], 
supporting the idea that FLVCR is required at the critical point where globin synthesis has not caught up with the heme biosynthesis.

In order to study the function of FLVCR, Flvcr ${ }^{\text {flox/flox;Mx-Cre }}$ was generated, where interferon administration induces a Cre recombinase activity. In this system, Flvcr gene can be knocked out in postnatal mice [200]. The mice developed severe hyperchromic macrocytic anemia with iron accumulation in such cells as splenic macrophages, hepatocytes, and duodenal enterocyes. Cardiomegaly and splenomegaly were also observed as consequences of severe anemia. These results indicate that FLVCR is involved in systemic iron homeostasis through heme transport. In human, FLVCR is highly expressed in tissues with high iron and/or heme traffick: duodenum, liver, spleen, uterus, placenta, and cultured macrophages. By using the iron-storage protein, ferritin, as a read-out, FLVCR was implicated in heme efflux from macrophages after they engulf senescent red blood cells. These results suggest that heme acquired from red blood cells may be recycled instead of being broken down by heme oxygenases. Also, Keel et al. speculate that the expression of FLVCR in the liver is important for biliary excretion of heme, although this claim needs further investigation.

\subsubsection{Intracellular Porphyrin Transporters}

Much is unknown about intracellular movement of heme and porphyrins. However, few proteins have been shown to either bind heme or be involved in the transport of heme or porphyrin. In many instances, porphyrin binding and transport are indistinguishable, making the interpretations of the data somewhat difficult.

Peripheral-type benzodiazepine receptor (PBR), originally identified as benzodiazepine receptor on the mitochondrial outer membrane, has been suggested to act as a porphyrin transporter. PPIX and CPIII were shown to compete for the binding of radiolabeled benzodiazepine to PBR [201]. Moreover, PBR expression was induced during the erythroid differentiation [202]. Taken together, Taketani et al. proposed a role for PBR in erythroid differentiation, acting as a porphyrin transporter in the heme biosynthesis pathway. There is no evidence to support this claim since direct porphyrin transport has not been shown to date. It is still possible that PBR is a porphyrin-binding component of a protein complex that trasnlocates porphyrins across the membrane.

Members of SLC25 subfamily are mitochondrial carrier proteins involved in the movement of many solutes and metabolites that are critical for mitochondrial functions. SLC25A11, also known as 2-oxoglutarate carrier (OGC), is a mitochondrial inner membrane protein, which transports 2-oxoglutarate into mitochondria in exchange for malate. Imported 2-oxoglutarate is then used for various metabolic pathways in the mitochondria including citrate cycle. Using metalloporphyrin-conjugated beads, OGC was identified as a porphyrin binding protein [203]. Hemin, PPIX, and CPIII all competed for 2-oxoglutarate uptake into mitochondria suggesting a competitive binding on OGC [203]. In addition, mitochondrial porphyrin uptake was inhibited by 2oxoglutarate [203]. These findings led to a conclusion that OGC is a porphyrin 
transporter involved in the movement of porphyrins from the intermembrane space to matrix. However, OGC inhibitor was only able to inhibit the porphyrin accumulation by $50 \%$ and the highest inhibition observed was about $40 \%$ at the highest dose of 2-

oxoglutarate used $(50 \mathrm{mM})$ [203]. The relevance of porphyrin transport by OGC in the heme biosynthesis pathway still needs to be determined. 


\section{CHAPTER 2. CHARACTERIZATION OF ABCB6 AS A PORPHYRIN TRANSPORTER ${ }^{*}$}

\subsection{Introduction}

Heme, an iron-containing porphyrin, is an essential molecule and has a crucial role in oxygen transport as a cofactor for globins [176]. However, heme plays important roles in many other biological processes such as metabolism and detoxification of biological compounds and xenotoxins (cytochromes, catalase, and peroxidase), oxidative phosphorylation (complex II-IV), signal transduction (adenylate and guanylate cyclases), and transcription regulation (N-PAS2 and Bach1) [176]. Heme also regulates a number of genes by affecting transcription, translation, protein targeting, and protein stability [205-209]. Even though heme and pophyrins are fundamental to life, excess heme and porphyrins have cytotoxic effects; iron in heme produces hydroxyl radicals via Fenton's reaction and porphyrins absorb light and generate oxygen radicals [177]. Therefore, intracellular levels of heme and porphyrin are tightly regulated. Deleterious effects of dysregulated heme biosyntheis are readily seen in the forms of various porphyrias or sideroblastic anemia $[176,183]$.

Heme biosynthesis takes place in two subcellular compartments: the mitochondria and the cytoplasm [176]. The first step in heme biosynthesis is the formation of ALA from glycine and succinyl-CoA in the mitochondrial matrix. The next step occurs in the cytoplasm where ALA is converted to a monopyrrole, PBG, followed by three enzymatic reactions to yield CPgenIII. The last three steps in heme biosynthesis take place in the mitochondria with an iron insertion into PPIX being the last step. The tetrapyrrole ring backbone, which can be found in heme and its precursors, chlorophyll and its metabolites, and vitamin B12, in CPgenIII is lipophilic and readily associates with the membrane. However, the anionic propionate side chains in CPgenIII make it a challenge to cross lipid bilayers with a simple diffusion [192]. In accordance, the uptake of the CPgenIII has been suggested to involve an energy-dependent active transport [193]. Even though several proteins have been identified to transport porphyrins across the plasma membrane (BCRP, FLVCR, and HCP1), the intracellular porphyrin transport process remains elusive [210]. However, the high rate of heme biosynthesis, especially in erythroid cells, requires such transporter(s) at the mitochondrial membrane to actively move heme precursor, CPgenIII, into the intermembrane space in order to facilitate the heme biosynthesis.

The $\mathrm{ABC}$ transporters translocate a wide array of compounds across the plasma membrane and the subcellular membranes in an ATP-dependent manner. Because of their ability to transport numerous compounds, $\mathrm{ABC}$ transporters play a role in many

\footnotetext{
* Modified with permission. Krishnamurthy, P.C., Du, G., Fukuda, Y., Sun, D., Sampath, J., Mercer, K.E., Wang, J., Sosa-Pineda, B., Murti, K.G., and Schuetz, J.D., Identification of a mammalian mitochondrial porphyrin transporter. Nature, 2006. 443(7111): p. 586589.
} 
biological processes including antigen presentation, insulin secretion, and cellular differentiation [2]. Physiological roles of many ABC transporters have been identified by the usage of $\mathrm{KO}$ animal models or by tissue distribution pattern. Variations in ABC transporters are the underlying molecular basis for a number of human disorders and phenotypes, which have also led to the identification of their function. The core unit of $\mathrm{ABC}$ transporters is composed of one MSD and one NBD aligned in tandem. Functional transporters require two of such units; MSDs composed of six TM helices bind substrates, whereas the NBDs bind and hydrolyze ATP molecules, thus, energizing the transport cycle. Full transporters contain two core units in a single polypeptide, whereas half transporters only contain one unit and are required to homo- or hetero-dimerize in order to exert their function. Half transporter ABCB6 localizes to the mitochondria [171], where three other members of the $\mathrm{ABCB}$ subfamily reside. $\mathrm{ABCB} 6$ is induced during liver regeneration and is associated with the mitochondrial iron homeostasis $[168,171]$. The mitochondria play an important role in cellular iron homeostasis because they are the sites for heme biosynthesis, [Fe-S] cluster formation, and iron storage in mitochondrial ferritin [156].

In order to understand the physiological role of $\mathrm{ABCB}$, other authors in this study examined the tissue distribution pattern of ABCB6. They demonstrated that ABCB6 mRNA is highly expressed in human fetal liver and mouse embryonic liver by Northern blot and in situ hybridization, respectively. In addition, database search revealed that $\mathrm{ABCB} 6$ is highly expressed in both human bone marrow and $\mathrm{CD} 71^{+}$early erythroid cells. These expression patterns suggested that ABCB6 is highly expressed in the site of erythropoiesis or in the cells with robust heme production. Increased cellular porphyrin levels by an addition of ALA or hemin, which is reduced to heme intracellularly, induced ABCB6 both at mRNA and protein levels. Taken together, we hypothesized that ABCB6 plays a role in intracellular trafficking of porphyrins. ABCB6 was also induced in the animal models with altered heme/porphyrin levels; increased splenic erythropoiesis by phenylhydrazine treatment or increased cellular PPIX levels due to a lack of Bcrp gene both induced $A b c b 6$ mRNA. These results further indicated that ABCB6 responds to an enhanced heme biosynthesis or increased cellular porphyrins. In order to gain insights into the role of ABCB6 in heme biosynthesis, the precise localization of ABCB6 in mitochondria was determined by separating mitochondrial inner and outer membranes on sucrose gradient. ABCB6 exclusively co-localized with the mitochondrial outer membrane markers. Further, proteinase $\mathrm{K}$ digestion on the intact mitochondria expressing $\mathrm{C}$-terminally tagged $\mathrm{ABCB} 6$ showed that the $\mathrm{C}$-terminus of $\mathrm{ABCB} 6$ localizes in the cytoplasm. These results indicated that $\mathrm{ABCB} 6$, localized to the mitochondrial outer membrane, transports its substrates into the intermembrane space from the cytoplasm. Given its location, direction of transport, and induction by elevated intracellular porphyrins, we hypothesized that ABCB6 may be the elusive porphyrin transporter that shuttles the heme precursor into the mitochondria. In accordance with this hypothesis, binding of $\mathrm{ABCB} 6$ to the agarose-conjugated hemin was confirmed.

My contribution to this study was to determine the substrates for ABCB6 and to show that the elevated levels of PPIX in ABCB6 overexpressing cells require functional $\mathrm{ABCB} 6$. By confirming that $\mathrm{ABCB} 6$ forms a homodimer, we were able to characterize 
ABCB6 function using cells overxpressing ABCB6 alone. The affinity for different heme precursors as well as other tetrapyrroles was tested to determine the structural requirement for $\mathrm{ABCB} 6$ binding. To definitively show that the transport function of ABCB6 was responsible for the observed effect of ABCB6 on heme biosynthesis, I generated human erythroleukemia cell line, K562, stably expressing either wild-type ABCB6 or non-functional Walker A ABCB6 mutant (in later chapter, this mutant is shown to be defective in ATP binding). Using these cell lines, other authors of this study showed that the ATP-dependent mitochondrial hemin uptake was seen only when the wild-type ABCB6 was expressed. I also showed that the substrate-stimulated ATPase and the elevated basal PPIX levels required a functional ABCB6. Other authors then showed that de novo heme biosynthesis was also upregulated in cells that expressed functional ABCB6. These findings indicate that ABCB6 is a porphyrin transporter that shuttles heme precursors into the mitochondria from the cytoplasm, thereby facilitating heme biosynthesis.

\subsection{Experimental Procedures}

\subsubsection{Cell Culture and Transfection}

NIH3T3 and K562 cells were cultured in Dulbecco's modified Eagle's medium (DMEM) containing $4500 \mathrm{mg} / \mathrm{L}$ glucose supplemented with 10\% FCS, $2 \mathrm{mM} \mathrm{L-}$ glutamine, and penicillin and streptomycin (Gibco) in the presence of 5\% $\mathrm{CO}_{2}$ and humidified atmosphere. Cells were transfected using LipofectAMINE Plus (Invitrogen)

according to the manufacturer's protocols. Cells were plated on 6 well-clustered dish at 3 $\mathrm{x} 10^{5}$ cells/well 24 hours prior to transfection. For each transfection, $1 \mu \mathrm{g}$ of indicated plasmid was used unless otherwise noted.

To obtain K562 cells stably expressing ABCB6-V5 or ABCB6-K629G-V5, pcDNA3.1-hABCB6-V5 or pcDNA3.1-hABCB6-K629G-V5 was introduced into K562 cells by electroporation. Cells were selected with G418 for two weeks before the expression of ABCB6 was tested by immunoblotting.

\subsubsection{Site-Directed Mutagenesis}

Plasmids pcDNA3-hABCB6-FLAG and pcDNA3.1-hABCB6-V5-His were designed to express human ABCB6 with C-terminal tags, FLAG or V5 respectively. A point mutation was introduced into pcDNA3.1-hABCB6-V5-His using a Quikchange site-directed mutagenesis kit (Stratagene, La Jolla, CA) and primers (K629G; sense, 5'GTGGGCCCATCTGGGGCAGGGGGGAGCACAATTTTGCGCCTGCTG-3', antisense, 5'-CAGCAGGCGCAAAATTGTGCTCCCCCCTGCCCCAGATGGGCCCAC-3', G508L; sense 5' - CAGAACCTGGTGATTGGGCTCCTGCTCCTCGCCGGCTCCCTGCTTTG-3', antisense, 5'- CAAAGCAGGGAGCCGGCGAGGAGCAGGAGCCCAATCACCAGGTTCTG-3', G512L; sense 5'- GATTGGGCTCGGGCTCCTCGCCC- 
TCTCCCTGCTTTGCGCATACTTTG-3', antisense, 5'- CAAAGTATGCGCAAAGCAGGGAGAGGGCGAGGAGCCCGAGCCCAATC-3', G508,512L; sense, 5'- GAACCTGGTGATTGGGCTCCTGCTCCTCGCCCTCTCCCTGCTTTGCGCATAC-3', antisense, 5'- GTATGCGCAAAGCAGGGAGAGGGCGAGGAGCAGGAGCCCAATCACCAGGTTC-3', D641A; sense, 5'- GCCTGCTGTTTCGCTTCTACGCCATCAGCTCTGGCTGCATCCG-3', antisense, 5'- CGGATGCAGCCAGAGCTGATGGCGTAGAAGCGAAACAGCAGGC-3', D650A; sense, 5'- GCTCTGGCTGCATCCGAATAGCTGGGCAGGACATTTCACAGGTGAC-3', antisense, 5'- GTCACCTGTGAAATGTCCTGCCCAGCTATTCGGATGCAGCCAGAGC-3'). All genes were thoroughly sequenced after mutagenesis.

\subsubsection{Immunoblotting and Immunoprecipitation}

Twenty-four hours after transfection, cells were washed with phosphate buffered saline (PBS) and harvested into $1 \mathrm{ml}$ ice-cold PBS containing 1x protease inhibitor cocktail (Complete EDTA free, Roche). Cell suspensions were centrifuged at 1,500 $\mathrm{x}$ $\mathrm{rpm}$ for $4 \mathrm{~min}$ at $4^{\circ} \mathrm{C}$. Cells were resuspended in Buffer A ( $50 \mathrm{mM}$ Tris-HCl (pH 7.4), $150 \mathrm{mM} \mathrm{NaCl}, 10 \%$ glycerol, and 1 x protease inhibitor cocktail (Roche)), and then sonicated. After the final concentration of $1 \% \mathrm{NP}-40$ was added, the cells were solubilized for $60 \mathrm{~min}$ at $4^{\circ} \mathrm{C}$ with a constant rocking, followed by centrifugation at 3,000 $\mathrm{x} \mathrm{rpm}$ for $5 \mathrm{~min}$ at $4^{\circ} \mathrm{C}$ to remove cell debris. The supernatant was further subjected to immnoblotting or immunoprecipitaiton after protein concentration was measured by Bradford method (Protein Assay, Bio-Rad Laboratories, Inc.). Final concentration of $1 \mathrm{x}$ Laemmli sample buffer (Bio-Rad) was added to the supernatant for immunoblotting. For immunoprecipitation, the lysate was incubated with an anti-V5 monoclonal antibody (Invitrogen) or an anti-FLAG antibody monoclonal antibody (M2, Sigma) at 1:150 dilution, and Protein A Sepharose beads (Amersham Pharmacia Biotech., Liscataway, NJ) for $2 \mathrm{hr}$. The samples were washed twice with wash buffer (Buffer A containing 1\% NP-40), and then Laemmli sample buffer was added to the immnoprecipitates. For immnoblotting, proteins were separated on 10\% SDS-PAGE and transferred onto nitrocellulose membrane (Schleicher \& Schuell Biosciences, Inc., Keene, NH). The membrane was blocked with $10 \%$ skim milk in PBS-T (PBS and $0.1 \%$ Tween 20 ) and then incubated with an anti-V5 polyclonal antibody (MBL, Japan) or anti-FLAG polyclonal antibody (Sigma). The blot was washed with PBS-T, and then incubated with horseradish peroxidase-conjugated anti-rabbit IgG F(ab') $)_{2}$ fragment (Amersham Pharmacia Biotech.). The blot was washed again with PBS-T and detection was performed with enhanced chemiluminescence detection (ECL, Amersham Pharmacia Biotech.).

\subsubsection{Crude Mitochondria Preparation}

K562 cells were pelleted, washed with PBS, and resuspended in resuspension buffer $(10 \mathrm{mM} \mathrm{NaCl}, 1.5 \mathrm{mM} \mathrm{MgCl} 2,10 \mathrm{mM}$ Tris- $\mathrm{HCl}(\mathrm{pH}$ 7.6), and $1 \mathrm{x}$ protease inhibitor cocktail (Roche). After 10 min incubation on ice to allow the cells to swell, they 
were broken with type B Dounce homogenizer. $2.5 \mathrm{x}$ membrane stabilizing buffer (525 $\mathrm{mM}$ mannitol, $175 \mathrm{mM}$ sucrose, $12.5 \mathrm{mM}$ Tris-HCl (pH 7.6), and 2.5 mM EDTA) was added to the homogenate to a final concentration of $1 \mathrm{x}$. The samples were centrifuged at $1,300 \mathrm{x} \mathrm{g}$ for $5 \mathrm{~min}$ at $4{ }^{\circ} \mathrm{C}$ to remove cell debris and nuclei. The supernatant was further centrifuged at $17,000 \mathrm{xg}$ for $15 \mathrm{~min}$ at $4^{\circ} \mathrm{C}$ to pellet mitochondria.

\subsubsection{Hemin-Agarose Pull-Down Assay}

Cell lysates prepared from NIH3T3 cells transiently expressing ABCB6-V5 or ABCB6-K629G-V5 as described above were incubated with hemin-agarose for $30 \mathrm{~min}$ at $24^{\circ} \mathrm{C}$. For competition assays, indicated compounds were preincubated with the lysates for 5 min prior to an addition of hemin-agarose. The resin was washed for three times with wash buffer, and the proteins were eluted using $1 \times$ Laemmli sample buffer containing $\beta$-ME. ABCB6 bound to hemin-agarose was analyzed by immunoblotting.

\subsubsection{ATPase Activity Measurement}

The vanadate-sensitive ATPase activity in the crude mitochondria isolated from K562, K562-ABCB6-Flag, K562-ABCB6-V5, and K562-ABCB6-K629G-V5 (Walker A mutant) was measured by the colorimetric assay as described previously [211]. The isolated mitochondria preparations containing $30 \mu \mathrm{g}$ of protein were incubated with indicated concentrations of CPIII (prepared in methanol) or PBG (prepared in $\mathrm{ddH}_{2} \mathrm{O}$ ), and the reaction was started with the addition of $3.3 \mathrm{mM} \mathrm{MgATP}$. The vanadate-sensitive CPIII- and PBG-stimulated ATPase activities were detected and calculated as the release of inorganic phosphate (nmol Pi released $/ \mathrm{min} / \mathrm{mg}$ protein). Then the ATPase activity was expressed as a fold change relative to the basal vanadate-sensitive ATPase activity. The values are average of two to four measurements \pm S.E.M.

\subsubsection{Cellular PPIX Measurements}

Cellular PPIX levels were measured by flow cytometry. Cells were harvested by centrifugation, washed and resuspended in PBS. The PPIX fluorescence was measured using a Vantage flow cytometer (BD Biosciences) in which the excitation wavelength was set at $405 \mathrm{~nm}$ and the emission filter was set at 695/40 nm.

\subsection{Results}

\subsubsection{ABCB6 Homodimerizes}

Half transporters need to either homo- or hetero-dimerize in order to exert their functions; however, whether ABCB6 homodimerized had not been determined. We 
postulated that $\mathrm{ABCB} 6$ functions as a homodimer because of two findings: i) overexpression of ABCB6 alone in cells elevated PPIX levels and ii) three other known mitochondrial $\mathrm{ABC}$ transporters localize in the inner mitochondrial membrane and, therefore, are unlikely to dimerize with the mitochondrial outer membrane ABCB6. To test if $\mathrm{ABCB} 6$ homodimerized, co-immunoprecipitation using differently tagged $\mathrm{ABCB} 6$ was performed. ABCB6-Flag and ABCB6-V5 were transiently co-expressed in NIH3T3 cells and lysates prepared. Anti-V5 or anti-Flag antibody was used to immunoprecipitate V5- or Flag-tagged ABCB6, respectively, and Flag- or V5-tagged ABCB6 in the immunocomplex was analyzed by immunoblotting (Fig. 2.1.). Both V5- and Flag-tagged ABCB6 were found in the immunoprecipitates when anti-Flag antibody was used to immunoprecipitate $\mathrm{ABCB} 6-\mathrm{Flag}$ indicating that $\mathrm{ABCB} 6-\mathrm{V} 5$ and $\mathrm{ABCB} 6-\mathrm{Flag}$ interact. Similar results were obtained when anti-V5 antibody was used to immunoprecipitate ABCB6-V5. These results indicate that ABCB6 homodimerizes and suggest that it does not require a heterodimeric partner.

To determine the function of $\mathrm{ABCB} 6$, we then developed mutant $\mathrm{ABCB} 6$ and focused on a domain that has been reported to affect either dimerization and/or function. Substitution of the conserved lysine residue in the Walker A motif in the NBD impairs transport activity of many $\mathrm{ABC}$ transporters [212, 213]. Further, previous studies have shown several consequences of the substitution of this residue in half-transporters including the loss of stable homodimer formation of Atm1 $\mathrm{p}$ [94], the decreased targeting of BCRP to the plasma membrane [214], and the loss of ATP binding by TAP1 [212]. We generated a mutant ABCB6 construct in which the conserved lysine residue is substituted with a glycine residue. Differently tagged wild-type and Walker A mutant ABCB6-K629G were transiently transfected in NIH3T3 cells in the indicated combinations (Fig. 2.2.). Co-immunoprecipitation using anti-Flag antibody showed that the Walker A mutant both homodimerizes and heterodimerizes with wild-type ABCB6.

ABCB6 contains two other motifs that have been shown to affect the packing of $\alpha$-helices of membrane proteins or homodimerization of yeast protein. Therefore, we next tested the contribution of these two motifs in ABCB6 dimerization. In several membrane proteins, the GXXXG motif is suggested to be important in the packing of $\alpha$-helices in transmembrane regions [215-217]. Based upon studies showing a conserved GXXXG was important for BCRP transport activity [95], we also evaluated the contribution of this motif to ABCB6 homodimerization. There are four GXXXG motifs present in ABCB6; therefore, in order to select GXXXG motif that may reside in the membrane-spanning domain, transmembrane $\alpha$-helix regions in ABCB6 were predicted using three programs, SOSUI, TopPred, and TMHMM [218-220]. All three programs predicted only the GXXXG at amino acid residues 508 through 512 to be in an $\alpha$-helix. Thus, point mutations were introduced to substitute Gly508 and Gly512 with leucine residues. Yeast protein Gts1p homodimerizes through a region (DXXXGXXXXD) that is very similar to a downstream region of the Walker A motif of several $\mathrm{ABC}$ transporters [221]. Also, the binding of C-terminal regions of yeast $\mathrm{ABC}$ transporter Mdl1p to Gtslp was lost or diminished when the aspartate residues were substituted with alanine residues [221]. ABCB6 contains a highly similar sequence at the downstream of the Walker A motif. Thus, Asp641 and Asp650 were substituted with alanine residues to evaluate the role of 

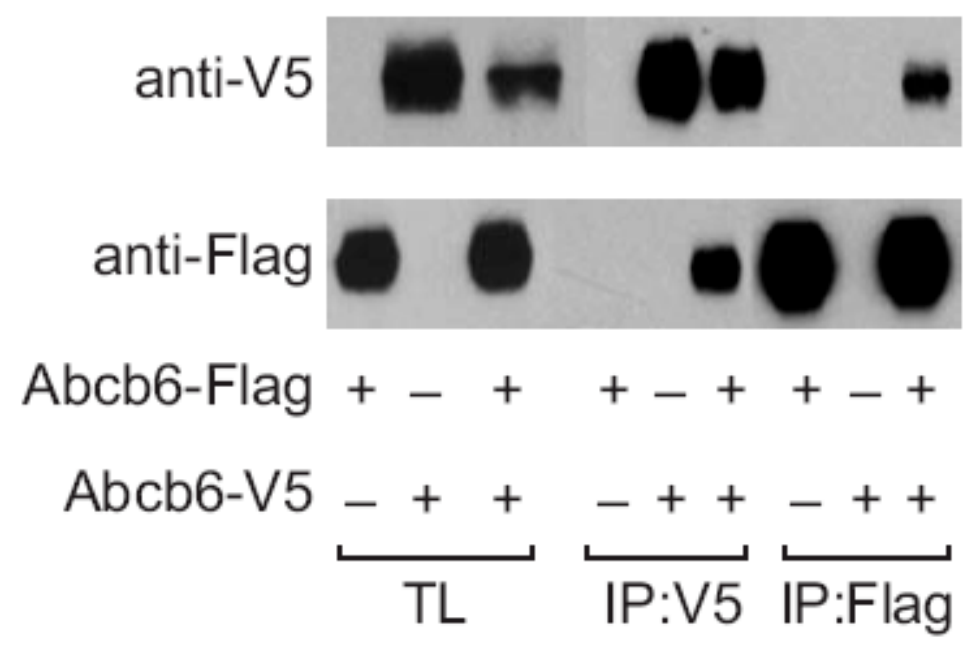

Figure 2.1. Homodimerization of ABCB6.

NIH 3 T3 cells were transiently transfected with plasmids encoding ABCB6-FLAG and ABCB6-V5. Twenty four hours after transfection, cell lysates were prepared and were incubated with anti-FLAG (M2) or anti-V5 antibodies and with Protein A Sepharose beads. The immunoprecipitates were washed twice with wash buffer, and the samples were analyzed by immunoblotting. TL: total lysate. 


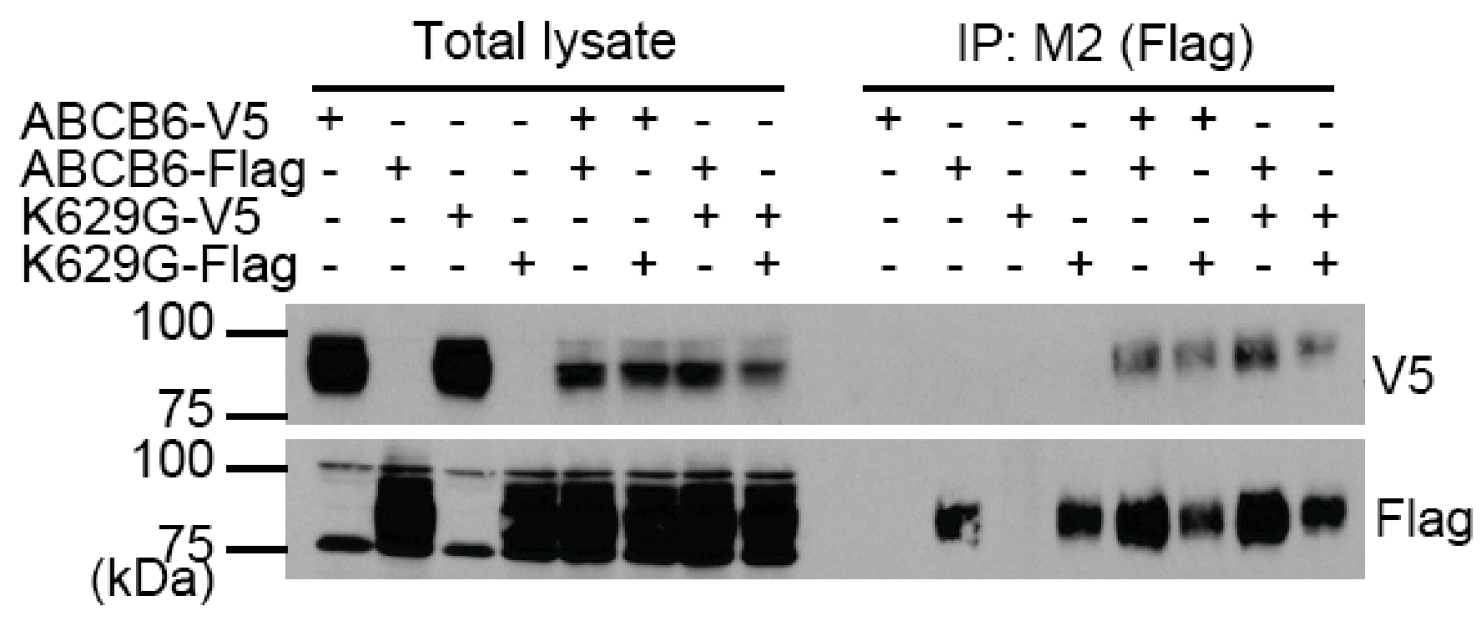

Figure 2.2. Mutation in the Walker A motif does not affect ABCB6 dimerization. NIH $3 \mathrm{~T} 3$ cells were transiently transfected with plasmids encoding ABCB6-FLAG, ABCB6-V5, or indicated K629G mutants. The immunoprecipitation was performed as described in the Fig. 2.1. 
this motif in ABCB6 dimerization. NIH3T3 cells were transiently co-transfected with ABCB6-Flag and mutant constructs as indicated. Anti-Flag antibody was used to immunoprecipitate $\mathrm{ABCB} 6-$ Flag from lysates and the interaction between mutant ABCB6 proteins and wild type ABCB6 was analyzed by immunoblotting using anti-V5 antibody (Fig. 2.3.). As shown in Fig. 2.3, all the mutants co-immunoprecipitated with wild type ABCB6 indicating that neither of the motifs are critical for ABCB6 dimerization. Moreover, the substrate binding was retained in G508/512L mutant as shown by the hemin-agarose pull-down assay (Fig. 2.4.). Therefore, GXXXG motif is unlikely to affect the packing of TM helices, unlike BCRP where transport activity was abolished [95].

\subsubsection{Interaction between $\mathrm{ABCB6}$ and Heme Is Disrupted by Tetrapyrroles}

$\mathrm{ABC}$ transporters translocate structurally different classes of compounds; however, the substrate specificity cannot be predicted from the amino acid sequence at present due to little structural information and thus, needs to be tested directly by determining binding affinity or by measuring transport activity. Other authors of this study showed that ABCB6 binds to hemin conjugated to agarose beads. The binding was specific because a naturally occurring splice variant of $\mathrm{ABCB} 6$, which lacks 50 amino acids but still localizes to mitochondria, did not bind to hemin-agarose. Therefore, I next tested whether other compounds with a tetrapyrrole ring would displace ABCB6 from hemin-agarose (Fig. 2.5.). Cell lysates were prepared from NIH3T3 cells transiently expressing ABCB6-V5 and were incubated with indicated compounds before heminagarose pull-down assays. Notably, CPIII, an oxidized form of heme precursor CPgenIII that needs to be shuttled into the mitochondria from the cytoplasm, was most potent in displacing ABCB6 from hemin-agarose. Commercially available CPIII was used instead of physiologically relevant CPgenIII because CPgenIII is readily oxidized to CPIII in normal conditions. Another heme precursor, PPIX, also competed for binding to ABCB6 at a higher concentration. On the other hand, PBG, a monopyrrole heme precursor, had no effect on ABCB6 binding to hemin-agarose even at the highest concentration tested $(80 \mu \mathrm{M})$. PhA, a chlorophyll metabolite with a tetrapyrrole backbone, was effective in displacing $\mathrm{ABCB} 6$ from hemin-agarose at $10 \mu \mathrm{M}$, whereas vitamin $\mathrm{B}_{12}$, a tetrapyrrole ring with a bulky side chain, was not, even at the highest concentration. These results show that ABCB6 preferentially binds compounds with a tetrapyrrole structure with anionic propionate side chains.

\subsubsection{ABCB6 Transports Porphyrins in an ATP-Dependent Manner}

Transport cycles by ABC transporters are fueled by ATP hydrolysis. The transport process by $\mathrm{ABC}$ transporters begins with the binding of their substrates at MSDs and ends with the stimulation of ATPase activity at NBDs resetting the transporter for the next cycle. Because various porphyrins bind to ABCB6, we next tested whether they can stimulate ATPase activity of ABCB6 and if they are transported into isolated mitochondria in an ATP-dependent manner. Since a mutation of the conserved lysine 

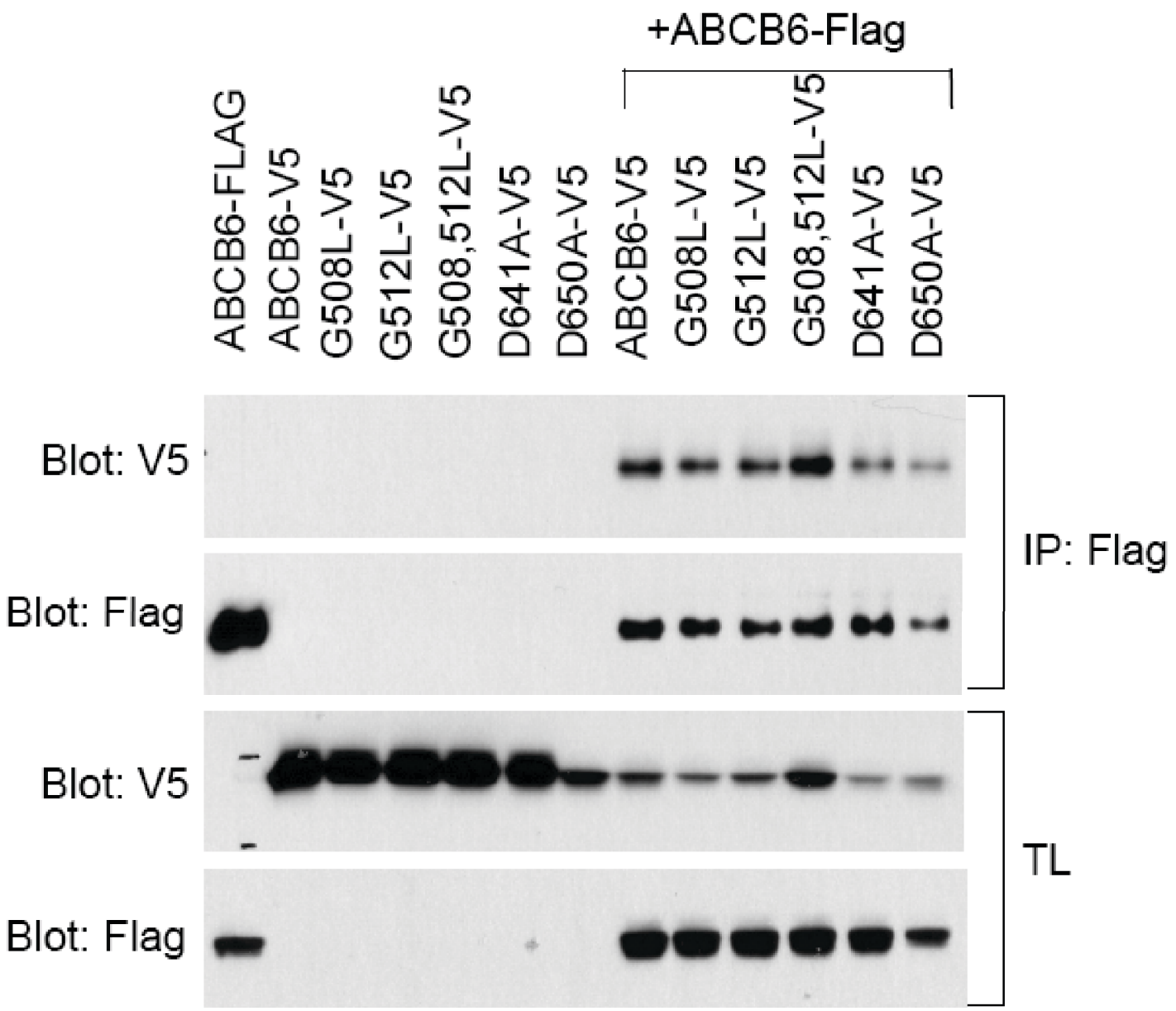

Figure 2.3. GXXXG and the $D(X)_{8} D$ domains are not critical for the dimerization. NIH 3 T3 cells were transiently transfected with plasmids encoding ABCB6-FLAG, ABCB6-V5, or indicated mutants, and immunoprecipitation was performed as described in Fig. 2.1. All the mutants interacted with ABCB6. TL, total lysate. 


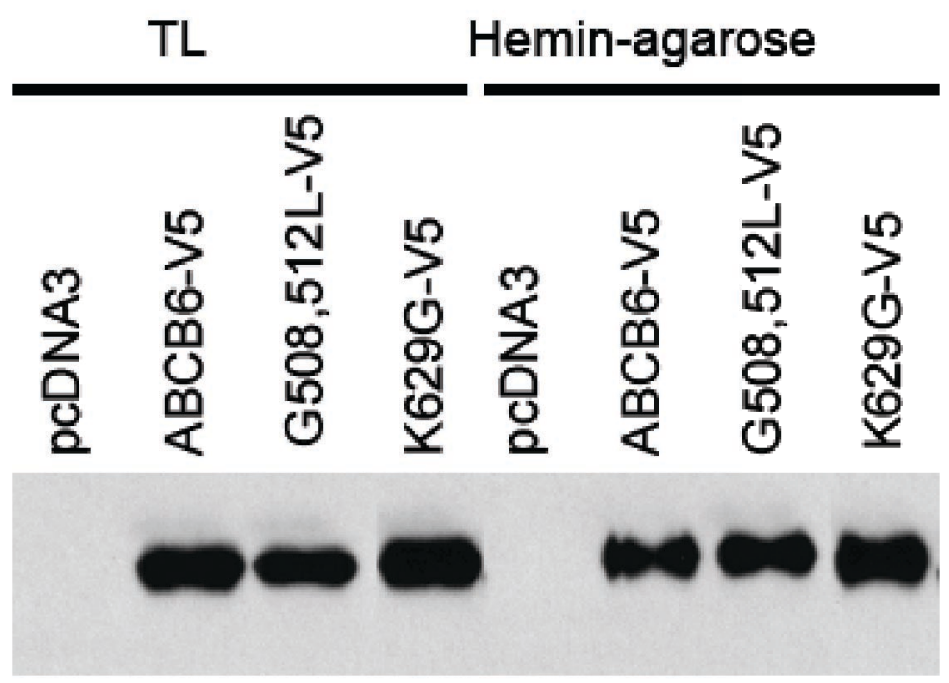

Figure 2.4. Walker A mutant and GXXXG mutant bind hemin-agarose.

Hemin-agarose pull-down assay was performed using lysates prepared from NIH3T3 cells transiently transfected with indicated plasmids. TL, total lysate. 


\section{$0 \quad 1040 \quad 80 \quad(\mu \mathrm{M})$

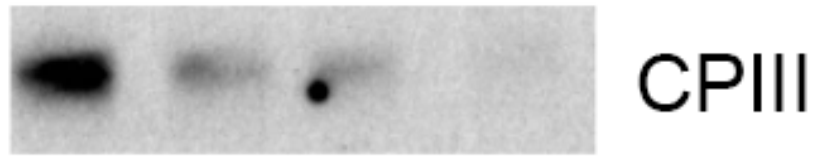

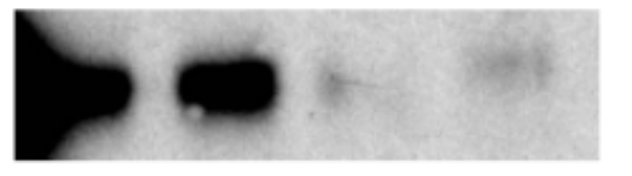

Hemin

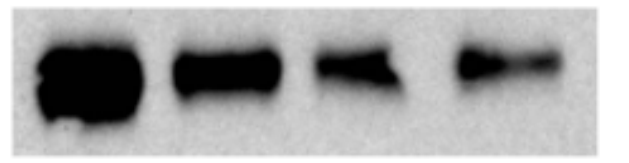

\section{PP IX}

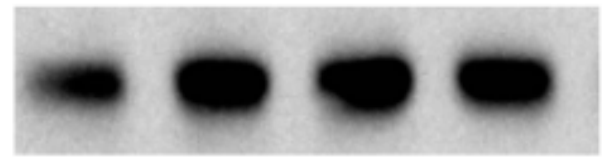

PBG

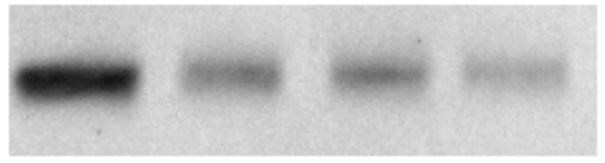

\section{PhA}

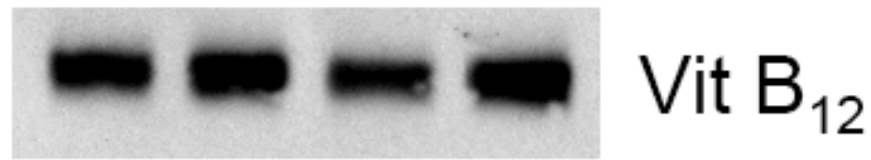

Figure 2.5. CPIII, PPIX, and PhA displace ABCB6 from hemin-agarose.

Cell lysates from NIH3T3 cells transiently transfected with ABCB6-V5 were preincubated with indicated amounts of compounds prior to hemin-agarose pull-down assays. ABCB6 bound to hemin-agarose was analyzed by immunoblotting using anti-V5 antibody. The incubation with CPIII, hemin, PP IX, and PhA (tetrapyrroles) decreased the amount of $\mathrm{ABCB} 6$ in hemin-agarose pull-down fraction, whereas, vitamin $\mathrm{B}_{12}$ and a monopyrrole, porphobilinogen had no effect. CPIII; coproporphyrin III, PP IX; protoporphyrin IX, PBG; porphobilinogen, PhA; pheophorbide a, Vit $\mathrm{B}_{12}$; vitamin $\mathrm{B}_{12}$. 
residue in the Walker A motif impairs the transport activity of many $\mathrm{ABC}$ transporters, we decided to use the Walker A mutant, ABCB6-K629G-V5 as a control in these experiments. I generated K562 cell lines that stably expressed either ABCB6-V5 or ABCB6-K629G-V5 using G418 selection and selected for clones that expressed comparable amounts of proteins. Crude mitochondrial preparations from these cell lines were tested for $\mathrm{ABCB} 6$ and the mutant protein and the results indicate the mutant protein, as well as the wild-type ABCB6, is enriched in this mitochondrial fraction (Fig. 2.6.). Vanadate-sensitive ATPase activity was measured in these mitochondrial preparations as a release of inorganic phosphates using a colorimetric assay. CPIII-stimulated ATPase activity was observed with mitochondria expressing ABCB6 but not with those expressing the Walker A mutant protein (Fig. 2.6.). Further, other authors of this study showed that the uptake of ${ }^{55} \mathrm{Fe}-$ labeled hemin by mitochondria expressing ABCB6 was ATP- and temperature-dependent suggesting that the transport requires ATP hydrolysis. The transport activity was abolished in the mitochondria expressing the Walker A mutant, which retained the heme-binding ability (Fig. 2.4.), further supporting that ABCB6 transports porphyrins into mitochoncria in an ATP dependent manner. A tetrapyrrole, CPIII, but not a monopyrrole, PBG, competed for hemin uptake into mitochondria. Taken together, CPIII showed a strong competition for binding of heminagarose to ABCB6, stimulated ATPase activity, and inhibited hemin uptake into mitochondria.

\subsubsection{ABCB6 Increases the Rate of Porphyrin Biosynthesis}

ABCB6 is induced by both erythroid differentiation and increased intracellular porphyrin levels. Because ABCB6 levels appear to be coordinated with heme biosynthesis, we next tested the role of ABCB6 in heme biosynthesis. Using K562 stable cell lines, I was able to show an elevated basal PPIX levels in cells expressing the wildtype ABCB6 compared to K562 parental cells or cells expressing the Walker A mutant (Fig. 2.7.). To determine whether the higher PPIX accumulation was due to an increased rate of de novo heme biosynthesis in ABCB6 expressing cells, other authors of this study determined the amount of radiolabeled glycine incorporated into porphyrins. In both mouse and human erythroid cell lines, Mel and K562, respectively, cells expressing ABCB6 exhibited higher rate of de novo porphyrin biosynthesis. In CHO-TRex system where gene expression can be induced by the addition of tetracycline, ABCB6 expressing cells had a higher rate of de novo heme biosynthesis compared to the Walker A mutant expressing cells. A loss of one Abcb6 allele in 129/SVJ-derived embryonic stem (ES) cell lines resulted in a lower PPIX levels compared to $\mathrm{Abcb}^{+/+}$cells when treated with ALA, suggesting a lower porphyrin biosynthesis in cells lacking one Abcb6 allele.

\subsection{Discussion}

Previously, mitochondrial half transporter ABCB6 was implicated in cellular iron homeostasis and was suggested to be the functional homolog of yeast Atm1p [171]. However, phylogenetic analyses and the functional studies showed that $\mathrm{ABCB} 7$ is the 


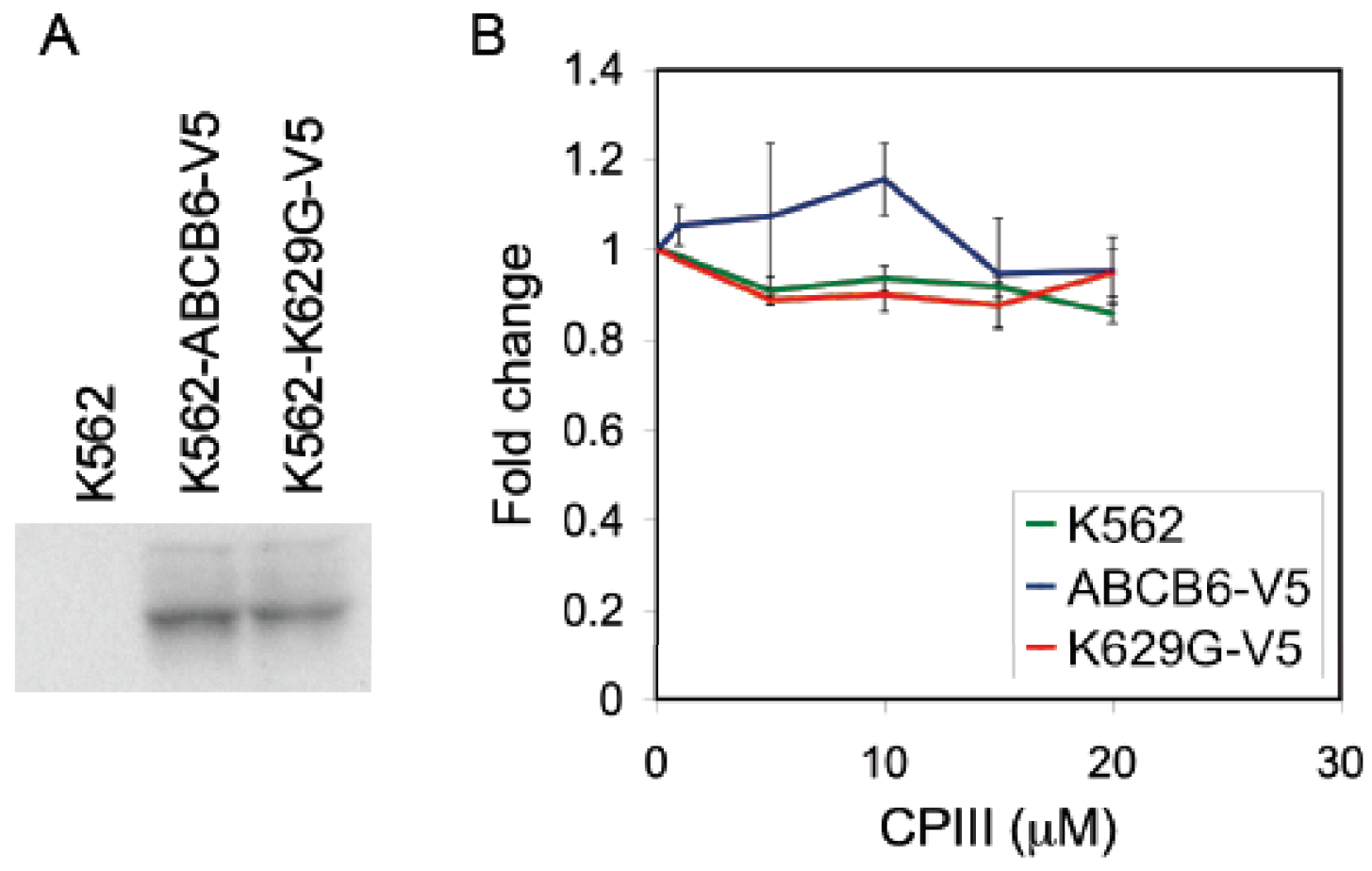

Figure 2.6. CPIII stimulates ATPase activity in ABCB6 expressing mitochondria. A) Both wild type and the Walker A mutant are enriched in the crude mitochondria fraction. Crude mitochondria preparations were isolated from K562 cells stably expressing either ABCB6-V5 or the Walker A mutant using differential centrifugation and analyzed by immunoblotting using anti-V5 antibody. B) Vanadate sensitive ATPase activity was measured as inorganic phosphate release. Crude mitochondria preparations from K562 cells stably expressing ABCB6 or the Walker mutant was incubated with varying amounts of CPIII. The values are expressed as measurements \pm S.E.M. 


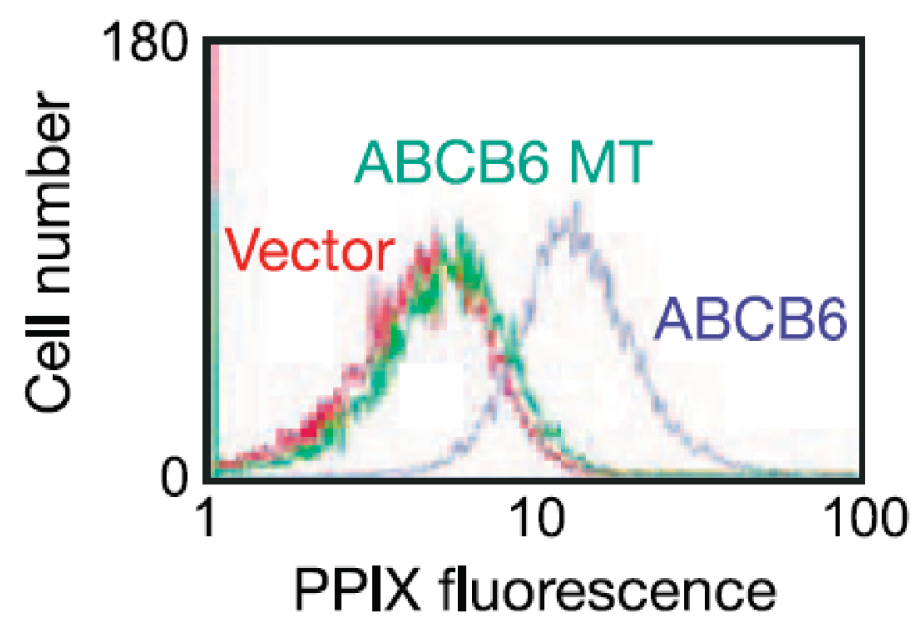

Figure 2.7. ABCB6 expressing cells exhibit higher intracellular PPIX levels.

The intracellular PPIX levels in the K562 cells expressing vector, ABCB6 or the Walker A mutant (ABCB6 MT) were measured by FACS analysis. 
functional orthlog of Atm1p [159]. Therefore, the physiological role and the substrates of ABCB6 remained elusive. The high expression levels of ABCB6 in fetal liver and erythroid-lineage cells, its mitochondrial localization, and coordinated gene expression with heme biosynthesis and erythroid differentiation led us to identify the role of ABCB6in heme biosynthesis. In this study, I showed that ABCB6 is a homodimer that binds to various porphyrins and that its ATPase activity is stimulated by a porphyrin, CPIII. Furthermore, generation of a cell line stably expressing the Walker A mutant ABCB6 enabled us to distinguish porphyrin binding from the transport activity involving ATP binding and hydrolysis. The elevated basal levels of PPIX were seen in cells expressing wild type ABCB6 but not in the cells expressing the Walker A mutant showing that the transport function of $\mathrm{ABCB} 6$ is required for the observed effect. Our results suggest that $\mathrm{ABCB} 6$ is ideally located on the mitochondrial outer membrane to transport a heme precursor, CPgenIII, into the intermembrane space to overcome a possible rate-limiting step, thereby facilitating heme biosynthesis.

The affinity for porphyrins, mitochondrial uptake of hemin, and CPIII-stimulated ATPase activity in ABCB6-expressing cells all demonstrate that porphyrins are ABCB6 substrates. The affinity of ABCB6 for heme-related porphryins was highest for CPIII, followed by hemin, and then by PPIX. Especially, an oxidized form of a heme intermediate CPgenIII, CPIII, displaced more than 90\% of ABCB6 from hemin-agarose at $10 \mu \mathrm{M}$. The monopyrrole PBG did not disrupt the interaction between ABCB6 and hemin-agarose. Notably, a plant tetrapyrrole and a mitochondrial toxin, pheophorbide a also displaced ABCB6 from hemin-agarose effectively. Generally, the ATPase activity of $\mathrm{ABC}$ transporters is induced by a substrate binding. Therefore, the observation that ATPase activity is induced in the CPIII-treated ABCB6 expressing mitochondria further supports CPIII, and possibly other porphyrins as ABCB6 substrates. Further, the uptake of hemin into ABCB6 expressing mitochondria conclusively shows that the porphyrins are ABCB6 substrates. The temperature and ATP dependence of the hemin uptake suggests that ATP hydrolysis fuels the transport cycle. Accordingly, the Walker A mutant, which lacks ATPase activity, failed to transport hemin. Moreover, by using the Walker A mutant, which still interacted with hemin-agarose, we excluded the possibility that the increased ${ }^{55} \mathrm{Fe}$-hemin in the ABCB6 expressing mitochondria was simply due to the binding of hemin to ABCB6 at the mitochondrial membrane. These results indicate that heme and other porphyrins are substrates of ABCB6.

The basal PPIX levels are increased in ABCB6-expressing cells compared to the vector cells, and accordingly the accelerated rate of de novo porphyrin biosynthesis was confirmed. In contrast, cells expressing the Walker A mutant ABCB6 behaved much like vector cells and no increase in the PPIX levels nor the de novo porphyrin synthesis was observed. These results indicate that the effect of ABCB6 on heme biosynthesis is dependent on its transport function. In this study, we showed that ABCB6 interacts with porphyrins and localizes on the mitochondrial outer membrane. Furthermore, the Cterminal tail of $\mathrm{ABCB} 6$ resides in the cytoplasm, which strongly suggests that $\mathrm{ABCB} 6$ transports its substrates from the cytoplasm into the intermembrane space. Hemin uptake by ABCB6-expressing mitochondria also indicates the same direction of transport. From these results, we postulated that ABCB6 is an ideal candidate for shuttling the heme 
precursor, CPgenIII, into the mitochondria, thus facilitating the heme biosyntheis. The proposed role of $\mathrm{ABCB} 6$ as a CPgenIII transporter explains the accelerated rate of heme biosynthesis because moving the CPgenIII into the intermembrane space, where the next enzyme in the pathway resides, may overcome the possible rate-limiting step. Moreover, our finding that PPIX levels are lower in the Abcb6 ${ }^{+/-}$ES cells after ALA treatment is consistent with the role of ABCB6 in heme biosynthesis. Taken together, these results strongly suggest CPgenIII as one of the physiological substrates for ABCB6.

ABCB6 homodimerizes, and therefore, is unlikely to require a heterodimeric partner. This finding was important because some $\mathrm{ABC}$ half transporters form obligate heterodimers and the co-expression of heterodimeric partner is required for the proper localization and the function [57]. The overexpression of a single protein ABCB6 was sufficient to increase the PPIX levels and enhance mitochondrial hemin uptake, which is consistent with ABCB6 functioning as a homodimer. Several domains are reported to be important for the dimerization of $\mathrm{ABC}$ half transporters or other membrane proteins. The yeast $\mathrm{ABC}$ transporter Atm1p failed to form a stable homodimer when the conserved lysine residue in the Walker A motif was mutated [94]. Another motif implicated in the packing of $\alpha$-helices, GXXXG, is studied in several membrane proteins including ABC transporters $[95,215,216]$. Mutation of the GXXXG in BCRP did not impair dimer formation, although its transport function was altered [95]. A domain that is similar to a downstream region of the Walker A motif of several ABC transporters is a critical domain for the homodimerization of the yeast protein Gts1p [221]. Moreover, the mutation of the aspartate residues in the domain (DXXXGXXXXD) resulted in a loss or a diminished interaction between $\mathrm{C}$-terminal regions of yeast $\mathrm{ABC}$ transporter Mdllp and Gts1p [221]. Generation of mutant ABCB6 that is impaired in the dimer formation would be useful to test whether the dimerization is required for substrate binding, localization, and the function. All of the mutants tested (Walker A, GXXXG, and DXXXGXXXXD) interacted with the wild type $\mathrm{ABCB} 6$ protein indicating that none of these domains are critical for the homodimerization of ABCB6. However, it is possible that the multiple domains are involved in the formation of a stable dimer and that a loss of one domain does not immediately result in a lack of dimer formation. Furthermore, because the conserved lysine residue in the Walker A motif and the DXXXGXXXXD motif are located in the NBD, mutations in these domains may affect the formation of NBD dimer rather than the overall dimerization, which may result in an altered ATP binding and hydrolysis.

In this study, we demonstrate that the mitochondrial $\mathrm{ABC}$ transporter $\mathrm{ABCB} 6$ is a porphyrin transporter on the mitochondrial outer membrane. Because ABCB6 positively regulates heme biosynthesis, we speculate that $\mathrm{ABCB} 6$ transports $\mathrm{CPgenIII}$ into the mitochondrial intermembrane space where it is then converted into PPgenIX. The transmembrane movement of the anionic porphyrin CPgenIII has been suggested to involve an ATP-dependent active transport. Therefore, ABCB6, ideally located on the mitochondrial outer membrane, may be the elusive porphyrin transporter required to overcome the possible rate-limiting step in heme biosynthesis. The coordinated upregulation of $\mathrm{ABCB} 6$ during erythroid differentiation and elevated porphyrin biosynthesis is consistent with its role in heme biosynthesis. ABCB6 may transport heme 
into the mitochondria in order to supply heme for mitochondrial hemoproteins, whereas compartmentalization of porphyrins may protect plasma membrane and other cellular components from their cytotoxic effects. Like other ABC transporters, we anticipate ABCB6 to transport a wide array of substrates; however, the current finding on its porphyrin transport and its role in heme biosynthesis delineates its unique physiological role. 


\section{CHAPTER 3. ABCB6 PROVIDES INSIGHTS INTO THE MECHANISM FOR SUBSTRATE TRANSPORT BY ABC TRANSPORTERS}

\subsection{Introduction}

$\mathrm{ABC}$ transporters constitute a large protein superfamily, which is involved in the transport of a wide array of substrates including xenobiotics and biological metabolites across lipid bilayers [2]. The transport of various drugs is the basis for the multidrug resistance phenotype conferred by an overexpression of certain $\mathrm{ABC}$ transporters, whereas many others are implicated in the homeostasis of biomolecules, thus being identified as disease causing genes in many human disorders. For transport activity, ABC transporters require two MSDs and two NBDs. Half transporters, which consist of one NBD and one MSD, therefore, need to either homo- or hetero-dimerize in order to reconstitute transport activity. MSDs, generally composed of six TM helices, function in substrate binding and determine the substrate specificity. The transport of substrates by ABC transporters is energy-dependent and is linked to ATP binding and/or ATP hydrolysis at NBDs.

In order to understand how the transport cycle is driven by NBDs, extensive biochemical and structural studies have been performed using mutants and nucleotide analogs to trap $\mathrm{ABC}$ transporters in different states during the transport cycle. Crystal structures of isolated NBDs demonstrated that ATP-bound NBDs form a stable dimer, where two ATP molecules are sandwiched by the two NBDs, providing the basis for the requirement of two NBDs [110]. The NBDs are highly conserved among ABC transporters and contain motifs such as Walker $\mathrm{A}$ and $\mathrm{B}, \mathrm{ABC}$ signature, $\mathrm{H}$ and $\mathrm{Q}$ loops [111]. The structures of isolated NBDs showed that ATP is bound by Walker A and B motifs from one NBD and by ABC signature motif from the other [110]. Substitution of the conserved lysine residue in the Walker A motif impaired ATP binding in some and ATP hydrolysis in other ABC transporters [111, 212, 213, 222, 223]. On the other hand, substitution of the conserved glutamate residue in the Walker B motif abolished ATPase activity and subsequent release of ADP and Pi to confirm its role in ATP hydrolysis, thus making this mutation useful when studying the structure of nucleotide-bound NBD [213]. The position of ATP between two NBDs explains the requirement for having two functional NBDs to complete the transport cycle including ATP hydrolysis. Previous studies suggest that the two NBDs are required for ATP binding and hydrolysis but are not equivalent in some transporters, i.e., one NBD has a higher affinity for ATP and other has a higher ATPase activity. This seems to be the case for some full transporters such as ABCC1 [222], as well as half transporters that form heterodimers including TAP1/ABCB2 and TAP2ABCB3 [111]. However, for Md11, a homodimeric mitochondrial half transporter, such asymmetry does not appear to exist and the ATP binding and hydrolysis occur in a stepwise manner [224].

A number of studies have established a coordinated action between MSDs and NBDs in the substrate translocation by ABC transporters. Although there may be some variations among different $\mathrm{ABC}$ transporters, the transport cycle is initiated upon binding 
of a substrate(s) to the MSDs, followed by ATP binding or ADP to ATP exchange at the NBDs. It has been proposed that the subsequent ATP hydrolysis provides the energy to translocate the substrate to the other side of the lipid bilayers [113, 115]. However, recent biochemical and structural studies have implicated another mechanism for ATP-driven substrate transport [97, 114]. Sometimes referred to as "ATP switch model," the model postulates that MSDs alternate between high-affinity state and low-affinity state for a substrate and that the ATP binding and ATP hydrolysis trigger the conformational changes to allow for releasing the substrate and resetting the transporter, respectively [114]. A substrate binds to the MSDs at a high-affinity substrate site. ATP binding to the NBDs induces a formation of a closed NBD dimer, which is coupled to conformational changes in the MSDs resulting in a switch to a low-affinity substrate binding state, thus translocating the substrate. Subsequent ATP hydrolysis disengages the NBD dimer, thereby resetting the $\mathrm{ABC}$ transporter to a high-affinity state to start a new transport cycle. Indeed, studies show that ATP binding to transporters such as Pgp induces a conformational change as shown by different patterns of tryptic digestion [225]. In addition, radiolabeled ligand binding assays have shown that MRP1 adapts a low-affinity substrate site in the presence of nucleotides [226]. However, much of the structural studies regarding nucleotide-bound NBDs have used isolated NBDs so how the changes in NBDs transmit the information to MSDs is speculative [110,111]. Variations appear to exist within the two proposed transport models among the transporters. Nevertheless, understanding transport mechanism is important to develop approaches to block transporter functions. Since some half transporters confer resistance to drug therapy, namely $\mathrm{ABCG} 2 / \mathrm{BCRP}$, it is important to understand the transport mechanism in half transporters that form homodimers, which would have homologous NBDs. ABCB6 is another half-transporter, shown to be a homodimer, and is localized to the mitochondrial outer membrane. ABCB6 transports heme precursors/porphyrins into mitochondria, thereby facilitating heme biosynthesis [204]. Since ABCB6 binds to heme conjugated to agarose beads, it is an ideal model to study substrate binding and also the transport mechanism.

In this study, we used ABCB6 and its Walker A lysine mutant to determine the effect of nucleotide binding on substrate affinity. The conserved lysine residue in the Walker A motif is critical for ATP binding and /or hydrolysis of ABC transporters and is also shown to be important for the homodimerization of yeast mitochondrial ABC transporter, Atm1p. We have shown that the Walker A mutant lacks ATP-dependent heme transport activity and that the lysine mutation does not affect the homodimerization of ABCB6. In this study, we show that the substrate binding, assessed by using hemin conjugated to agarose beads, is affected in the Walker A mutant only modestly. However, the affinity for ATP was drastically reduced. We determined the effect of ATP and AMPPNP binding to ABCB6-hemin-agarose complex and provided insights into the role of nucleotide binding in conformational changes in MSDs. 


\subsection{Experimental Procedures}

\subsubsection{Cell Culture and Transfection}

NIH3T3 cells were cultured in Dulbecco's modified Eagle's medium (DMEM) containing $4500 \mathrm{mg} / \mathrm{L}$ glucose supplemented with 10\% FCS, $2 \mathrm{mM}$ L-glutamine, and

antibiotics (Gibco). Cells were plated on 6 well-clustered dish at $3 \times 10^{5}$ cells/well 24 hours prior to transfection and were transfected using LipofectAMINE Plus (Invitrogen) according to the manufacturer's protocols.

\subsubsection{Site-Directed Mutagenesis}

pcDNA3.1-hABCB6-V5-His plasmid was designed to express human ABCB6 with a C-terminal Flag tag. A point mutation was introduced to substitute the lysine residue at 629 in Walker A motif with a glycine residue using a Quikchange site-directed mutagenesis kit (Stratagene) and primers (K629G sense; 5'- GTGGGCCCATCTGGGGCAGGGGGGAGCACAATTTTGCGCCTGCTG-3', K629G antisense; 5' - CAGCAGGCGCAAAATTGTGCTCCCCCCTGCCCCAGATGGGCCCAC-3'). All genes were thoroughly sequenced after mutagenesis.

\subsubsection{Immunoblotting}

NIH3T3 cells were transiently transfected with expression plasmids indicated in the legends. Twenty-four hours after transfection, cells were washed with phosphate buffered-saline (PBS), scraped into ice-cold PBS containing protease inhibitor cocktail (complete EDTA free, Roche), and resuspended in buffer A (50 mM Tris- $\mathrm{HCl}$ (pH 7.5), $150 \mathrm{mM} \mathrm{NaCl}, 10 \%$ glycerol, and $1 \mathrm{x}$ protease inhibitor cocktail). The samples were then sonicated and the final concentration of $1 \%$ Nonidet P40 added and solubilized by constant rocking for $1 \mathrm{hr}$. The samples were centrifuged at $16,000 \mathrm{x}$ g for $15 \mathrm{~min}$ at $4{ }^{\circ} \mathrm{C}$ to remove cell debris. The protein concentration was quantified by Bradford method (Protein assay, Bio-Rad) and lysate was further subjected to immunoblotting or pulldown assays. For immunoblotting, samples were subjected to 10\% Laemmli SDS-PAGE and transferred to a nitrocellulose membrane (Amersham Pharmacia). The blot was blocked for $2 \mathrm{hr}$ with blocking buffer (10\% skim milk in PBS containing $0.1 \%$ Tween 20 , PBS-T), incubated with anti-V5 polyclonal (MBL, Japan) antibody for $2 \mathrm{hr}$, and washed four times with PBS-T. Subsequently, the blot was incubated with anti-rabbit or mouse IgG conjugated to horse radish peroxidase (Amersham Pharmacia) for $1 \mathrm{hr}$, followed by washing with PBS-T for four times. The bands were detected using enhanced chemiluminescence detection kits (ECL, Amersham Pharmacia). 


\subsubsection{Hemin-Agarose or ATP-Agarose Pull-Down Assays}

Cell lysates prepared from cells transfected with ABCB6-V5 or ABCB6-K629GV5 expression plasmids were incubated with hemin conjugated to agarose beads (Sigma) or ATP-agarose (N6, Sigma) for $30 \mathrm{~min}$ or indicated times at $24^{\circ} \mathrm{C}$. The samples were washed with wash buffer and analyzed by immunoblotting using anti-V5 polyclonal antibody. The intensity of the bands was analyzed by densitometry using Image $\mathbf{J}$ (http://rsb.info.nih.gov/ii).

\subsubsection{Nucleotide-Induced ABCB6 Dissociation}

Cell lysates were incubated with hemin-agarose for $30 \mathrm{~min}$ at $24^{\circ} \mathrm{C}$ to form ABCB6-hemin-agarose complex. The resin was washed once and resuspended in $200 \mu \mathrm{l}$ buffer A. The indicated amounts of ATP or AMPPNP were added to the samples. After 30 min incubation at $24^{\circ} \mathrm{C}$, the amounts of $\mathrm{ABCB} 6$ protein remained bound to the resin were analyzed by immunoblotting and densitometry.

\subsection{Results}

\subsection{1. $A B C B 6$ and $A B C B 6^{K 629 G}$ Display Similar Kinetics for Hemin Binding}

Transport activity/mechanism of ABC transporters has been studied rigorously using mutant forms of proteins in which critical residues involved in the ATP binding and/or ATP hydrolysis have been substituted with other amino acids, which result in a loss of function [213, 222]. We have previously generated an ABCB6 mutant construct in which the conserved lysine residue in the Walker A motif is substituted with a glycine residue $\left(\mathrm{ABCB} 6^{\mathrm{K} 629 \mathrm{G}}\right.$ ) (Fig. 2.2.). In accordance with previous reports on the other $\mathrm{ABC}$ transporters, $\mathrm{ABCB} 66^{\mathrm{K} 629 \mathrm{G}}$ mutant protein lacks heme transport activity and cells expressing $\mathrm{ABCB} 6^{\mathrm{K} 629 \mathrm{G}}$ do not accumulate PPIX, an indicator of functional $\mathrm{ABCB} 6$ [204]. Moreover, the mutant ABCB6 homodimerizes. To determine the substrate binding to $\mathrm{ABCB} 6^{\mathrm{K} 629 \mathrm{G}}$ mutant, hemin-agarose pull-down assays were perfomed. Lysates from $\mathrm{NIH} 3 \mathrm{~T} 3$ cells transiently transfected with $\mathrm{ABCB} 6-\mathrm{V} 5$ or $\mathrm{ABCB} 6^{\mathrm{K} 629 \mathrm{G}}-\mathrm{V} 5$ were incubated with hemin conjugated to agarose beads, and proteins bound to the heminagarose were analyzed by immunoblotting (Fig. 2.5.). ABCB6 ${ }^{\mathrm{K} 629 \mathrm{G}}-\mathrm{V} 5$ mutant effectively interacted with hemin-agarose at equilibrium, suggesting that the conformation of substrate (hemin) binding site is not altered grossly by a mutation in the NBD. Binding of ABCB6 to hemin-agarose is specific as pre-incubation of lysates with hemin $(80 \mu \mathrm{M})$ can compete for the binding and a deletion mutant of ABCB6 does not bind to hemin-agarose [204].

To further characterize the binding of hemin-agarose to $\mathrm{ABCB} 6$ and $\mathrm{ABCB} 6^{\mathrm{K} 629 \mathrm{G}}$ at equilibrium, different amounts of hemin-agarose were incubated with lysates for the pull-down assays, and intensity of the bands from immunoblots was quantified by 
densitometry and plotted as a function of hemin-agarose concentration. The binding of $\mathrm{ABCB} 6$ or $\mathrm{ABCB} 6^{\mathrm{K} 629 \mathrm{G}}$ to hemin-agarose was dependent on the amount of heminagarose added as shown in Fig. 3.1.A.

Binding of hemin to $\mathrm{ABCB} 6^{\mathrm{K} 629 \mathrm{G}}-\mathrm{V} 5$ was further characterized by determining the association kinetics. Hemin-agarose pull-down assays were performed at different time points, and intensity of the bands from Western blot was quantified by densitometry and plotted as a function of time (Fig. 3.1.B). Non-linear regression equation was used to perform the curve fitting. There was no significant difference between ABCB6-V5 and $\mathrm{ABCB} 6^{\mathrm{K} 629 \mathrm{G}}-\mathrm{V} 5$ on forming a complex with hemin-agarose.

\subsubsection{Loss of the Walker A Lysine Results in Reduced ATP Binding}

The substitution of conserved lysine residue in the Walker A motif of ABCB6 did not affect the dimerization and modestly reduced the rate of ABCB6 association with hemin. Therefore, the loss of function observed with $\mathrm{ABCB} 6^{\mathrm{K} 629 \mathrm{G}}$ is due either to changes in the levels of ATP binding or ATP hydrolysis, both of which are critical to transport activity. To determine the ATP binding of ABCB6 and $\mathrm{ABCB} 6^{\mathrm{K} 629 \mathrm{G}}$, an ATPagarose pull-down assay was performed at $24^{\circ} \mathrm{C}$. ABCB6-V5 bound to ATP-garose in a concentration dependent manner, whereas the majority of $\mathrm{ABCB} 6^{\mathrm{K} 629 \mathrm{G}}-\mathrm{V} 5$ was recovered in the flow through even at the highest concentration of ATP-agarose used (Fig. 3.2.A).

In order to identify whether the loss of affinity for ATP observed with the mutant protein was unique to lysine to glycine substitution, the homology modeling of NBD from ABCB6 with several substitutions was performed by Jufang Shan using Hlyb, $\alpha-$ hemolysin translocation ATP-binding protein, bound to ATP as a template (Fig. 3.2.B, only the NBDs with $\mathrm{K}, \mathrm{K}$ to $\mathrm{G}$, and $\mathrm{K}$ to $\mathrm{M}$ are shown). In both the mutants, the favorable interaction between the phosphate group from ATP and the side chain of the lysine is lost. When free energy for ATP binding was calculated for each mutants $(\mathrm{G}, \mathrm{M}, \mathrm{A}, \mathrm{S}, \mathrm{T}$, and $\mathrm{Q}$ ), all the mutants had a positive $\Delta \Delta \mathrm{G}$ value compared to the wild type (K) suggesting that the ATP binding is unfavorable for these mutants. However, when $\mathrm{ABCB} 6^{\mathrm{K} 629 \mathrm{G}}$-V5 is incubated with ATP-agarose at $37^{\circ} \mathrm{C}$, small amount of ABCB6 ${ }^{\mathrm{K} 629 \mathrm{G}}$ V5 binds to the beads (Fig. 3.2.C). Therefore, the loss of function seen with $\mathrm{ABCB} 6^{\mathrm{K} 629 \mathrm{G}}-\mathrm{V} 5$ can be attributed to its significantly reduced affinity for ATP. In accordance with the calculated $\Delta \Delta \mathrm{G}$ values for ATP binding of the other mutant NBDs, substitution of the conserved lysine residue to $\mathrm{M}, \mathrm{A}, \mathrm{S}, \mathrm{T}$, and $\mathrm{Q}$ also resulted in a reduced affinity for ATP (Fig. 3.2.D).

\subsubsection{ATP Binding to ABCB6 Changes Its Affinity for Hemin-Agarose}

According to the ATP-swith model, release of a substrate from ABC transporters requires a conformational change from a high affinity substrate binding state to a low affinity state [114]. If ATP binding is sufficient to induce this conformational change in 
A

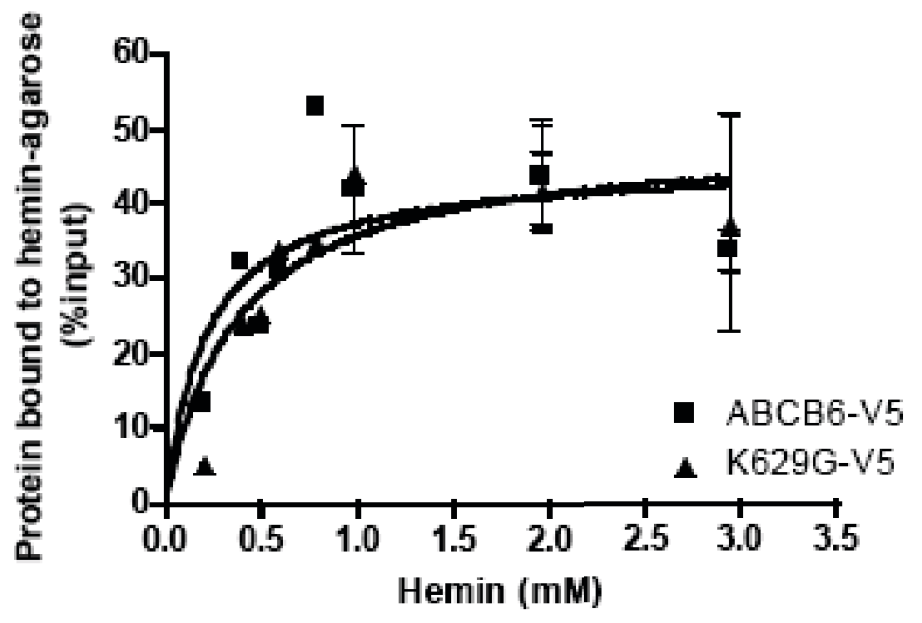

$\mathrm{B}$

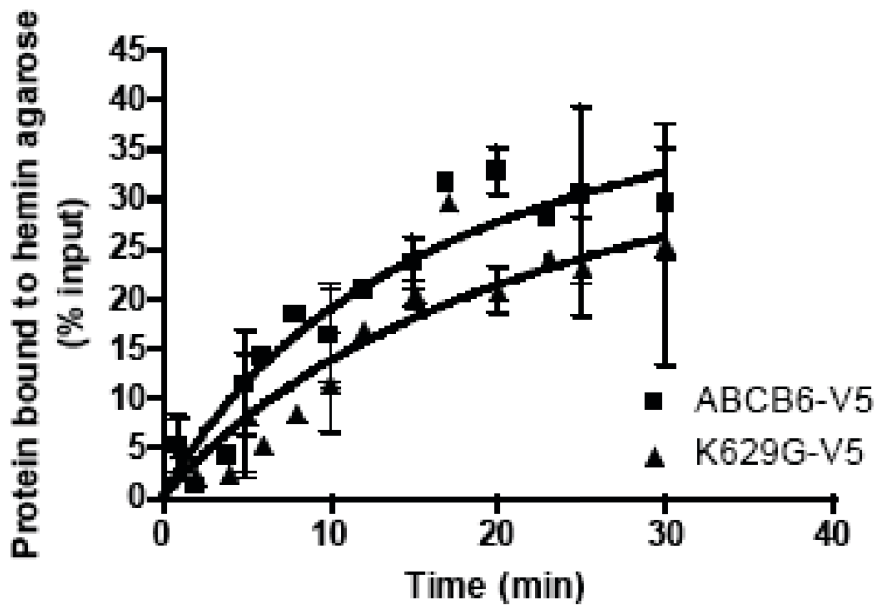

Figure 3.1. $\mathrm{ABCB} 6$ and $\mathrm{ABCB} 6^{\mathrm{K} 629 \mathrm{G}}$ binds to hemin-agarose with similar kinetics. A) $\mathrm{ABCB} 6$ and $\mathrm{ABCB} 6^{\mathrm{K} 629 \mathrm{G}}$ show similar $\mathrm{B}_{\max }$ and $\mathrm{k}_{\mathrm{d} \text { obs. }}$. Cell lysates prepared from NIH 3 T3 cells transiently transfected with a plasmid encoding ABCB6-V5 or $\mathrm{ABCB} 66^{\mathrm{K} 629 \mathrm{G}}$ were incubated with varying amounts of hemin-agarose beads at $24^{\circ} \mathrm{C}$. Hemin-agarose beads were washed twice, and the samples were analyzed by immunoblotting. B) The association kinetics is similar for the wild type and mutant ABCB6. Cell lysates were incubated with hemin-agarose for indicated times at $24^{\circ} \mathrm{C}$. The values are measurement \pm S.D. 
Figure 3.2. ATP-binding is severely impaired in Walker A mutant ABCB6.

A) Cell lysates were prepared from NIH 3T3 cells transiently transfected with a plasmid encoding $\mathrm{ABCB} 6-\mathrm{V} 5$ or $\mathrm{ABCB} 6^{\mathrm{K} 629 \mathrm{G}}$ and incubated with varying amounts of ATPagarose beads (conjugated at N-6) at $24^{\circ} \mathrm{C}$. ATP-agarose beads were washed twice, and the samples were analyzed by immunoblotting. Flow through from the pull-down assays was TCA precipitated and also analyzed. B) NBDs from ABCB6, K629G, and K629M based on homology modeling show a loss of favorable interaction between the phosphate group of ATP and the side chain from lysine residue. C) Low levels of ABCB6 ${ }^{\mathrm{K} 629 \mathrm{G}}$ bind to ATP-agarose at $37^{\circ} \mathrm{C}$. Cell lysates were prepared as described above and incubated with ATP-agarose at $37^{\circ} \mathrm{C}$. D) Substitution of lysine residue disrupts ATP binding in ABCB6. Various mutants were tested for ATP binding. 
A

Total lysate

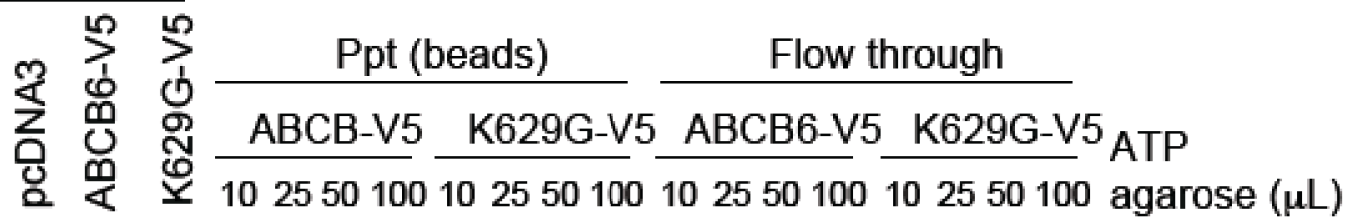
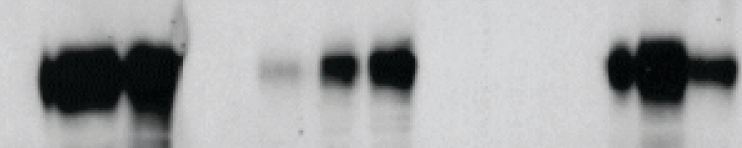

Blot: V5

B

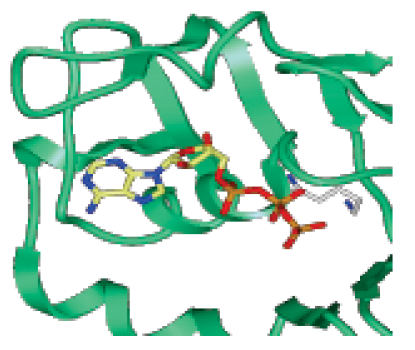

hABCB6_ATP

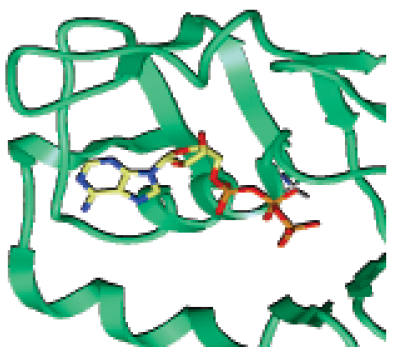

hABCB6_(KtoG)_ATP

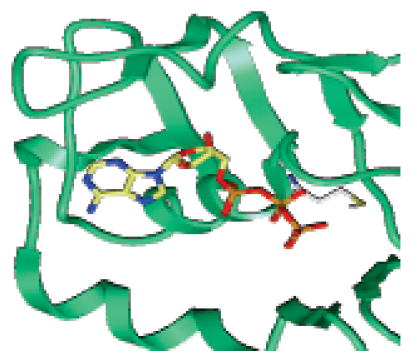

hABCB6_(KtoM)_ATP 
C

Total lysate

Ppt (beads)

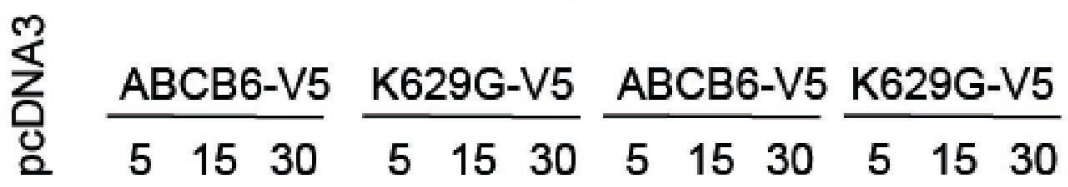

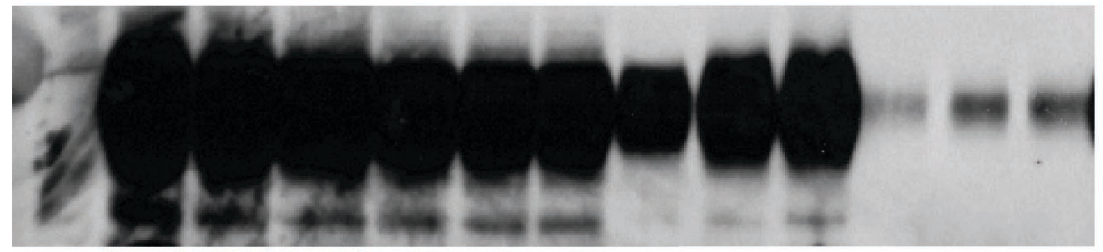

D

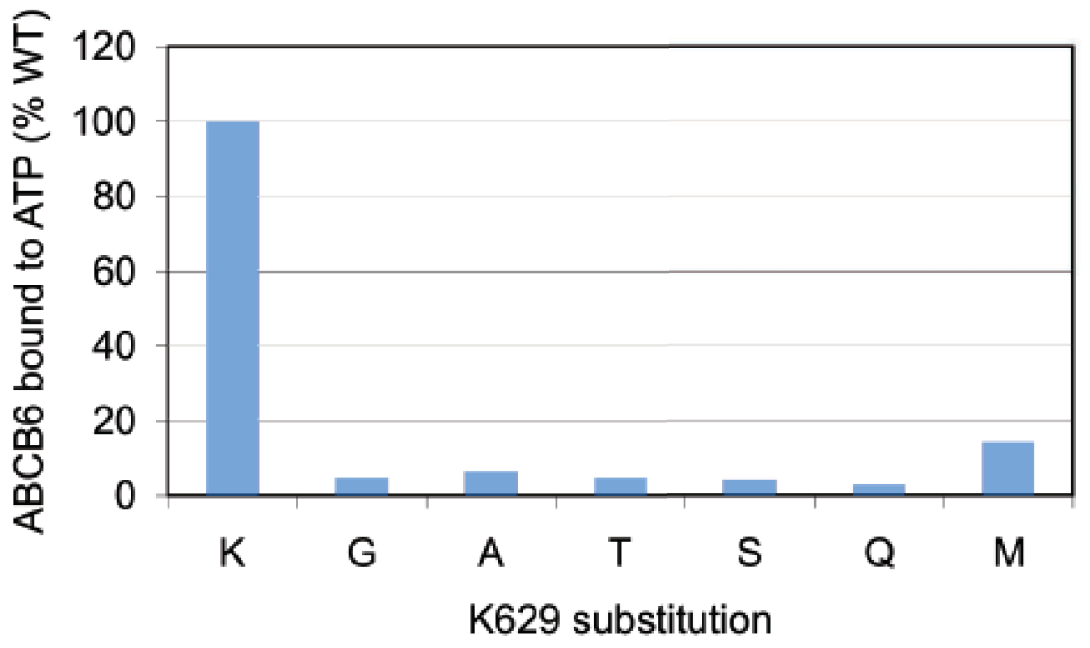

Figure 3.2. (continued). 
ABCB6, it should dissociate from hemin-agarose upon binding to ATP. On the other hand, if the ATP hydrolysis is required for the conformational change, then the incubation with a non-hydrolyzable ATP analog, AMPPNP, will not release ABCB6 from hemin-agarose. Cell lysate was incubated with hemin-agarose and the resultant complex was purified. Various amounts of ATP were added to the ABCB6-heminagarose complex at $24^{\circ} \mathrm{C}$ and the amount of $\mathrm{ABCB} 6$ that remained bound to heminagarose was determined by immunoblotting (Fig. 3.3.A). Forty-percent of ABCB6 dissociated from hemin-agarose in the presence of $50 \mu \mathrm{M}$ ATP, whereas $\mathrm{ABCB} 6^{\mathrm{K} 629 \mathrm{G}}$, which has much lower affinity for ATP, shows no decrease in hemin-agarose binding. At lower ATP concentrations, $\mathrm{ABCB} 6^{\mathrm{K} 629 \mathrm{G}}$ shows a slight increase in hemin-binding, which is not observed with $\mathrm{ABCB} 6$ wild type, for unknown reasons. Nevertheless, $\mathrm{ABCB} 6^{\mathrm{K} 629 \mathrm{G}}$ dissociation from hemin-agarose is minimal even at the highest ATP concentration tested $(0.5 \mathrm{mM},<20 \%)$ in contrast to the wild type ABCB6, in which more than $60 \%$ of ABCB6 dissociated from the beads.

At $24^{\circ} \mathrm{C}$, the rate of ATP hydrolysis is not at its maximum, but to exclude the possibility of ATP hydrolysis playing a role in the dissociation of ABCB6 from heminagarose, AMPPNP, a non-hydrolyzable ATP analog, was used (Fig. 3.3.B, C). Upon the addition of AMPPNP to ABCB6-hemin-agarose complex, the ABCB6 dissociated from hemin-agarose, whereas it had an opposite effect on $\mathrm{ABCB} 6^{\mathrm{K} 629 \mathrm{G}}$. Compared to ATP, higher concentration of AMPPNP was required to induce dissociation of ABCB6 from hemin-agarose. However, it is in good agreement with previous studies that showed 4 mM AMPPNP was required for binding to MRP1 [227].

\subsection{Discussion}

The nucleotide binding to ABCB6 prompted release of ABCB6 from heminagarose. The change of a high affinity state to a low affinity state for hemin-agarose does not require ATP hydrolysis because a non-hydrolyzable ATP analog, AMPPNP, effectively disrupts the interaction between ABCB6 and hemin-agarose. Our results here are consistent with a substrate transport model where the formation of NBD dimer upon substrate and nucleotide binding provide the power stroke for transport. Moreover, unlike another mitochondrial half transporter, Atm1p, a mutation in the conserved lysine in the Walker A domain did not affect homodimerization of ABCB6. The mutation, however, drastically impaired the ATP binding to ABCB6. Therefore, nucleotides did not have effects on the Walker A mutant ABCB6 bound to hemin-agarose.

The mutation of the conserved lysine residue in Walker A motif has different effects depending on $\mathrm{ABC}$ transporters [213]. This can be partly attributed to the asymmetrical NBDs in many full transporters and the heterodimeric half transporters, where one site shows a low affinity for ATP or low ATPase activity. In the asymmetrical NBDs, one ATPase site contains the consensus sequence whereas the other site contains a degenerate sequence [111]. Therefore, the mutation in the degenerate site may not result in a lack of transport activity, because the consensus site provides all the functionalities (e.g., ATP binding and hydrolysis). Because the Walker A mutant ABCB6 
Figure 3.3. Binding of nucleotides to $\mathrm{ABCB} 6$ changes affinity for hemin-agarose. A) A schematic drawing of roles ATP binding and ATP hydrolysis can play in substrate transport cycle. B) ATP induces a conformational change from a high affinity substrate binding to a low affinity binding state. C) AMPPNP induces a dissociation of ABCB6 from hemin-agarose. 
A

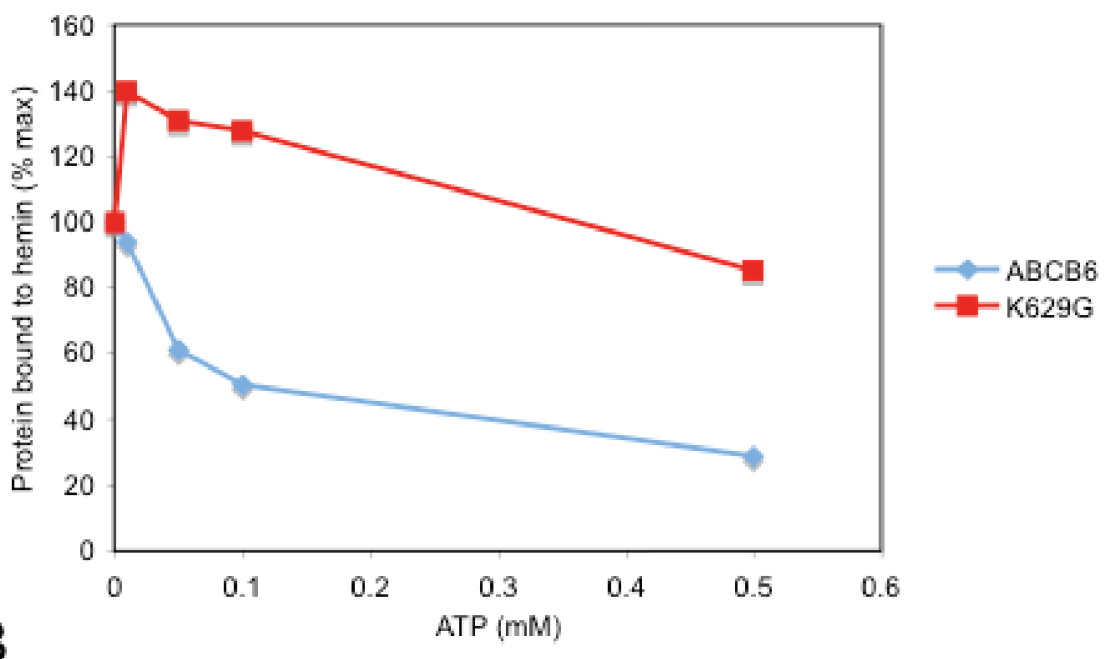

B
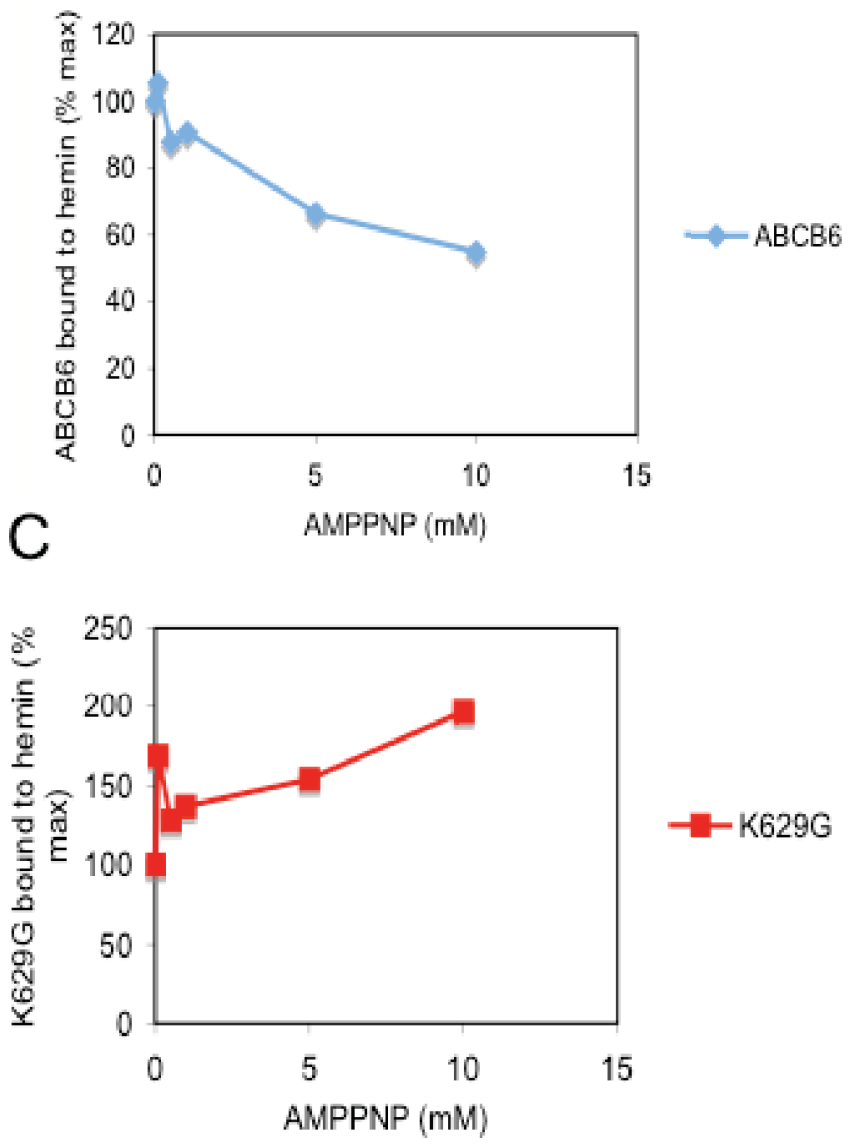
homodimerizes, both ATPase sites contain mutated Walker motif and, therefore, ATP binding is severely impaired. Whether ABCB6 is normally bound to ATP or ADP under physiological conditions, which was suggested for TAP1/TAP2 heterodimer [111], still needs to be determined. In TAP1/TAP2, substrate binding promotes ATP exchange at the NBD, which results in the formation of NBD dimer. The substrate binding is unlikely to be affected by the presence of ATP because ATP depletion does not affect peptide binding to TAP1/TAP2 [228]. In addition, inability to bind ATP did not affect the Walker A mutant ABCB6 binding to hemin-agarose. In either case, ATP binding affects the dimer formation of NBD $[110,111,224]$. Purified TAP1/TAP2 NBDs do not heterodimerize, suggesting that the formation of dimer is not an intrinsic feature [111].

The equilibrium binding to hemin-agarose saturated at $1 \mathrm{mM}$ and a slight decrease in the binding was observed when higher concentrations of hemin-agarose $(>3 \mathrm{mM})$ were added. The concentration of hemin-agarose needed to reach the saturation was relatively high. However, we have previously shown that $40 \mu \mathrm{M}$ hemin displaces $>90 \%$ of ABCB6 bound to hemin-agarose [204] suggesting that the $\mathrm{K}_{\mathrm{d}}$ observed using hemin-agarose is likely to be much higher than the actual $\mathrm{K}_{\mathrm{d}}$ for free hemin. The agarose beads may be a limiting factor for the amount of $\mathrm{ABCB} 6$ molecule to bind to hemin-agarose requiring apparently much higher concentration to reach a saturation point.

Even though functions of NBD and MSD are tightly coupled, how the changes in NBD are transmitted to MSD and vice versa has not been shown clearly because the structural data of a mammalian ABC transporter are lacking. Structural data on the soluble NBDs provided an insight into how ATPase sites are formed between two NBDs and the conformational changes that they undergo when bound to nucleotides $[110,111]$. The structure of a sandwich NBD dimer and other biochemical data led to a substrate transport model that ATP binding induces a conformational change sufficient to release substrates [114]. The structure of nucleotide-bound bacterial ABC transporter Sav1866, which shows a significant sequence similarity to human $\mathrm{ABCB}$ subfamily members, shows an outward opening of TM helices [97]. The NBD is in a closed dimer and the cavity in the membrane created by TM helices is not accessible from the cytosol. Therefore, the cavity is suggested to represent the substrate translocation path into the extracellular space. The outward facing cavity of ATP-bound MDR1 has also been shown using electron microscopy [229, 230]. Binding of AMPPNP to ABCB6 results in a release of $\mathrm{ABCB} 6$ from hemin-agarose suggesting that $\mathrm{ABCB} 6$ undergoes conformational changes between a high affinity to a low affinity state for substrates depending on nucleotide binding status. A higher concentration of AMPPNP was required to release $50 \%$ of $\mathrm{ABCB} 6$ from hemin-agarose compared to ATP. However, the concentration used in this study $(5 \mathrm{mM})$ to observe an effect is consistent with previous studies that showed $4 \mathrm{mM}$ AMPPNP was required to bind to MRP1 NBD1 [227]. The condition this experiment was performed, room temperature, is not ideal for ATP hydrolysis and, therefore, the effect of ATP hydrolysis on the conformational changes should be minimal. Taken together, our results provide biochemical evidence that a substrate is released from $\mathrm{ABC}$ transporter upon nucleotide binding and support the ATP switch model. 


\section{CHAPTER 4. ABCB6 CONTAINS AN N-TERMINAL ER EXIT SIGNAL THAT IS CONSERVED IN OTHER ABC TRANSPORTERS}

\subsection{Introduction}

ATP-binding cassette $(\mathrm{ABC})$ transporters are involved in the ATP-dependent transmembrane movement of various compounds [2]. Their diverse physiological functions have been revealed because ABC transporter deficiencies lead to human diseases such as cystic fibrosis, Dubin-Johnson syndrome, X-linked sideroblastic anemia and ataxia and hyperinsulinemic hypoglycemia [2]. ABC transporters at the plasma membrane extrude endogenous compounds, but also protect cells by reducing the accumulation of toxic compounds. Moreover, ABC transporters are found in subcellular organelles and play key roles in biological processes such as heme biosynthesis, [Fe-S] cluster formation, and antigen presentation.

$\mathrm{ABC}$ transporter localization specifically to the plasma membrane or to subcellular organelles suggests inherent signals within the transporter are required to ensure proper localization. However, among ABC transporters, it is difficult to identify a typical sequence or modification that affects all membrane transporters. For example, lack of ABCG8 glycosylation causes ER retention, and apical localization of ABCB11 requires at least two out of the four $\mathrm{N}$-glycosylation sites [133, 231]. In contrast, CFTR/ABCC7 membrane localization is independent of N-glyosylation [232].

In the ER, several covalent post-translational modifications occur, which include signal sequence cleavage, N-glycosylation, and disulfide bond formation. Disulfide bonds are formed in the ER via the action of protein disulfide isomerase enzymes. If essential disulfide bond(s) are not formed, some proteins are retained in the ER [233] by a process referred to as "thiol retention" [234]. Thiol retention has been shown to regulate the ER exit of adiponectin [235] and IgM. If the free thiols in IgM are not oxidized by formation of disulfide bonds or by other means, then IgM does exit the ER [236, 237]. IgM egress from the ER can be manipulated by changing the cellular redox environment with thiol reductants (e.g. DTT or $\beta$-ME) [238].

We have previously shown that ABCB6 is a homodimeric porphyrin transporter, which through its localization at the mitochondrial outer membrane facilitates heme biosynthesis [204]. ABCB6's high expression in human and murine fetal liver coincides with definitive erythropoiesis. As expected, ABCB6 is upregulated during differentiation of progenitor cells toward the erythroid lineage. This is consistent with the requirement for porphyrins in erythropoiesis as erythroid cells require a high heme production to ensure hemoglobin synthesis [176]. Furthermore, ABCB6 function is necessary to ensure efficient heme biosynthesis. Heme biosynthesis occurs in two compartments, and the energetic constraints of shuttling the anionic heme precursor, CPgenIII from the cytosol into the intermembrane space where the next enzyme in the pathway resides, make this a potential rate-limiting step that ABCB6 can overcome by transporting CPgenIII across the outer membrane [176]. 
While ABCB6 is a mitochondrial outer membrane protein, it is not clear how ABCB6 trafficks in the cells, what signals are required for the trafficking, or if these signals are required for function. In this study, we show that ABCB6 is modified by glycan addition at only one site. Moreover, ABCB6 trafficks through the ER to the Golgi, a process that requires the formation of a disulfide bond between two cysteines present at the N-terminus. Notably the ABCC subfamily members that insert their N-terminal into the ER lumen (e.g. ABCC1, C2, etc.) contain N-terminal cysteines that may form a disulfide bond. The functional significance of these cysteines were revealed in SUR1/ABCC8, a plasma membrane $\mathrm{K}+$ channel regulator [83]. We discovered a single mutation in this conserved cysteine in SUR1/ABCC8 from a patient with defective $\mathrm{K}+$ channel function. As a consequence of this single point mutation in the conserved cysteines, SUR1/ABCC8 was incapable of trafficking to the plasma membrane. Our findings have uncovered a conserved $\mathrm{N}$-terminal disulfide bond, which provides an important signal for ER exit of $\mathrm{ABC}$ transporters and suggests that alterations in the redox status of the ER can impact $\mathrm{ABC}$ transporter trafficking.

\subsection{Experimental Procedures}

\subsubsection{Cell Culture}

NIH3T3 cells, Mel cells and K562 vector or ABCB6-Flag cells were cultured in Dulbecco's modified Eagle's medium (DMEM) containing 4500 mg/L glucose supplemented with 10\% FCS (HyClone, Logan, UT), 2 mM L-glutamine, 100 units $/ \mathrm{ml}$ penicillin, and $100 \mathrm{ug} / \mathrm{ml}$ streptomycin (Gibco, Carlsbad, CA) in humidified atmosphere containing $5 \% \mathrm{CO}_{2}$ at $37{ }^{\circ} \mathrm{C}$. NIH3T3 cells were seeded on 6 well-clustered dishes $(3 \mathrm{x}$ $10^{5}$ cells/well) 24 hours prior to transfection, and then transiently transfected with expression plasmids (total of $1 \mu \mathrm{g}$ except for when otherwise indicated) using LipofectAMINE Plus (Invitrogen, Carlsbad, CA) according to the manufacturer's protocols.

\subsubsection{Site-Directed Mutagenesis}

Plasmids pcDNA3-hABCB6-Flag and pcDNA3.1-hABCB6-V5-His were designed to express human ABCB6 with a C-terminal tag, Flag or V5 respectively as previously described [204]. A point mutation was introduced into pcDNA3.1-hABCB6V5-His using a Quikchange site-directed mutagenesis kit (Stratagene, La Jolla, CA) and primers (N447Q, sense, 5'-CGTGCTATGAACACACAGGAGCAAGCTACCCGGGCACGAGCAGTG-3', antisense, 5' - CACTGCTCGTGCCCGGGTAGCTTGCTCCTGTGTGTTCATAGCACG-3'; N498Q, sense, 5'-GCGCTTCACTGGTTTTACTACAACAGACCCAGAACCTGGTGATTG3', antisense, 5'-CAATCACCAGGTTCTGGGTCTGTTGTAGTAAAACCAGTGAAGCGC-3'; N677Q, sense, 5'-CCCAAGACACTGTCCTCTTTCAAGACACCATCGCCGACAATATC-3', antisense, 5'-GATATTGTCGGCGATGGTGTCTTGAAAGAGGACAGTGTCTTGGG-3'; N775Q, sense, 5'-TCTGGCCAA- 
AGTCTGTGCCCAACGCACCACCATCGTAGTGGC-3', antisense, 5'-GCCACTACGATGGTGGTGCGTTGGGCACAGACTTTGGCCAGAG-3'; N6Q, sense, 5'-CTTGCCATGGTGACTGTGGGCCAATACTGCGAGGCCGAAGGGCC-3', antisense, 5'-GGCCCTTCGGCCTCGCAGTATTGGCCCACAGTCACCATGGCAAG-3'; C8S, sense, 5'GGTGACTGTGGGCAACTACTCCGAGGCCGAAGGGCCCGTGG-3', antisense, 5'CCAC-GGGCCCTTCGGCCTCGGAGTAGTTGCCCACAGTCACC-3'; C26A, sense, 5'-GCAGGATGGCCTGAGTCCCGCCTTCTTCTTCACGCTCGTGCC-3', antisense, 5'-GGCACGAGCGTGAAGAAGAAGGCGGGACTCAGGCCATCCTG-C-3'; C50A, sense, 5'-GCCTTGGTGCTGGCTCTTCCCGCTAGACGCCGGGAGCGG-CCCGC-3', antisense, 5'-GCGGGCCGCTCCCGGCGTCTAGCGGGAAGAGCCAGCACCAAGGC-3'; C120A, sense, 5'-CTGGAGAGTCTGGCCGGCGCCGCTGGCCTGTGGCTGCTTGTCGTG-3', antisense, 5'-CACGACAAGCAGCCACAGGCCAGCGGCGCCGGCCAGACTCTCC-AG-3'). All genes were thoroughly sequenced after mutagenesis. A point mutation was introduced into pcDNA3.1-hABCB6-N447Q-V5-His at N498 as described above to yield pcDNA3.1-hABCB6-N447,498Q-V5-His. The pcDNA3.1-hABCB6-Q4V5-His mutant was generated by introducing a point mutation into pcDNA3.1-hABCB6N447, 498Q-V5-His at N677 and then at N775. An additional mutation was introduced into pcDNA3.1-hABCB6-Q4-V5-His at N6 to generate pcDNA3.1-hABCB6-Q5-V5-His. pcDNA3.1-hABCB6-C8S,C26A-V5-His was generated by introducing a point mutation into pcDNA3.1-hABCB6-C8S-V5-His at C26.

To generate truncated mutant constructs to determine the disulfide bond formation, point mutations were introduced into constructs pcDNA3.1-hABCB6-V5 at $\mathrm{C} 50$ and then at $\mathrm{C} 120$ using primers described above. And then the N-terminus of the mutant ABCB6 construct containing amino acids 1 to 210 with Flag tag at the C-terminus was amplified using primers (sense, 5'-GCCATGGTGACTGTGGGCAACTACTGCGAGGCCG-3', and antisense, 5'-CCTACTTATCGTCGTCATCCTTGTAATCACGAAGTCCAGGGGCCCAGAG-3') with the following condition $\left(94^{\circ} \mathrm{C}, 4 \mathrm{~min}\right.$, denaturing at $94^{\circ} \mathrm{C}$ for $30 \mathrm{sec}$, annealing at $56^{\circ} \mathrm{C}$ for $30 \mathrm{sec}$, and reaction at $68^{\circ} \mathrm{C}$ for $1 \mathrm{~min}$ for 30 cycles). The PCR product was purified from agarose gel and TA-cloned into pCR2.1TOPO vector (Invitrogen). The inserts were sublconed into pcDNA3 using HindIII/XbaI sites to obtain pcDNA3-hABCB6-C50/120A-N1-210-Flag.

\subsubsection{Immunoblotting}

Twenty-four hours after transfection, cells were washed with PBS and then scraped into $1 \mathrm{ml}$ cold PBS containing $1 \mathrm{x}$ commercial protease inhibitor cocktail (complete EDTA free, Roche, Mannheim, Germany). Cells were pelleted by centrifugation at $1,000 \mathrm{x}$ g for 4 min at $4^{\circ} \mathrm{C}$, and were resuspended in buffer $\mathrm{A}(50 \mathrm{mM}$ Tris- $\mathrm{HCl}$ ( $\mathrm{pH} 7.5), 150 \mathrm{mM} \mathrm{NaCl}, 10 \%$ glycerol, and $1 \mathrm{x}$ commercial protease inhibitor cocktail (complete, Roche)), and sonicated (three $10 \mathrm{sec}$ pulses). Nonidet P40 (NP-40, final concentration of $1 \%$ ) was added to solubilize the cells, followed by constant rocking for $60 \mathrm{~min}$ at $4^{\circ} \mathrm{C}$. Samples were then centrifuged at $17,000 \mathrm{x}$ g for $15 \mathrm{~min}$ at $4^{\circ} \mathrm{C}$ to remove cell debris. Protein concentration in the supernatant was measured by Bradford method (Protein Assay, Bio-Rad Laboratories Inc., Hercules, CA). The lysate was further 
processed for either immunoblotting or glycosidase digestion. For immunoblotting, Laemmli sample buffer containing $\beta$-ME unless otherwise indicated (final concentration $1 \mathrm{x}$, Bio-Rad Laboratories Inc.) was added to the supernatant and the samples were fractionated on $7.5,10$ or $12.5 \%$ SDS-PAGE. Afterward, proteins were transferred to a nitrocellulose membrane (Amersham Biosiences). The blots were blocked with 10\% skim milk in PBS-T and incubated with either anti-FLAG (M2 or polyclonal, Sigma), anti-V5 polyclonal (MBL, Nagoya, Japan), anti-ABCB6 (serum), or anti-ABCB6 monolconal antibodies. The blots were washed with PBS-T, incubated with secondary antibodies (horseradish peroxidase-conjugated anti-rabbit or anti-mouse IgG F(ab') fragment, Amersham Pharmacia Biotech., Liscataway, NJ), and immunoreactive proteins detected by using enhanced chemiluminescence (ECL, Amersham Pharmacia Biotech.).

\subsubsection{Glycosidase Digestion}

Lysates were prepared as described above from NIH3T3, K562, or Mel cells. After denaturation in $1 \mathrm{x}$ denaturing buffer (0.5\% SDS, $1 \% \beta$-marcaptoehtanol), the samples were incubated at $37^{\circ} \mathrm{C}$ for $1 \mathrm{hr}$ with or without PNGase $\mathrm{F}$ (New England Biolabs, Inc., Beverly, MA) in reaction buffer (50 mM Na $2 \mathrm{PO}_{4}(\mathrm{pH} 7.5)$ and 1\% NP-40) according to the manufacturer's recommended procedures. For EndoH digestion, the denatured samples were incubated with or without the enzyme in $50 \mathrm{mM}$ sodium citrate buffer.

\subsubsection{Mitochondria Isolation}

K562 or Mel cells were pelleted, washed with PBS, and resuspended in resuspension buffer (10 $\mathrm{mM} \mathrm{NaCl}, 1.5 \mathrm{mM} \mathrm{MgCl}_{2}, 10 \mathrm{mM}$ Tris- $\mathrm{HCl}(\mathrm{pH} 7.6)$, and $1 \mathrm{x}$ protease inhibitor cocktail (Roche). After 10 min incubation on ice to allow the cells to swell, they were broken with type B Dounce homogenizer. $2.5 \mathrm{x}$ membrane stabilizing buffer (525 mM mannitol, $175 \mathrm{mM}$ sucrose, $12.5 \mathrm{mM}$ Tris- $\mathrm{HCl}$ (pH 7.6), and $2.5 \mathrm{mM}$ EDTA) was added to the homogenate to a final concentration of $1 \mathrm{x}$. The samples were centrifuged at $1,300 \mathrm{x}$ g for $5 \mathrm{~min}$ at $4{ }^{\circ} \mathrm{C}$ to remove cell debris and nuclei. The supernatant was further centrifuged at $17,000 \mathrm{x}$ g for $15 \mathrm{~min}$ at $4^{\circ} \mathrm{C}$ to pellet mitochondria.

\subsubsection{Pulse Chase}

K562 vector or ABCB6-Flag cells $\left(\sim 2 \times 10^{6}\right.$ cells) were washed with warm $1 \mathrm{x}$ Hank's buffer and incubated in labeling media (DMEM without L-methionine, L-cysteine (Cellgro, Manassas, VA), 10\% dialyzed FBS (Hyclone), 2 mM L-glutamine) containing $0.1 \mathrm{mCi} / \mathrm{ml}[35 \mathrm{~S}]$ (Trans ${ }^{35} \mathrm{~S}-$ label ${ }^{\mathrm{TM}},\left[{ }^{35} \mathrm{~S}\right]$ L-Methionine, $\left[{ }^{35} \mathrm{~S}\right]$ L-Cysteine, 1175 $\mathrm{Ci} / \mathrm{mmol}$, MP Biomedicals, Inc. Irvine, CA) for $20 \mathrm{~min}$ at $37^{\circ} \mathrm{C}$. Cells were then washed once with chase media (complete DMEM supplemented with $2 \mathrm{mM}$ L-methionine and Lcysteine) and chased for times indicated in the figures. At the end of each chase time interval, cells were washed with cold PBS, pelleted, flash frozen in liquid $\mathrm{N}_{2}$ and stored 
at $-80^{\circ} \mathrm{C}$ until use. Cells were lysed in NP-40 lysis buffer ( $50 \mathrm{mM}$ Tris- $\mathrm{HCl}(\mathrm{pH} 7.5), 150$ $\mathrm{mM} \mathrm{NaCl}, 0.5 \%$ deoxycholate, and $0.5 \% \mathrm{NP}-40$ ) for $15 \mathrm{~min}$ on ice and cell debris was removed by centrifugation at $15,000 \mathrm{x}$ g for $10 \mathrm{~min}$ at $4{ }^{\circ} \mathrm{C}$. The lysate was incubated with $20 \mu \mathrm{l}$ anti-Flag M2 antibody conjugated agarose (50\% slurry, Sigma) for $2 \mathrm{hr}$ at $4^{\circ} \mathrm{C}$ with constant rotation. The beads were washed three times with Washing buffer $(50 \mathrm{mM}$ Tris$\mathrm{HCl}$ (pH 7.5), $400 \mathrm{mM} \mathrm{NaCl}, 0.5 \%$ deoxycholate, $0.5 \% \mathrm{NP}-40$ ) and then resuspended in $2 \times$ Laemmli SDS sample buffer containing $\beta$-ME. Samples were separated by SDSPAGE. The gel was fixed, stained by Coomassie G-250 (GelCode Blue, Pierce), incubated with Amplify ${ }^{\mathrm{TM}}$ fluorographic reagent (Amersham, GE Healthcare) and was dried. $\left[{ }^{35} \mathrm{~S}\right]$-labeled proteins were detected by autoradiography.

\subsubsection{Glycosylation Site Prediction}

To search for potential N-glycosylation site(s) in ABCB6 sequence, PROSITE motif search, which scans for a consensus motif $\mathrm{N}-\mathrm{X}-\mathrm{S} / \mathrm{T}-\mathrm{X}$ where $\mathrm{X}$ can be any amino acid except for proline, was performed (http://www.predictprotein.org, accessed Oct. 13, 2004).

\subsubsection{Detection of SUR1}

A series of SUR1/ABCC8 mutant constructs were generated where Cys6 and Cys 26 were singly or doubly mutated to alanine or serine. Rest of the 27 cysteine residues were mutated to either alanine or serine in these constructs. COSm6 cells were co-transfected with the various SUR1 constructs with KIR6.2. SUR1 was affinity labeled with $\left[{ }^{125} \mathrm{I}\right]$-azidoglibenclamide, fractionated on SDS-PAGE, and detected by autoradiography.

\subsection{Results}

\subsubsection{Both Endogenous and Exogenous ABCB6 Are Glycosylated}

Glycosylated proteins, which are synthesized in the ER, are often observed as broad or smeared bands on immunoblotting. Although ABCB6 is a mitochondrial protein, which are typically not glycosylated, we tested if it was modified by N-linked glycans because ABCB6 appeared as a broad band on immunoblots. Crude mitochondrial preparations from either human erythroleukemia K562 or murine erythroleukemia Mel cells were treated with either PNGase F to remove high mannose and complex glycans from asparagine residues or Endo H, which only reacts with high mannose glycans found on proteins as long as they remain in the ER. Immunoblot analysis (using both an antiABCB6 antiserum and a monoclonal antibody we developed to a conserved epitope) revealed faster migration of both human and murine ABCB6 after PNGase F but not Endo $\mathrm{H}$ treatment (Fig. 4.1.). The PNGase F sensitivity and Endo H resistance 
Figure 4.1. ABCB6 is glycosylated.

Crude mitochodria preparations from A) K562, human erythroleukemia or B) Mel, murine erythroleukemia cells were treated with PNGase F or Endo H and ABCB6 was detected by immunoblotting. Cell lysates from C) K562 cells expressing vector or ABCB6-Flag or D) NIH3T3 cells transiently transfected with ABCB6-V5 were treated with PNGase F and analyzed by immunoblotting. E) ABCB6 was no longer glycosylated when K562 cells expressing ABCB6-V5 were treated with tunicamycin. F) Pulse chase assay using K562 cells expressing ABCB6-Flag showed the glycosylated ABCB6 at 60 $\min$. 


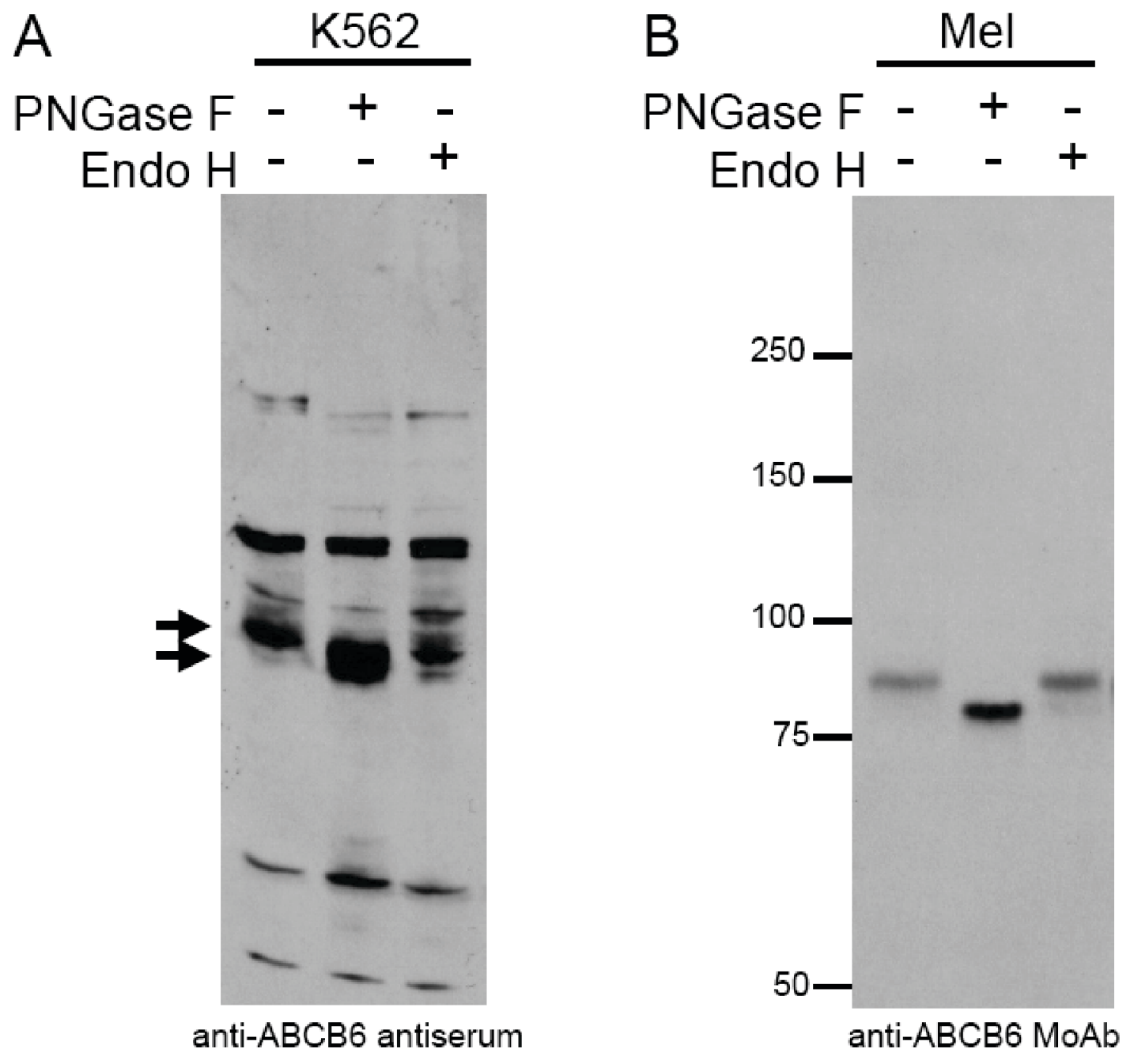



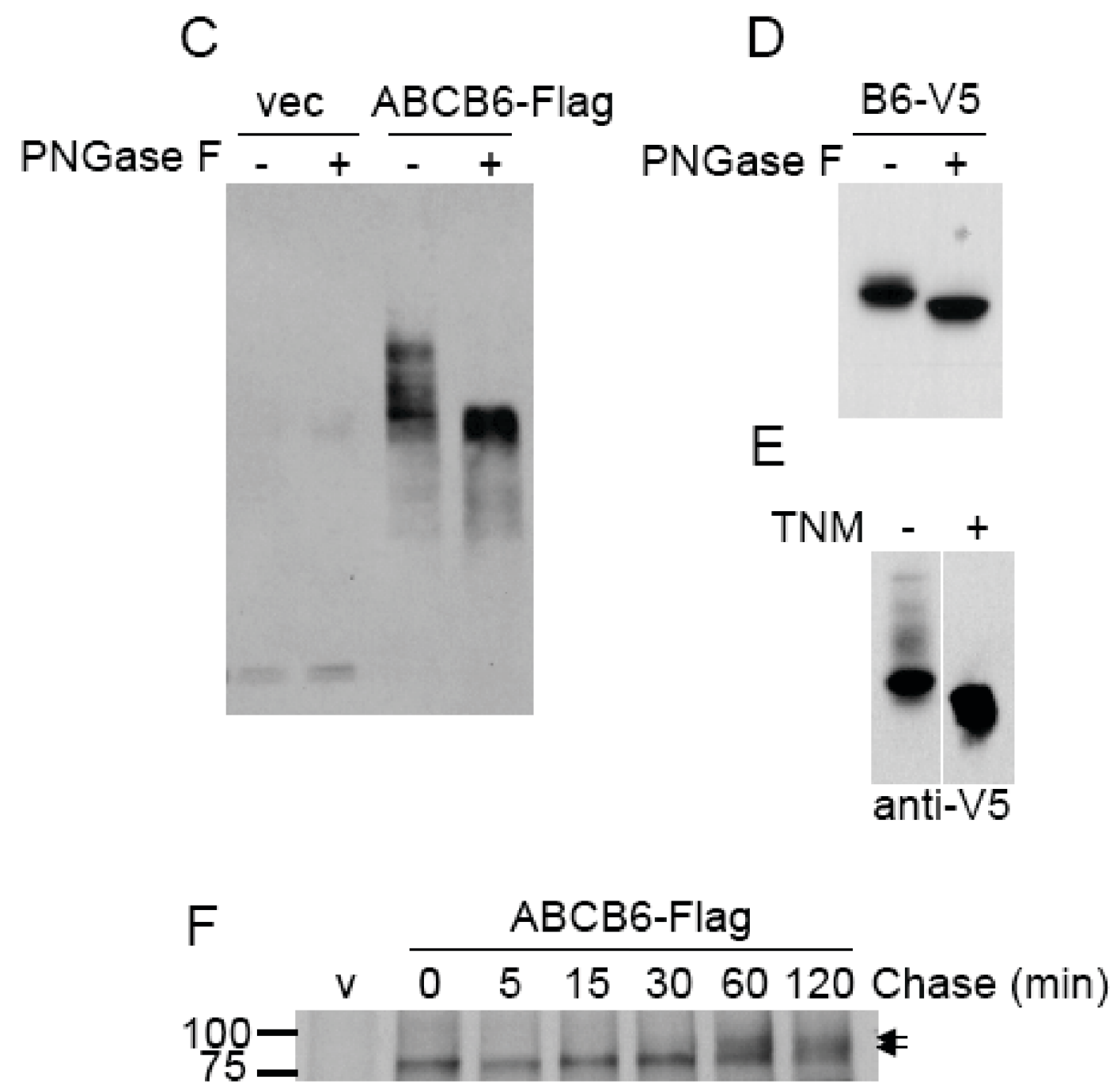

Figure 4.1. (continued). 
demonstrate that both human and murine ABCB6 are glycosylated and traffick through the ER to the Golgi.

We next tested whether epitope-tagged human ABCB6 exhibited the same pattern of glycosidase sensitivity. We found that the ABCB6, transfected into K562 and NIH3T3 cells, was PNGase F sensitive (Fig. 4.1.). These results demonstrate that epitope-tagged ABCB6 trafficks like endogenous ABCB6. Moreover, this processing pathway is indistinguishable between erythroid and non-erythroid cells. The extent of glycosylation does differ between species with ABCB6 in human cells showing multiple bands whereas ABCB6 in mouse cells shows only one discrete band. To understand the pathway leading to ABCB6 glycosylation, we used two experimental approaches: pulse chase labelling and treatment with tunicamycin (TNM), an inhibitor of glycan synthesis in the ER. Treatment of K562 cells stably expressing ABCB6-V5 with TNM abolished the high molecular weight bands (Fig. 4.1.). Tunicamycin sensitivity of ABCB6 glycan modification confirms that ABCB6 trafficks through the ER. We performed a pulse chase experiment to follow the maturation of $\mathrm{ABCB}$. The $\mathrm{CHO}-\mathrm{TRex}$ system was used previously to transiently express $\mathrm{ABCB} 6$ in order to demonstrate $\mathrm{ABCB} 6$-activated de novo porphyrin biosynthesis [204]. Therefore, given that the exogenous ABCB6 activates heme biosyntheis like the endogenous protein, exogenous ABCB6 was likely to proceed via a processing pathway similar to the endogenous one in this system. CHO cells harboring the reverse tet operator were transfected with pcDNA4-ABCB6-Flag, treated with tetracycline, and then pulsed for $20 \mathrm{~min}$ with $\left[{ }^{35} \mathrm{~S}\right]-\mathrm{Cys} / \mathrm{Met}$ labeling mixture in media lacking Cys/Met. Cells were collected after various chase intervals, and immunoprecipitated $\left[{ }^{35} \mathrm{~S}\right]$-labeled ABCB6 was separated by SDS-PAGE and detected by autoradiography. Slower migrating forms of ABCB6 were detected at 60 and 120 min after the pulse, which are likely to correspond to further modification of the N-linked glycans in the Golgi (Fig. 4.1.).

\subsubsection{ABCB6 Is Glycosylated at an Atypical $N-X-C$ Site}

Potential N-glycosylation site(s) in ABCB6 sequence were identified using PROSITE motif search (http://www.predictprotein.org, [239]), which scans a consensus glycosylation motif N-X-S/T-X (X can be any amino acid except for proline [240]). The search results revealed four potential glycosylation sites in human ABCB6, which are also conserved in mouse Abcb6 (Fig. 4.2.A). We have previously shown that the Cterminus of ABCB6 (containing the nucleotide binding domain) resides in the cytoplasm [204]; therefore, Asn677 and Asn775 are unlikely targets for glycosylation because they will not face the site of glycosylation in the ER lumen. Nevertheless, each asparagine (N) residue was individually substituted with glutamine (Q) to develop four ABCB6 mutant constructs. Further, a double asparagine mutant where both Asn447 and Asn498 were substituted with glutamine residues was generated in the event more than one

glycosylation site is used. All five ABCB6 asparagine mutants contained a V5 epitope tag at the $\mathrm{C}$-terminus and were transiently expressed in NIH3T3 cells. The migration patterns of ABCB6 asparagine mutants were assessed after PNGase F treatment (Fig. 4.2.B). Unexpectedly, individual or double $\mathrm{ABCB} 6$ asparagine mutants showed the same 
Figure 4.2. ABCB6 is glycosylated only at the atypical $\mathrm{N}-\mathrm{X}-\mathrm{C}$ site.

A) Four consensus and one atypical glycosylation sites in human ABCB6. B, C) Asparagine residues in the consensus or the atypical (N6Q) sites were substituted with glutamine residues. NIH3T3 cells were transiently transfected with B) single mutants and C) Q4 mutant in which all four Asn residues were mutated, or D) single mutant N6Q or Q5 in which all five Asn residues were mutated with glutamine. Proteins were subjected to glycosidase treatment and analyzed by immunoblotting using anti-V5 antibody. The presence of glycans was determined by the mobility shift in the immunoblotting. 
A

\section{$\mathrm{N}$-Glycosylation motif: $\mathbf{N}-\mathbf{X}-\mathbf{S} / \mathbf{T}-\mathbf{X}$}

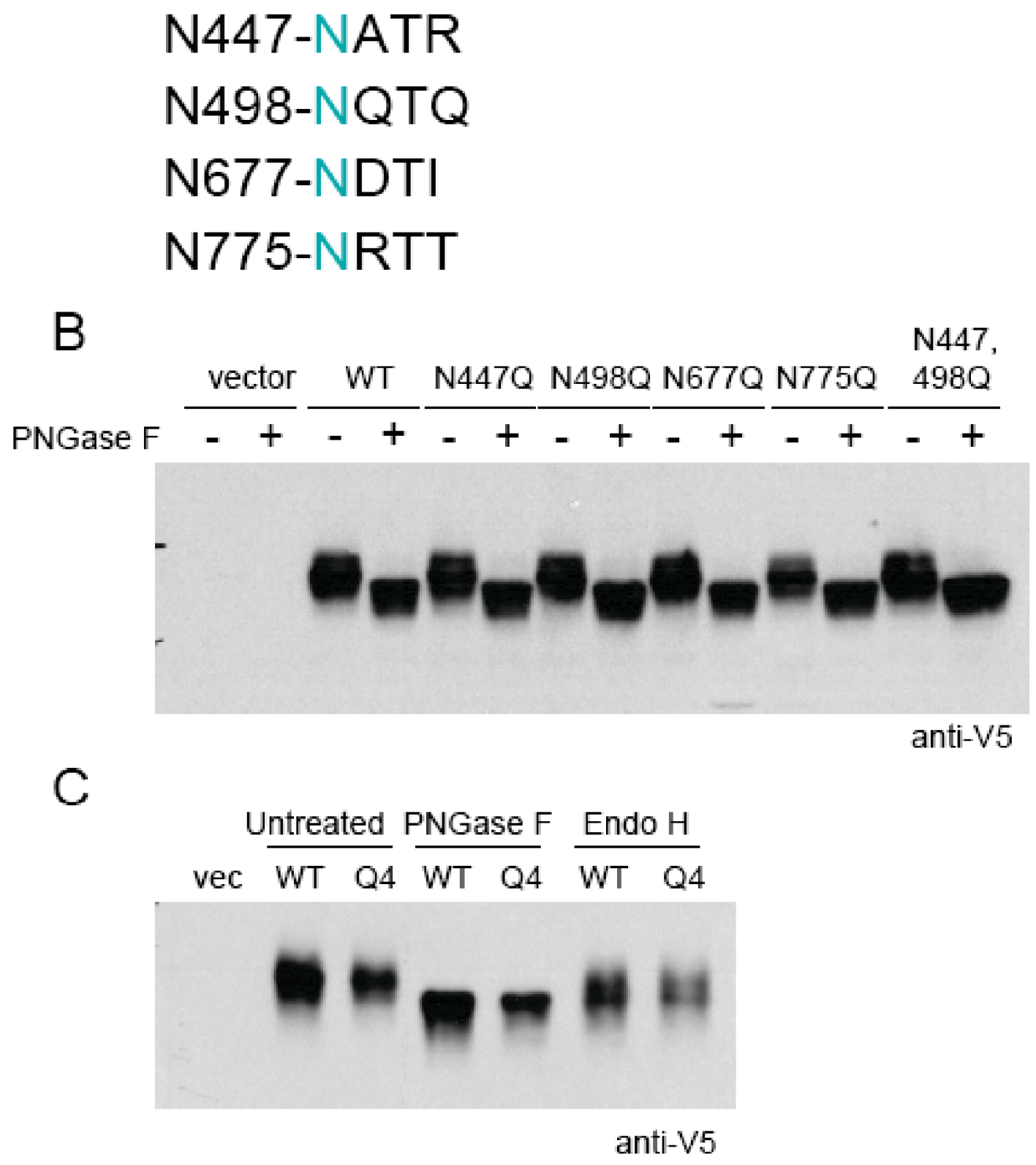


D

Atypical glycosylation motif: $\mathrm{N}-\mathrm{X}-\mathrm{C}$

N6-NYCE

Q4: N447,498,677,775Q

Q5: N6,447,498,677,775Q N6Q

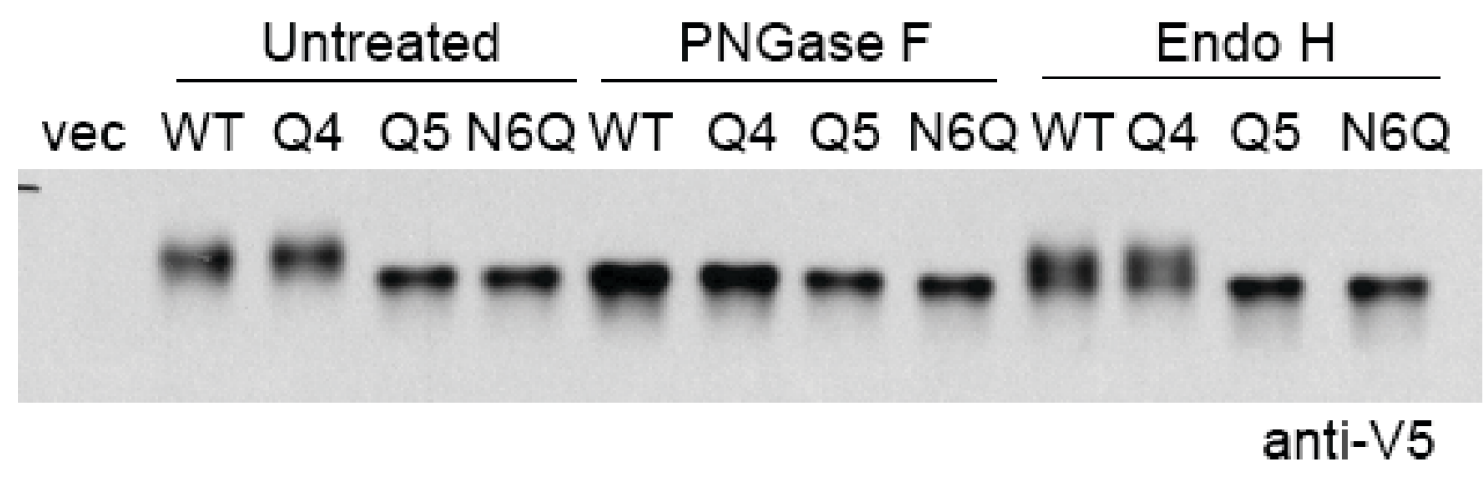

Figure 4.2. (continued) 
migration pattern as the wild-type protein, in that they migrated faster after treatment with PNGase F, which indicates these asparagine mutations do not change ABCB6 glycosylation. Therefore, to rule out the possibility of multi-site glycosylation, all four asparagine residues in the consensus glycosylation motifs were substituted with glutamine residues (designated as Q4 mutant). Surprisingly, Q4 behavior was identical to the wild-type protein such that the faster migrating band (immature ABCB6) was only seen when treated with PNGase F (Fig. 4.2.). These results indicate that other glycosylation site(s) must exist.

An extensive review of the available literature revealed five proteins that use a cysteine residue instead of the typical serine or threonine at the glycosylation site (N-XC) [241-245]. These proteins are typically secreted proteins. However, because these proteins have other typical glycosylation sites and the N-X-C motif represents a minority of the glycosylation sites, its functional significance remains unknown. The five proteins are: Protein C (1/4), von Willebrand factor (1/12), fetal antigen I (2/3), transferrin (1/3), and CD69 (1/2), and the proportion of N-X-C motifs out of the total number of glycosylation sites is indicated in the parentheses. Examination of the ABCB6 sequence revealed one atypical glycosylation motif near the N-terminus, N-Y-C starting at position 6 (Fig. 4.2.). To test the possibility that this site was used in ABCB6, the asparagine residue at position 6 was substituted with a glutamine residue in both wild-type and Q4 constructs to generate N6Q and Q5 ABCB6 constructs respectively. The ABCB6 asparagine mutants were transiently transfected into NIH3T3 cells, treated with PNGase F and analyzed by immunoblotting using anti-V5 antibody (Fig. 4.2.). Both Q5 and N6Q migrated faster than the wild-type ABCB6-V5 and their mobility was unaffected by PNGase F treatment and identical to the PNGase F-treated wild-type protein. These findings indicate that ABCB6 uses the N-X-C motif as its sole glycosylation site.

Generally, ABC half transporters contain six TM helices; however, the unique glycosylation site at the $\mathrm{N}$-terminus and the cytoplasmic localization of the $\mathrm{C}$-terminus allowed us to develop a topological model that contain more than six TM helices for ABCB6. Accordingly, programs such as TopPred and TMHMM predicted ABCB6 to contain nine TM helices [218, 219], while HMMTOP and SOSUI predicted 11 and 10, respectively [220, 246, 247]. TopPred, TMHMM, and HMMTOP predicted that the sole glycosylation site is protruded into the ER lumen. The absence of glycans at Asn677 and Asn775 is consistent with the C-terminus in the cytoplasm, which is in agreement with our previous biochemical findings [204]. These results indicate that $\mathrm{N}$ - and C-termini are on the opposite sides of the membrane and suggest that ABCB6 contains an odd number of TM helices. The lack of glycosylation at the consensus sites Asn447 and Asn498 suggests that these residues are not accessible to the glucosyltransferase (although not all $\mathrm{N}-\mathrm{X}-\mathrm{S} / \mathrm{T}$ sites are used in secretory proteins), and, therefore, are likely to reside in the cytoplasm. Only the predictions from HMMTOP and TMHMM satisfy all these criteria, in which HMMTOP model contains extra two TM helices (248-271A.A. and 392326A.A.) in place of a large cytoplasmic loop in the TMHMM prediction (Fig. 4.3.). It is notable that we have identified a form of ABCB6 that lacks amino acids 240 to 289, which we have previously shown is incapable of binding heme. 
Figure 4.3. ABCB6 is predicted to contain 9 TM helices.

Schematic drawing of membrane topology of ABCB6. TM helices in ABCB6 amino acid sequence (NP 005680.1) were predicted using A) HMMTOP or B) TMHMM. Topology model representation was generated by TMRPres2D ([248]

http://bioinformatics.biol.uoa.gr/TMRPres2D). The additional luminal loop in HMMTOP is boxed. C) Glycosylation site insertion shows that TMHMM prediction appears to be valid. N-glycosylation sites were introduced into N6Q mutant lacking inherent glycosylation site and the mutants were analyzed for glycosylation status using PNGase F. 

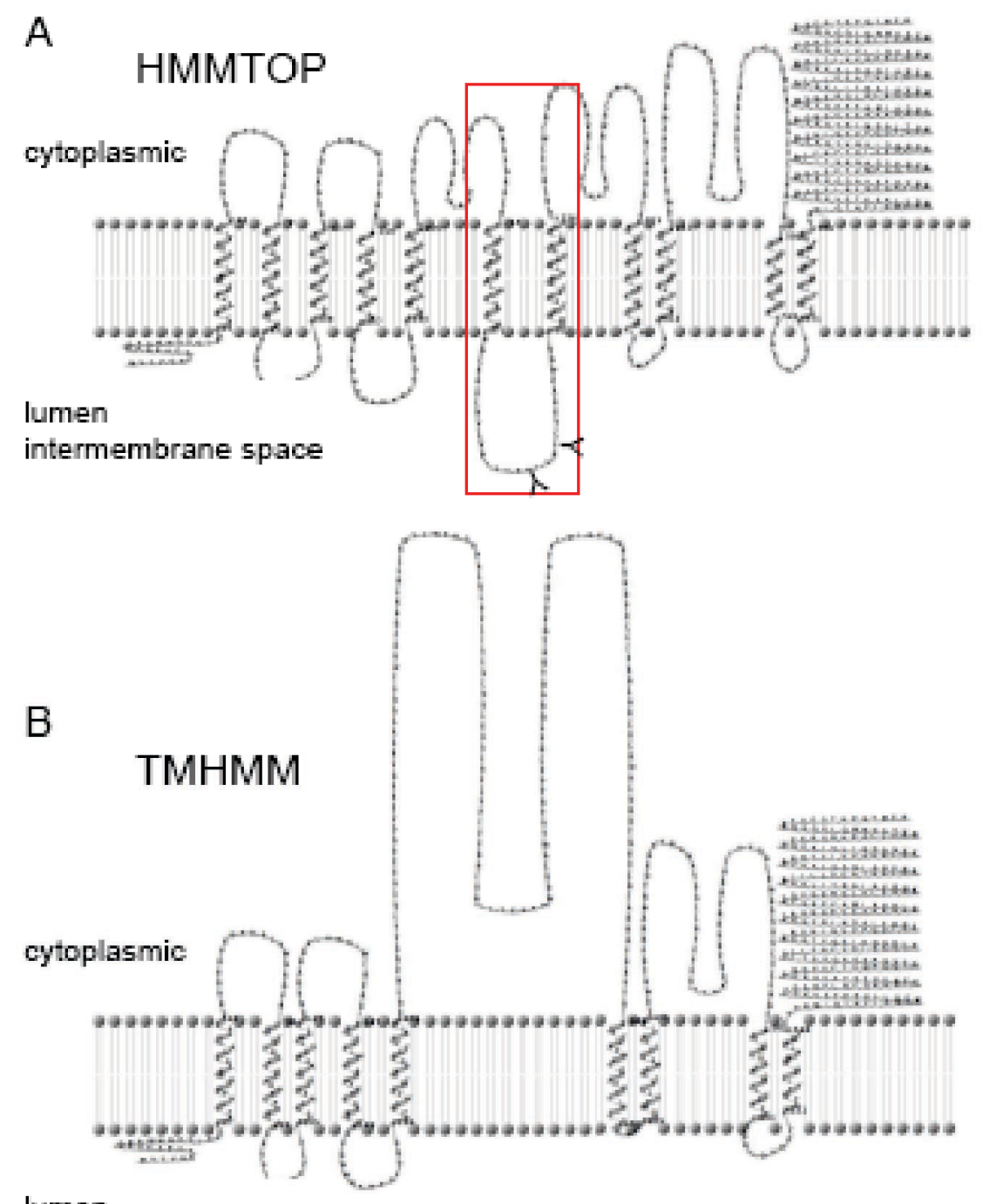

lumen

intermembrane space

$\mathrm{C}$

ABCB6 N6Q Q236NV290T L292N
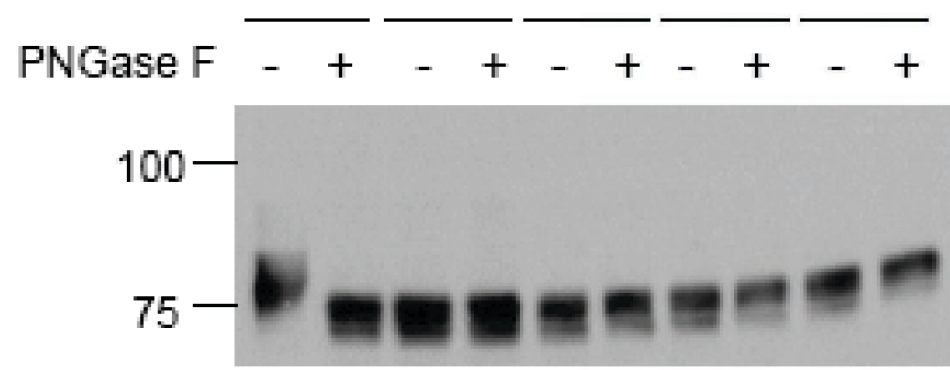

anti-V5 
In order to validate the membrane topology of $\mathrm{ABCB}$, consensus glycosylation sites were introduced into the non-glycosylated form of ABCB6, ABCB6-N6Q at positions V290T and L292N). If HMMTOP prediction is valid, then V290T and L292N mutants, which contain perfect consensus sites for glycosylation at Asn288 or Asn290 in the additional luminal loop predicted only in HMMTOP, will be glycosylated. These ABCB6 constructs along with an unmodified ABCB6 were transiently expressed in NIH3T3 cells and lysates were treated with PNGase F. The PNGase F sensitivity of each mutant was analyzed by immunoblotting (Fig. 4.3.). An additional ABCB6 control was developed in ABCB6-N6Q where Glu236 was substituted with asparagine residue (Q236N) to make a consensus glycosylation site in the predicted cytoplasmic loop. Accordingly, this mutant ABCB6 was not glycosylated. Moreover, V290T and L292N were not glycosylated as only the fast migrating band was observed even in untreated samples. Because these sites were not accessible to glucosyltransferase, they are likely to reside in the cytoplasm, favoring the membrane topology predicted by TMHMM.

\subsubsection{Cysteine Is Dispensable for Glycosylation but Is Critical for ER Exit}

Since ABCB6 is only glycosylated at the atypical glycosylation motif, we next tested if the cysteine in $\mathrm{N}-\mathrm{X}-\mathrm{C}$ was required for glycosylation of ABCB6. The cysteine residue was substituted with a serine residue to make a consensus glycosylation site $(\mathrm{N}$ $\mathrm{X}-\mathrm{S}$, labeled C8S). The C8S mutant was transiently expressed in NIH3T3 cells and glycosylation state was evaluated by PNGase F treatment. The ABCB6-C8S was PNGase $\mathrm{F}$ sensitive indicating that the cysteine residue is not required for glycosylation (Fig. 4.4.). As a control, we showed that glycine substitution to form a non-consensus site caused a loss of glycosylation, which underscores the importance of having either the consensus N-X-S/T or N-X-C motif for glycosylation. Unexpectedly, C8S mutant was Endo $\mathrm{H}$ sensitive indicating that this mutant failed to exit the ER (Fig. 4.4.).

Despite glycosylation, the retention of ABCB6-C8S in the ER suggested this residue might play a role in ER to Golgi trafficking. We found that Cys8 is highly conserved among human, chimpanzee, mouse, rat, and zebrafish (Fig.4.5.A ) suggesting a conserved function of this residue across species. Notably, the asparagine residue for glycosylation in not conserved in zebrafish. This analysis also revealed a conserved downstream cysteine residue at position 26. Based on our computer-assisted analysis, both TMHMM and HMMTOP predict Cys26 would be in the ER lumen. To determine if Cys26 played a role in ER to Golgi trafficking, we mutated the cysteine residue to alanine or serine residue in the wild-type and C8S mutant. Single mutants, C26A and C26S, were both PNGase F and Endo H sensitive like the trafficking-impaired ABCB6-C8S (Fig. 4.5.B). Furthermore, the mutant lacking both cysteine residues, $\mathrm{C} 8 \mathrm{~S} / \mathrm{C} 26 \mathrm{~A}$ and $\mathrm{C} 8 \mathrm{~S} / 26 \mathrm{~S}$, were Endo $\mathrm{H}$ sensitive. These results indicate that both Cys 8 and Cys 26 are critical for the ER exit of ABCB6. We hypothesized that we could rescue trafficking defect by inserting a cysteine residue just downstream from $\mathrm{C} 8 \mathrm{~S}$; therefore, a cysteine residue was inserted between Glu9 and Ala10 to create C8S/C10in mutant. However, this construct failed to acquire Endo $\mathrm{H}$ resistance, which indicates that the space between the two cysteines is important to ER exit of ABCB6 (Fig. 4.5.C). 
A

N6Q: QYC

C8S: NYS

C8G: NYG

Q4: N447,498,677,775Q

Q5: N6,447,498,677,775Q

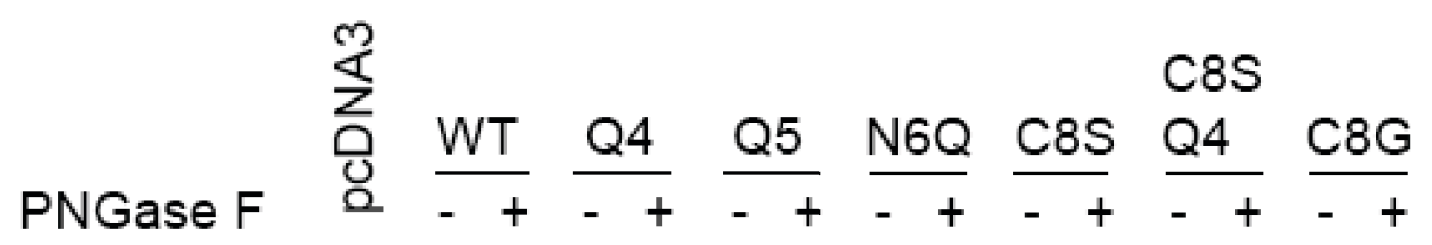

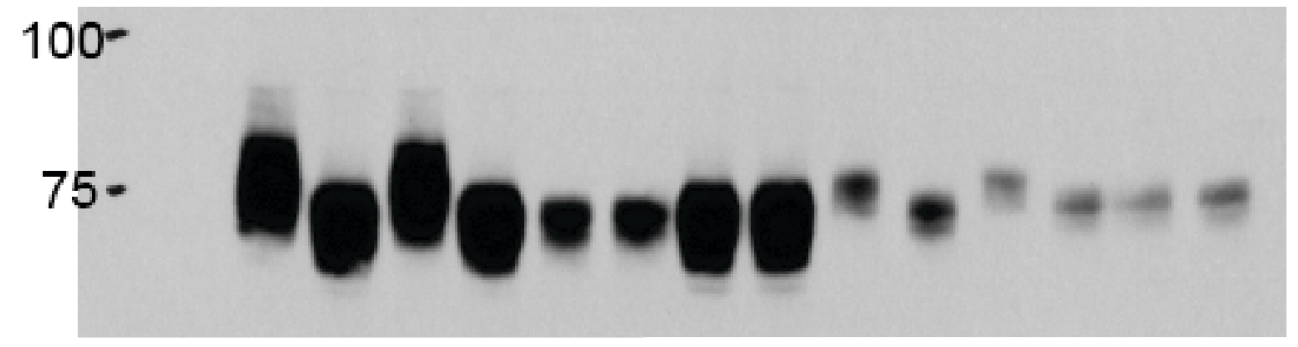

anti- $\vee 5$

$B$

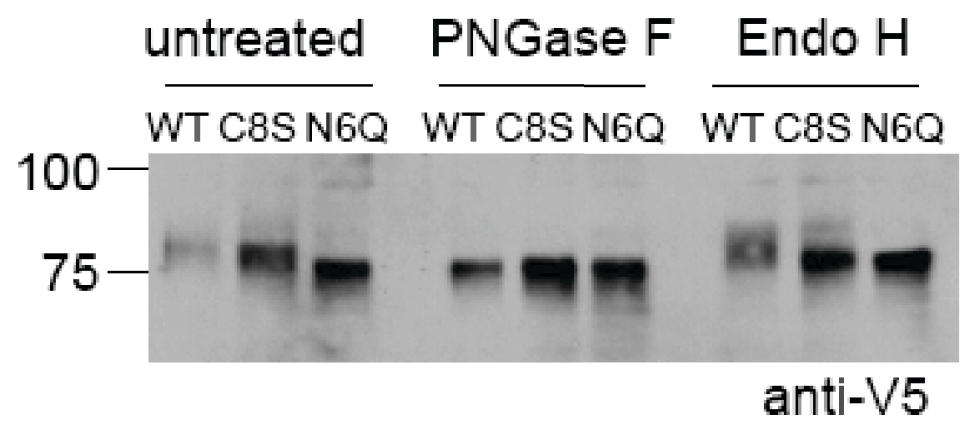

Figure 4.4. Cys8 is dispensable for glycosylation but is critical for ABCB6 ER exit. A) Cys8 is substituted with serine or glycine and the mutant proteins were transiently expressed in NIH3T3 cells. The glycan modification of the mutants was determined by PNGase F sensitivity and analyzed as in Fig. 4.3. ABCB6 is still glycosylated when C8 is substituted with Ser to make a consensus motif. B) Total lysates from NIH3T3 cells expressing either wild type ABCB6, C8S, or N6Q mutants were analyzed for PNGase F and Endo $\mathrm{H}$ sensitivity. 

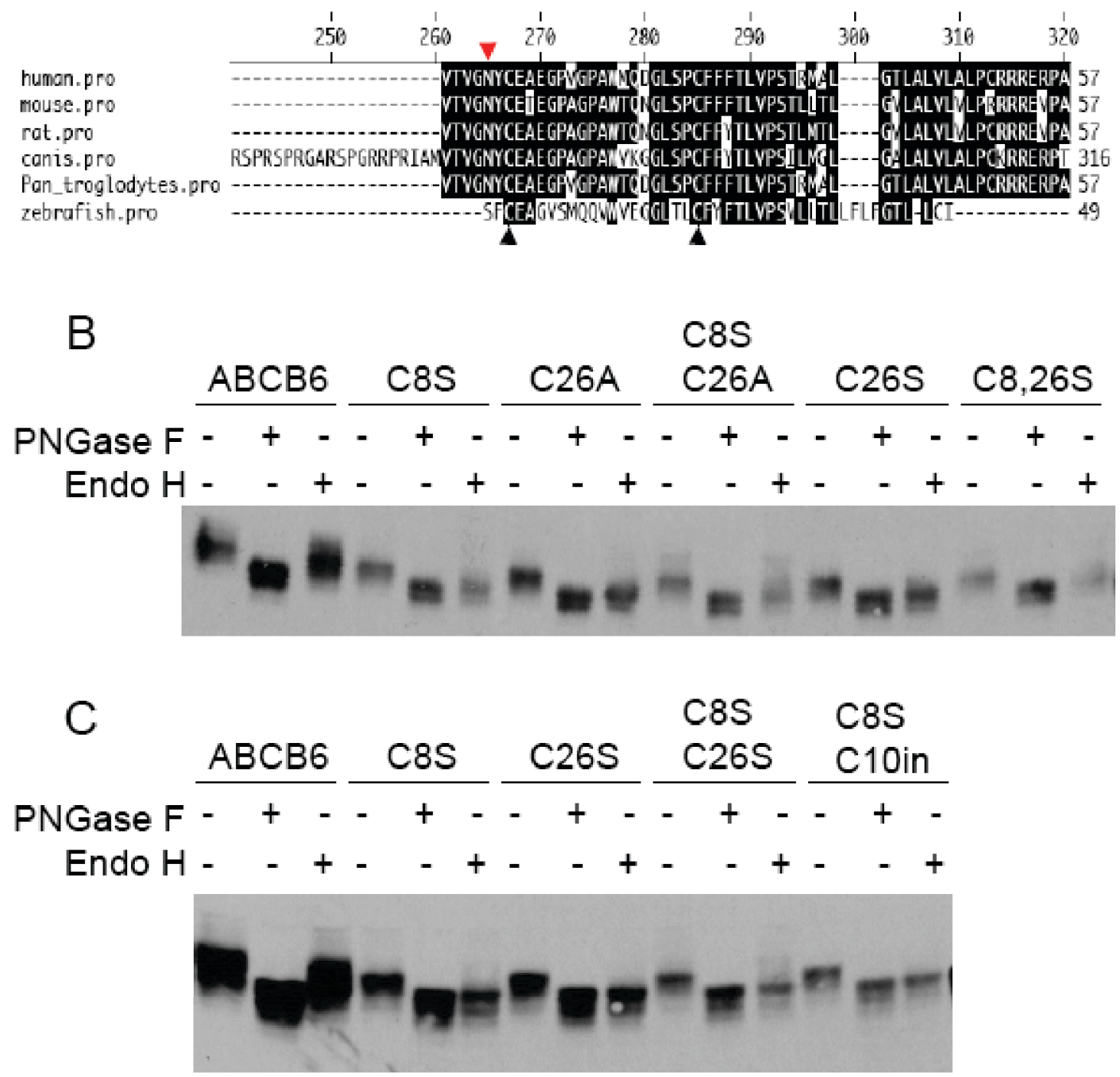

Figure 4.5. Both Cys8 and Cys26 are required for ER exit of ABCB6.

A) Cys8 and Cys26 are highly conserved among species. Amino acid sequence alignment of ABCB6 from human, mouse, rat, dog, chimpanzee, and zebrafish. Only the Nterminus of each sequence is shown. Dog ABCB6 contains a long additional N-termius that is omitted from this figure. Red arrowhead indicates the $\mathrm{N}$-glycosylation site, whereas black arrowheads indicate the conserved cysteine residues. B) Cys26 is substituted with alanine or serine on wild-type or C8S mutant. The mutants were analyzed for glycosidase sensitivity as in Fig. 3. Cys26 mutants and double mutants also failed to exit the ER. C) Cysteine residue was inserted between position 9 and 10 in C8S mutant to create a possible site for disulfide bond formation with Cys26. 


\subsubsection{Cys8 and Cys26 Form Intramolecular Disulfide Bond}

Because two conserved cysteine residues facing the ER lumen are critical for the ER exit of ABCB6, we next tested if they formed an intramolecular disulfide bond. Intramolecular disulfide bonds are detected by mobility shifts of bands in non-reducing compared to reducing SDS-PAGE, because the disulfide bonds produce protein compaction resulting in a faster migration under non-reducing conditions compared to the reduced ones. The extent of the band shift is positively correlated with the distance between the two participating cysteine residues. We reasoned that the minimal number of residues separating the two conserved cysteines in ABCB6 (17 A.A.) would produce a barely detectable band shift in a protein with a molecular mass of $94 \mathrm{kDa}$. Therefore, we developed an ABCB6 chimeric protein that contained 210 amino acids from the Nterminus fused to GFP. The mutant exhibited PNGase F sensitivity and Endo $\mathrm{H}$ resistance, the pattern similar to full length ABCB6 (Fig. 4.6.B). According to the membrane topology prediction, N1-210 mutant spans the membrane five times and places the tagged-C-terminus in the cytoplasm (Fig. 4.6.A). To distinguish the band shift better, the same truncation mutant was tagged with a Flag epitope instead of a bulky GFP molecule. This construct has a further advantage because the different forms of nonglycan and glycan modified ABCB6 can be identified. In order to eliminate any contaminating effects from the other cysteine residues in the construct (inter- or intramolecular disulfide bonds via other cysteines), Cys50 and Cys 120 were substituted with alanine residues in the mutant construct $\left(\mathrm{ABCB} 6_{\mathrm{N} 1-210}\right.$-Flag and $\mathrm{ABCB} 6-$ C50/120A $\mathrm{A}_{\mathrm{N} 1-210}$-Flag).

Three distinct bands were readily seen upon analyzing ABCB6C50/120 $\mathrm{A}_{\mathrm{N} 1-210^{-}}$ Flag by immunoblotting (Fig. 4.6.C). Glycosidase assays allowed us to determine the identity of each band: the slowest migrating band (a) is PNGase F sensitive and Endo H resistant band and represents the mature ABCB6 with complex glycans, the middle band (b) is PNGase F and Endo H sensitive and represents ER-type ABCB6 with high mannose modification, and the fastest migrating band (c) contains no glycans and is, therefore, the immature $\mathrm{ABCB} 6$. The size of the truncated $\mathrm{ABCB} 6$ allows separation of the very distinct forms of $\mathrm{ABCB} 6$ unlike the full-length $\mathrm{ABCB} 6$.

We next transfected ABCB6 $6_{\mathrm{N} 1-210}$-Flag and ABCB6-C50/120A $\mathrm{A}_{\mathrm{N} 1-210}$-Flag into $\mathrm{K} 562$ cells and analyzed the proteins in the presence or absence of reducing agent DTT. Cells were treated with and harvested in the presence of alkylating agent NEM to prevent spontaneous disulfide bond formation. ABCB6 samples ran in the absence of DTT migrated faster compared to the reduced samples suggesting that $\mathrm{ABCB} 6$ has different folding under non-reducing conditions due to a disulfide bond (Fig. 4.6.D).

\subsubsection{N-terminal Cysteine Residues Are Conserved in the Long MRPs}

The "core" unit of $\mathrm{ABC}$ transporters consists of a membrane-spanning domain (MSD) with six TM helices and a nucleotide-binding domain (NBD) [87]. Functional transporters require two sets of such core units; full transporters contain two units in 
A

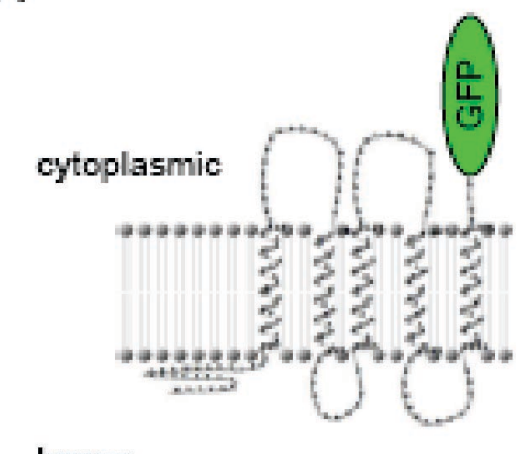

Iumen

intermembrane space
$\mathrm{B}$

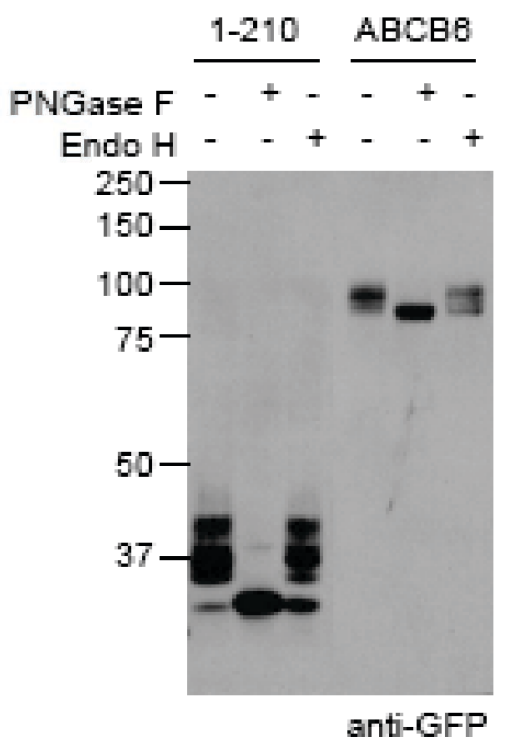

$\mathrm{C}$

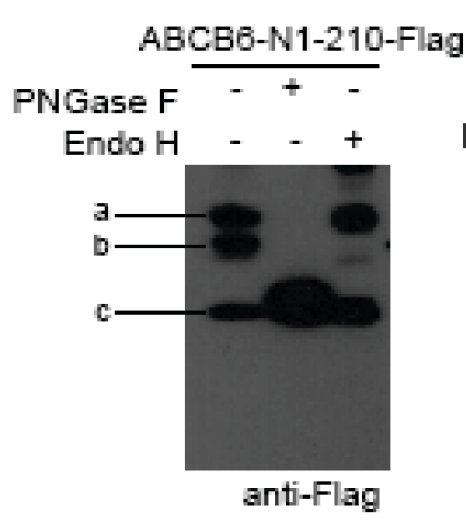

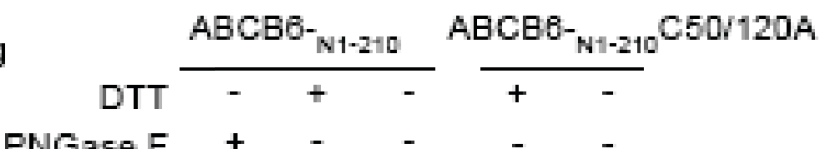

PNGase F

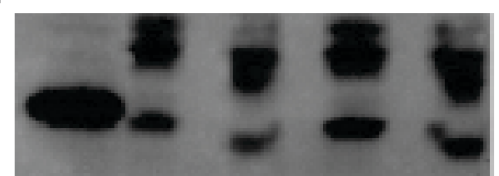

anti-FIAg

Figure 4.6. N-terminus of $\mathrm{ABCB} 6$ contains a disulfide bond.

A) Schematic drawing showing that $\mathrm{ABCB} 6_{\mathrm{N} 1-210}$ mutant spans the membrane five times based on TMHMM prediction with GFP in the cytoplasm. B) ABCB6 $6_{\mathrm{N} 1-210}$ truncated mutant is processed similarly to the full-length protein. Typical results from glycosidase assays are shown. Mouse ABCB6 construct with a C-terminal GFP were used for these experiments. N1-210 showed very low Endo H sensitivity. C) ABCB6-C50/120A $\mathrm{A}_{\mathrm{N} 1-210^{-}}$ Flag was analyzed as in Fig. 4.3. Identity of each band is described in the text. D) $\mathrm{ABCB}_{\mathrm{N} 1-210}$-Flag and ABCB6-C50/120A $\mathrm{A}_{\mathrm{N} 1-210}$-Flag were expressed in K562 cells. K562 cells were treated with NEM for 10 min and then the lysates were prepared in the presence of NEM. Samples were separated on $12.5 \%$ SDS-PAGE with or without reducing agent DTT and analyzed by immunoblotting. 
tandem in a single polypeptide and half transporters with one such unit either need to homo- or hetero-dimerize. Since most ABC transporters contain an even number of TM helices ( of ABCC subfamily, however, contain additional MSD with $5 \mathrm{TM}$ helices N-terminal to the MSD1 placing the N-terminus at the luminal or extracellular side [249]. Therefore, we investigated whether the N-terminal cysteine residues found in ABCB6 were conserved in some of these long MRPs. The alignment of amino acid sequence from $\mathrm{ABCC} 1, \mathrm{C} 2, \mathrm{C} 3, \mathrm{C} 6, \mathrm{C} 8, \mathrm{C} 9$, and $\mathrm{B} 6$ using clustal $\mathrm{V}$ showed that the two cysteines are conserved in all of them with a slight variation in the distance between two residues (17 to 25 amino acids, Fig. 4.7.).

SUR1/ABCC8 is a $\mathrm{K}^{+}$channel regulator involved in modulating insulin secretion at the plasma membrane. In collaboration with Dr. Joseph Bryan, we isolated a mutant SUR1/ABCC8 where the conserved cysteine residue is mutated from a patient with defective $\mathrm{K}^{+}$channel function, and therefore, with aberrant insulin secretion. To test whether the Cys mutant SUR1/ABCC8 was also ER retained as seen in ABCB6, we generated mutant constructs where Cys 8 or Cys 26 is mutated. The remaining 27 cysteine residues have been substituted with alanine or serine residues. The mutant SUR1 proteins were co-expressed with $\mathrm{K}^{+}$channel, $\mathrm{K}_{\mathrm{IR}} 6.2$ in Cosm6 cells and were labeled with [ $\left.{ }^{125} \mathrm{I}\right]-$ azidoglibenclamide. Proteins were fractionated by SDS-PAGE and the mature glycosylated SUR1 was identified by a lower mobility on the gel. The presence of the mature glycosylated SUR1 correlated with its surface expression (data not shown). As reported previously, mature form of SUR1 was observed only when $\mathrm{K}_{\mathrm{IR}} 6.2$ was coexpressed. Notably, the mature form of SUR1 was absent when one or both of the conserved cysteine residues were substituted with alanine or serine residues (Fig. 4.7.).

\subsection{Discussion}

\subsubsection{N-Terminal Disulfide Bond Regulates ER Exit of ABC Transporters}

The mitochondrial membrane localization of the porphyrin transporter ABCB6 has been shown by three independent studies [171, 204, 250]. Additionally, we, and others, have shown that ABCB6 specifically localizes to the mitochondrial outer membrane [204, 250]. Nonetheless, how ABCB6 reaches the mitochondrial outer membrane is important to understanding its function. An important key was our finding that ABCB6 is modified by glycan addition. Moreover, ABCB6 utilizes a single unique glycosylation site that is conserved among mammalian ABCB6, but heretofore only found in secreted proteins. This unique glycosylation site led to the identification of two conserved cysteine residues that are found in many $\mathrm{ABC}$ transporters and mutation within one ABCC family member (SUR1/ABCC8) is the genetic basis for the loss of function due to impaired membrane trafficking.

In this study, we demonstrate that the two cysteine residues in the N-terminus of ABCB6 and SUR1 are an important ER exit signal. Substitution of cysteine residues in 


\section{A}

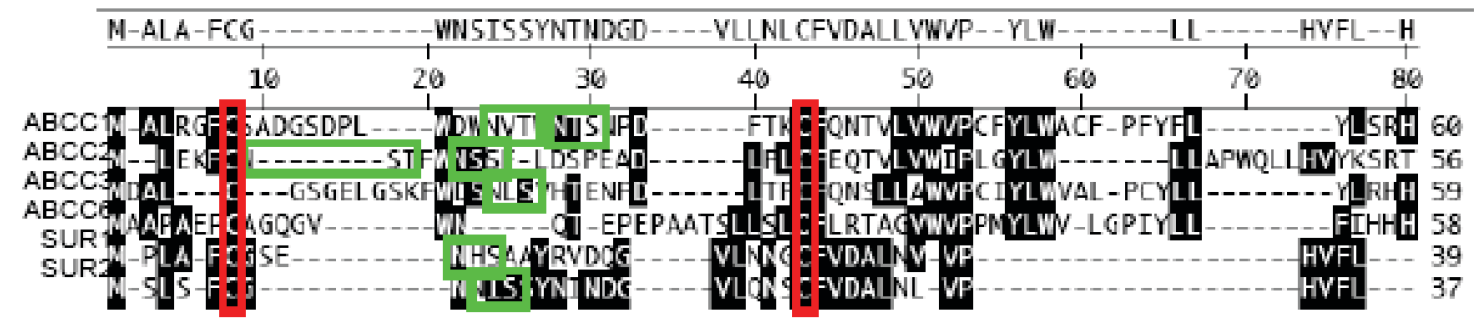

B

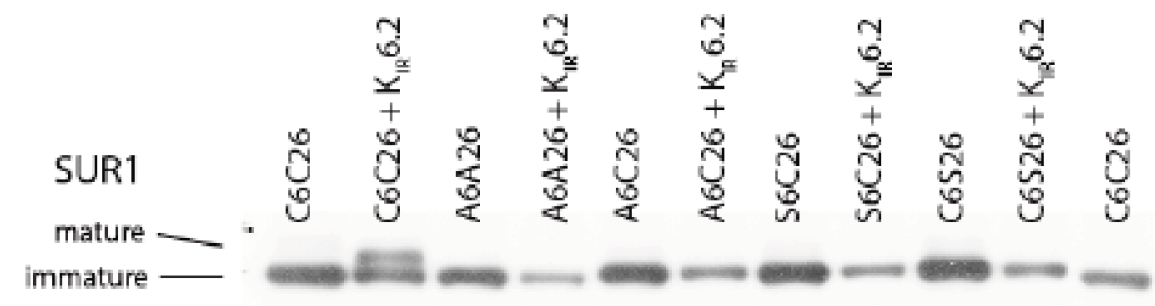

Figure 4.7. N-terminal cysteines are conserved in long MRPs and regulate trafficking.

A) Amino acid sequence alignment of $\mathrm{ABCC} 1, \mathrm{C} 2, \mathrm{C} 3, \mathrm{C} 6, \mathrm{C} 8$, and $\mathrm{C} 9$ is shown. Conserved cysteine residues are boxed in red and glycosylation sites are boxed in green. B) SUR1/ABCC8 constructs containing mutations at Cys6 and/or Cys26 were coexpressed with KIR6.2 in COSm6 cells. SUR1 was labeled with [125I]azidoglibenclamide, fractionated on SDS-PAGE, and detected by autoradiography. 
either ABCB6 or SUR1 with either serine or alanine at conserved positions leads to ER retention. This finding suggested one of the two related possibilities: i) the disulfide bond formation between ABCB6 Cys8 and Cys26 provides a loop that is a signal for ER exit or ii) a reduced cysteine(s) is a signal for ER retention. We favor the former idea because while single cysteine mutants are retained, double mutations of Cys 8 and Cys 26 that have no cysteine also results in a dramatically impaired ER exit. We confirmed that the Nterminus of ABCB6 forms an intramolecular disulfide bond between Cys 8 and Cys 26 by showing that the chimeric $\mathrm{ABCB} 6_{\mathrm{N} 1-210}$-Flag migrated faster under non-reducing conditions. These results indicate that disulfide bond-dependent folding occurs in the Nterminus. Further support for a specific disulfide loop in ABCB6 being required for ER exit is the finding that an insertion of a cysteine residue at position 10 does not rescue trafficking defect of ABCB6-C8S mutant.

The ER exit signal, which ABCB6 employs, appears to be different from that of the thiol retention mechanism, where exposed thiols are recognized by ER protein(s) as a retention signal [234]. First, most of the proteins that are regulated by thiol retention form intermolecular disulfide bonds [234] unlike ABCB6 where an intramolecular bond is formed. Second, in thiol retention, loss of the cysteine residue involved in the disulfide bond formation usually results in an increased secretion presumably by a lack of interaction with ER resident protein(s) that normally results in the ER retention [235, 236]. On the other hand, in ABCB6, lack of a disulfide bond due to mutations of both cysteine residues involved does not rescue the defective ER exit. The disulfide loop may act as an ER exit signal via two possibilities: i) chaperone proteins dissociate upon the "disulfide-bonded loop" formation as a result of loss of a cysteine-independent binding site or ii) the structural change caused by the disulfide-bonded loop may provide a binding site for other chaperone proteins. At this point, these two possibilities have not been verified and are subject for future studies.

Like ABCB6, the N-terminal cysteines required for ER exit are found in ABCC subfamily members that contain an $\mathrm{N}$-terminus that inserts into the ER and is glycosylated (long MRPs; ABCC1, C2, C3, C6, C8, and C9) [249]. It is notable that nonfunctional $\mathrm{ABCC} 8 / \mathrm{SUR} 1$ from a hyperinsulinemic hypoglycemia patient had a single point mutation in in this conserved cysteines. This single mutation disrupted the potassium channel function in a hyperinsulinemic hypoglycemia patient. The mutant SUR1/ABCC8 is ER retained as shown by a lack of mature glycosylated SUR1/ABCC8. Thus, the N-terminal cysteines found in ABCC8/SUR1 also serve as an ER exit signal, most likely by through the formation of a disulfide bond. Further support for the role of the N-terminus is found in studies where the N-terminus of ABCC1 and ABCC2 localize to the plasma membrane in the absence of rest of the protein $[251,252]$ and ABCC1 lacking this region (MSD0) has reduced cell surface expression [252]. These results further support our findings that the $\mathrm{N}$-terminus, which contains the cysteines, is essential for trafficking of ABCC family members that insert their N-terminus in the ER.

However, disulfide bond formation may be important in other ABC transporters [233]. In ABCG2, Cys592 and Cys608 form an intramolecular disulfide bond [253]. It is notable that the spacing between these cysteines is 16 amino acids, which is close to the separation conserved in ABCB6 and the ABCC family members. The ABCG2 double 
cysteine mutant, C592/608A, lacked BODIPY-prazocin efflux activity and was expressed at a very low level on the cell surface. These results lend further support to our findings that the formation of intramolecular disulfide bond is a critical component of ER exit for many $\mathrm{ABC}$ transporters.

\subsubsection{ABCB6 Is Processed in the ER and Golgi}

In present study, we show that the mitochondrial protein, ABCB6 contains glycan modifications, which reveal trafficking from the ER to the Golgi. The presence of glycoproteins in mitochondria has been previously shown although the trafficking of such glycoproteins to mitochondria from the ER is not fully understood [254]. Translocation from the mitochondria to the ER was suggested when hepatitis $C$ virus core protein showed either ER or mitochondrial localization [255]. The core protein localized to the ER when highly expressed by the CMV promoter but showed mitochondrial localization when expressed at much lower levels from a tetracycline-regulated promoter [255]. Interestingly, both the hepatitis $\mathrm{C}$ virus core protein and human cytomegarovirus UL37 glycoprotein, another viral protein, localize to the ER prior to the mitochondrial translocation [256]. However, these studies do not provide insight into how ABCB6 moves from the Golgi to the mitochondria.

The N-glycosylation site in ABCB6 is unique because a cysteine residue (N-X-C) is substituted instead of a serine or threonine as found in most glycoproteins [240]. The atypical N-X-C motif has been reported only in five other proteins [241-245]. However, the functional role of this atypical motif is unknown because these proteins contain typical glycosylation sites, which might mask the function of the atypical site. We show that the cysteine residue in $\mathrm{ABCB} 6$ is not required for glycosylatin because substitution with a serine residue in the N-glycosylation motif does not affect ABCB6 glycosylation. Nontheless, analysis of ABCB6 from different species reveals that the asparagine residue is not conserved in zebrafish whereas the cysteine residue is. This suggests that genomic sequence upstream of the codon encoding the conserved cysteine residue acquired an $\mathrm{N}$ glycosylation site in mammals.

Our study suggests ABCB6 topology is different from other half transporters. We have previously shown that the C-terminus of ABCB6 is localized to the cytoplasm [204]. The ER luminal localization of the N-terminus of ABCB6 indicates a protein with an odd number of TM helices which places the $\mathrm{N}$ and $\mathrm{C}$-termini on the opposite sides of the ER membrane. This finding eliminated the proposed ABCB6 topological model with an even number of TM domains [250]; however, it could not distinguish if ABCB6 had nine TM helices (predicted by TMHMM) or 11 TMs (predicted by HMMTOP). To reconcile these two models, we inserted artificial glycosylation sites. The TMHMM membrane topology reveals the presence of a large cytoplasmic loop 3 (amino acids 206382). Interestingly, the naturally occurring ABCB6 splice variant, termed PRP, lacks 50 amino acid residues within this loop. Since PRP does not bind to hemin-agarose as assessed by pull-down assays [204], we speculate that this loop may be a heme-binding domain. 


\subsubsection{Physiological Implication of the N-Terminal Disulfide Bond}

The $\mathrm{ABC}$ transporters play important biological roles and provide the means to regulate the concentration of toxic xenobiotics and metabolites. To fine tune their arrival at their destination, both cis and trans mechanisms must be available to the cell. Our studies show that among many $\mathrm{ABC}$ transporters, formation of a disulfide bond between two conserved cysteines is essential for ER exit. Intramolecular disulfide bonds have been implicated in the post-trans Golgi network sorting of the secretory proteins, although the effect of DTT on the sorting of proteins was protein and cell type dependent [257]. For choromograin B, a secretory protein found in many endocrine cells and neurons, an N-terminal disulfide bond (Cys16 and Cys37) has been shown to play an important role in sorting into regulated secretory granules rather than constitutive secretory granules [257]. Although the disulfide bond we identified in this study determines the ER to Golgi movement, unlike chromograin B, the use of disulfide bonds as a sorting signal underscores the importance of proper post-translational modifications for protein trafficking.

Interestingly, our data suggest that in a condition where the disulfide-bonded loop does not form, $\mathrm{ABCB} 6$ will be ER retained and imply that this occurs for other $\mathrm{ABC}$ transporters. In the oxidized environment of ER lumen, the ratio of oxidized to reduced glutathione (GSSG and GSH, respectively) is much higher compared to that of the cytoplasm and the glutathione acts as buffer to maintain the redox state. Several pathophysiological conditions such as aging are known to accompany changes in the cellular redox state, mostly to the oxidative state. While oxidative stress has been reported in diabetes, a reductive shift was observed in the microsomal redox status in rats with streptozotocin-induced diabetes [258]. Our results suggest that the ditribution patterns of functional $\mathrm{ABC}$ transporters may be altered in conditions where the ER redox status is shifted.

The $\mathrm{K}^{+}$channel $\left(\mathrm{K}_{\mathrm{IR}}\right)$ and its regulator, SUR1/ABCC8, require oligomerization to properly target the cell surface [259]. The newly identified conserved cysteines and presumably the disulfide-bonded loop in SUR1 adds another layer of checkpoint to ensure that only properly modified proteins leave the ER. While it is difficult to identify an universal signal for the sorting and trafficking of all $\mathrm{ABC}$ transporters, a small disulfide-bonded loop appears necessary for ER egress of a group of $\mathrm{ABC}$ transporters. 


\section{CHAPTER 5. DISCUSSION}

The $\mathrm{ABC}$ transporters regulate the transmembrane movement of various compounds in an ATP-dependent manner [2]. At the plasma membrane, ABC transporters provide an important barrier to protect cells and tissues from the accumulation of toxic compounds; however, their ability to exclude different classes of drugs is the basis for many MDR phenotypes in tumor cells [53]. The physiological roles of $\mathrm{ABC}$ transporters extend beyond the barrier function and their importance in many biological processes is highlighted by their link to several pathophysiological conditions. For example, mutations in the $\mathrm{ABC}$ genes are the genetic basis for many human disorders including cystic fibrosis, Dubin-Johnson syndrome, immunedeficiency, and X-linked sideroblastic anemia and ataxia [2]. Although deficiencies of functional ABC transporters in the humans and the animal models revealed diverse physiological roles of $\mathrm{ABC}$ transporters, there are still many $\mathrm{ABC}$ proteins with unknown physiological functions. $\mathrm{A}$ relatively uncharacterized $\mathrm{ABC}$ transporter, $\mathrm{ABCB} 6$, is a mitochondrial half transporter with a possible role in iron homeostasis [171]. These previous findings, coupled with our findings that ABCB6 is highly expressed in the site of definitive erythropoiesis and its expression is coordinated with heme biosynthesis, prompted us to determine its role in heme biosynthesis. Our results that ABCB6 binds to various porphyrins, shows CPIIIstimulated ATPase activity, and mediates ATP-dependent heme uptake into mitochondria all support our hypothesis that ABCB6 is a porphyrin transporter [204]. Moreover, ABCB6 is ideally located on the mitochondrial outer membrane to transport an anionic heme precursor, CPgenIII, into the intermembrane space where the next enzyme in the pathway resides. Indeed, overexpression of ABCB6 accelerates de novo porphyrin biosynthesis suggesting that ABCB6 facilitates the process by overcoming the energetically unfavorable transmembrane movement of porphyrins. By using a heminagarose pull-down assay we developed, we demonstrated that ATP binding to ABCB6 is sufficient to induce a conformational change, which lowered its affinity for its substrate. Our results are in agreement with a recently published structural data of nucleotide-bound bacterial $\mathrm{ABC}$ protein. Three other mitochondrial $\mathrm{ABC}$ transporters have been shown to reside in the inner membrane, and thus, ABCB6 is the only one to localize on the outer membrane reported so far. The mitochondrial outer membrane localization has later been shown by a different group confirming our results [250]. However, recent studies report ABCB6 localization on different cellular membranes including the plasma membrane, Golgi, and vesicles [250, 260, 261]. Therefore, how ABCB6 trafficks intracellularly has been poorly understood. Our results here demonstrate that ABCB6 contains Endo $\mathrm{H}$ resistant glycans, which indicates ER to Golgi movement prior to the mitochondrial localization. The characterization of the glycan modification on the atypical N-X-C motif uncovered conserved cysteine residues that are important for ER exit of ABCB6. These cysteines are found in several other $\mathrm{ABC}$ family members and mutation in the conserved cysteine residue in ABCC8 results in the lack of its cell surface expression and is the genetic basis for hyperinsulinemic hypoglycemia in a patient. 


\subsection{ABCB6 Is a Homodimeric Mitochondrial Porphyrin Transporter}

Substrates of ABC transporters are generally tested for one or more of the following characteristics: the binding to the transporters, the ability to stimulate ATPase activity, and the ATP-dependent uptake by the transporters. Substrate binding and the uptake assays are typically limited by an availability of radiolabeled compounds. In order to circumvent the use of radiolabeled hemin, our lab has previously utilized a pull-down assay using hemin conjugated to agarose to test the interaction between ABCG2 and heme [75]. ABCB6 binds to hemin-agarose specifically and the interaction can be disrupted by an addition of various porphyrins. Interestingly, only the compounds with tetrapyrrole ring were able to displace ABCB6 from hemin-agarose effectively. CPIII, an oxidized form of heme precursor, CPgenIII, was the most effective porphyrin tested in displacing ABCB6 from hemin-agarose. The commercially available CPIII is used throughout this study instead of the physiologically relevant CPgenIII, because CPgenIII is readily oxidized to CPIII in the atmosphere. Another heme precursor, PPIX and a plant porphyrin, $\mathrm{PhA}$, also competed for binding of ABCB6 to hemin-agarose to a lesser extent. A monopyrrole, PBG, did not affect the interaction between ABCB6 and heminagarose even though it is a heme precursor suggesting that ABCB6 recognizes a specific structure. Moreover, vitamin $\mathrm{B}_{12}$, which has a tetrapyrrole backbone as well as a bulky side chain, was not effective in displacing ABCB6 from hemin-agarose. These results show that $\mathrm{ABCB} 6$ recognizes anionic compounds with a tetrapyrrole ring, but the binding pocket cannot accommodate a bulky side chain.

The substrate binding promotes ATP binding and a formation of NBD sandwich dimer, which results in stimulation of ATP hydrolysis. In order to measure ATPase activity of mitochondrial ABCB6, a cell line stably expressing non-functional Walker A mutant ABCB6 was established. The Walker A mutant ABCB6 contained glycine residue in place of conserved lysine residue the Walker A domain, a mutation that has been shown to disrupt ATP binding or hydrolysis in other ABC transporters [212, 213, 223]. An increase in the vanadate sensitive ATPase activity was observed in only the mitochondria prepared from K562 cells expressing wild type ABCB6-V5 but not in the Walker A mutant-V5. The modest increase may be attributed to the following factors: i) incomplete inhibition of $\mathrm{F}_{1} \mathrm{Fo}$-ATPase in the mitochondria masked the much smaller ABCB6 ATPase activity and ii) both ATPase and transport acitivity of ABCB6 bearing a V5 tag was lower compared to the Flag-tagged ABCB6 for unknown reasons. Because the Walker A mutant cell line was established using the V5-tagged molecule, only the results from the V5-tagged ABCB6 are presented here. The Walker A mutant binds hemin as assessed by hemin-agarose pull-down assay, which was a useful feature when testing the uptake of hemin into mitochondria. Lack of ATP-dependent uptake of radiolabeled hemin into the Walker A mutant expressing mitochondria demonstrated that the assay measures the actual transport rather than hemin simply binding to the ABCB6 at the membrane. Taken together, hemin meets all the criteria to be a substrate for ABCB6 and our results imply that other porphyrin are likely to be substrates as well.

ABCB6 is a half transporter with one MSD and one NBD; therefore, it must homo- or heterodimerize in order to reconstitute transport function. We hypothesized that 
ABCB6 is likely to form a homodimer because there is no potential dimerizing partner on the mitochondrial outer membrane. Co-imunoprecipitation experiments using differentlytagged $\mathrm{ABCB} 6$ show that it homodimerizes. The findings that the overexpression of ABCB6 alone was sufficient to observe transport activity, etc., also support the homodimerization of $\mathrm{ABCB}$. Recently, $\mathrm{ABC}$ transporters have been shown to contain multiple substrate binding sites/pockets that can accommodate structurally different compounds possibly at the same time [107, 115, 226]. A homodimeric half transporter ABCG2 has been proposed to contain multiple substrate binding pockets [54]. By creating a dimerization-deficient $\mathrm{ABCB} 6$, we wanted to test whether porphyrin binding was retained in a single molecule. This would provide some insights into how substrate binding sites are constituted in ABCB6. However, despite the generation of multiple mutants, we were unable to identify domain(s) that are important for dimerization, which may be an indication that the dimer is stabilized through multiple interaction sites.

\subsection{ABCB6 Accelerates Porphyrin Biosynthesis}

Overexpression of ABCB6 increases a steady state PPIX levels in K562 cells. This effect was dependent on function of $\mathrm{ABCB} 6$ because $\mathrm{K} 562$ cells with the Walker A mutant ABCB6 showed no change in cellular PPIX levels compared to vector cells. We further demonstrated that de novo porphyrin biosynthesis was upregulated in cells expressing functional ABCB6. Heme biosynthesis takes place in two compartments requiring transmembrane movement of intermediates: ALA into the cytosol from mitochondria and CPgenIII into the mitochondrial intermembrane from the cytosol. The upregulation of porphyrin biosynthesis by $\mathrm{ABCB} 6$ and its function as the mitochondrial outer membrane transporter pumping porphyrins into the intermembrane space from the cystosol suggest that ABCB6 plays an important role in heme biosynthesis. Based on these results, we propose that ABCB6 is the elusive CPgenIII transporter in heme biosynthesis pathway, which had been an unanswered question in the field for a long time. CPgenIII readily associates with the membrane via its hydrophobic cyclic rings; however, the anionic side chains do not allow the transmembrane movement to occur easily. Indeed, previous studies show that the rate of simple diffusion of CPgenIII is not sufficient to support robust heme biosynthesis in differentiating erythroid cells and the presence of energy-dependent transport has been suggested [192, 193]. The movement of CPgenIII may represent yet another rate-limiting step in heme biosynthesis that ABCB6 can overcome.

\section{3. $\quad$ ATP Binding Releases the Substrate from ABCB6}

The transmembrane movement of compounds by ABC transporters is ATPdependent. Initial biochemical studies led to a model that ATP hydrolysis provided the power stroke for the transport [113]. However, structural data of isolated NBDs and recent biochemical studies suggest that formation of a NBD dimer induced by substrate and/or ATP binding is the power stroke for the substrate transport [97, 114]. This change in the conformation of NBD is then transmitted to MSD, which causes a sufficient 
conformational change to release substrates on the other side of the membrane. Our results support the latter model, referred to as "ATP switch model," in which binding of ATP induces a conformational change to a low affinity state for the substrate of ABCB6. Taking advantage of the affinity of ABCB6 for hemin-agarose, we demonstrated that addition of ATP at room temperature was sufficient to release ABCB6 from heminagarose. Furthermore, non-hydrolyzable ATP anolog, AMPPNP, had a similar effect on the release of $\mathrm{ABCB} 6$ from hemin-agarose, although a higher concentration was required. Effects of mutating the conserved lysine residue in the Walker A domain varies among the $\mathrm{ABC}$ transporters. Walker A mutation in some ABC transporters results in a loss of ATP binding and subsequently ATP hydrolysis, whereas only the ATP hydrolysis is disrupted in others [213]. For ABCB6, loss of the conserved lysine residue resulted in a severely impaired ATP binding. Therefore, as expected, the Walker A mutant ABCB6 remained bound to hemin-agarose after ATP or AMPPNP addition.

Although structural data for mammalian $\mathrm{ABC}$ transporters are not available at this time, the structure of bacterial ABC transporter Sav1866 provided insights into the possible transport mechanism that is likely to be similar in the mammalian counterpart. The cytoplasmic loop from one subunit reaches over to contact the NBD from the other subunit and this interface may be important in transmitting information from NBDs to MSDs and vice versa [97]. The MSDs of nucleotide bound Sav1866 is forming outward wings, which suggests that it is in a position to release its substrate [97]. It has been shown that ATP hydrolysis and subsequent release of Pi and ADP disengages the NBD dimer suggesting that this process is involved in resetting the transporter to a high affinity state for the next transport cycle [114]. Our results show that ATP binding induces a sufficient conformational change and supports the transport mode described as the ATP switch model. However, understanding the precise mechanism requires further investigation. For some full transporters and heterodimeric half transporters, two NBDs are shown to be asymmetrical. The binding and hydrolysis of ATP require two NBDs because a functional ATPase site comprises of domains from the both NBDs [110]. For TAP1/TAP2 heterodimer, only one ATPase site contains consensus Walker B, histidine switch, and ABC signature motifs [111]. The consensus ATPase site is considered to provide the power stroke for peptide translocation by TAP1/TAP2. The combination of one consensus and one degenerate ATPase sites is seen in more than 20 ABC transporters [111], and therefore, the asymmetrical NBDs may reveal an important aspect of substrate transport. Whether a homodimer such as ABCB6 contains an asymmetry in its NBD dimer remains to be seen.

\subsection{ABCB6 Is Glycosylated and Contains a Disulfide-Bonded Loop That Acts as a Trafficking Signal}

The initial identification of ABCB6 showed that it localized on the mitochondria [171]. Subsequently, we, and others showed its localization on the mitochondrial outer membrane [204, 250]. However, recent studies showed the localization of ABCB6 on other subcellular membranes such as the plasma membrane and the Golgi $[250,261]$. Therefore, understanding ABCB6 intracellular trafficking may provide insights into how 
$\mathrm{ABCB} 6$ reaches the mitochondrial outer membrane and other membranes. The objective was to study how ABCB6 trafficks in cells, as well as to identify if any signals for sorting or trafficking exist within ABCB6. The unexpected observation that ABCB6 is modified with Endo $\mathrm{H}$ resistant glycans, mitochondrial proteins are usually not glycosylated, indicated that $\mathrm{ABCB} 6$ trafficks to mitochondria via an unconventional way. Furthermore, the glycosylation site of ABCB6 consists of N-X-C instead of a typical N-X-S/T site. By characterizing the atypical glycosylation site, we demonstrate that the conserved cysteine residue, along with another cysteine residue in the N-terminus, plays an important role in ER exit of ABCB6. Interestingly, these cysteines are conserved in ABCC subfamily members that extrude the N-terminus into the ER lumen during maturation (long MRPs). This motif was functional in at least one of the long MRPs. Loss of the conserved cysteine in SUR1/ABCC8 results in a lack of cell surface expression and the lack of functional SUR1/ABCC8 is the genetic basis for a case of hyperinsulinemic hypoglycemia.

\subsubsection{Formation of Disulfide Bond in ABCB6 Acts as an ER Exit Signal}

Post-translational modifications such as signal sequence cleavage, $\mathrm{N}$ glycosylation, and disulfide bond formation occur and are monitored in the ER to ensure that only properly modified proteins leave the ER. We found that when the cysteine residue (Cys8) in the glycosylation site of ABCB6 is substituted with serine, ABCB6 is still modified but with Endo H sensitive glycans. Glycans are Endo H sensitive when they are attached to proteins in the ER but acquire Endo $\mathrm{H}$ resistance after processing in Golgi. Therefore, Endo H sensitive glycans on C8S mutant indicated that it failed to exit the ER. The only other cysteine residue predicted to be exposed to the ER lumen is Cys26 and it is highly conserved among other species. The mutant ABCB6 where Cys26 is substituted with serine or alanine also contained Endo $\mathrm{H}$ sensitive glycans. We also confirmed that these two conserved cysteine residues, Cys8 and Cys26, form an intramolecular disulfide bond that appears to be glycan-independent. Oxidative state of cysteine residues has been shown to regulate secretion of several proteins including IgM through a mechanism called "thiol retention" [234]. The cysteine residues monitored in thiol retention needs to oxidize through disulfide bond or by other means to exit the ER. ABCB6 does not appear to be regulated by thiol retention because mutation of both Cys8 and Cys26 did not rescue the defective ER exit. Taken together, we propose that the disulfide-bonded loop at the N-terminus is a novel ER exit signal.

How the disulfide-bonded loop acts as an ER exit signal is yet to be determined. However, there are two possibilities: i) chaperones dissociate upon the formation of the loop as a result of loss of a binding site or ii) the loop formation creates a binding site for other chaperone proteins. It will be interesting to determine if there is a stable interaction between $\mathrm{C} 8 \mathrm{~S}$ or C26A mutant and ER resident proteins.

Many ABC transporters contain even number of TM helices and therefore have both $\mathrm{N}$ - and $\mathrm{C}$-termini in the cytosol. However, there is a subset of $\mathrm{ABC}$ transporters in ABCC subfamily, which contain an additional five TM helices N-terminal to the 
MSD1.The glycan modifications on the N-termini of these "long MRPs" indicate that their N-termini extrude into the ER lumen. The conserved cysteines identified in ABCB6 are conserved in the long MRPs, $\mathrm{ABCC} 1, \mathrm{C} 2, \mathrm{C} 3, \mathrm{C} 6, \mathrm{C} 8$, and $\mathrm{C} 9$. The functionality of these cysteines are conserved at least in one of them, ABCC8/SUR1. Loss of one or both of the conserved cysteine residues results in the defective trafficking of SUR1/ABCC8. The mutation in the conserved cysteine residue in SUR1/ABCC8 was isolated from a patient with hyperinsulinemic hypoglycemia indicating that the failure to exit the ER is the cause of the disease. We have also observed some functional consequences to the loss of the conserved cysteine residues in ABCB6. Our preliminary results show that the unlike wild type ABCB6 expressing K562 cells, cells expressing C8S or C26A mutants have steady state PPIX levels that are comparable to the Walker A mutant cells.

\subsubsection{Intracellular Movement of ABCB6}

The glycan modification on ABCB6 was unexpected because normally, mitochondrial proteins reach their destination after being fully translated in the cytosol. There is evidence for proteins moving from the ER to mitochondria. However, how proteins traffick from Golgi to mitochondria is poorly understood. Further investigation is required to identify how $\mathrm{ABCB} 6$ properly targets mitochondria.

The glycosylation at the very N-terminus revealed a unique topology of ABCB6. Half transporters typically contain six TM helices, thus positioning both $\mathrm{N}$ - and C-termini in the cytosol. Because the $\mathrm{N}$-terminus of ABCB6 is in the ER lumen but its C-terminus is in the cytosol, ABCB6 is predicted to contain an odd number of TM helices. Also, the regions that contain consensus $\mathrm{N}-\mathrm{X}-\mathrm{S} / \mathrm{T}$ sites are likely to reside in the cytosol due to the lack of glycosylation. Based on these criteria and the results from the glycosylation site insertion experiment, we favor the membrane topology model predicted by TMHMM, which has nine TM helices. Half transporters in ABCB subfamily, TAP1 and TAP2, have been shown to contain 10 and nine TM helices, respectively. These $\mathrm{N}$-terminal extensions are important for interaction with tapasin. Perhaps, the additional three TM helices in ABCB6 play an important role in regulating its function or act as a binding site for other proteins.

\subsection{Conclusion}

We have characterized the substrate, physiological role, transport mechanism, and trafficking signal of ABCB6. Since many ABC transporters are able to recognize structurally different compounds, many more substrates for ABCB6 may be identified in the future. It will be exciting to see if ABCB6 is involved in other biological processes. Moreover, tumor cells exhibit a high rate of heme biosynthesis, which is the basis for photodynamic therapy [190]. Therefore, whether ABCB6 is upregulated in these cells to facilitate heme biosynthesis will be of interest. The identification of the N-terminal disulfide-bonded loop formed by conserved cysteines has a broad implication for other $\mathrm{ABC}$ transporters as the motif is found in $\mathrm{ABCC}$ subfamily members. $\mathrm{ABCC} 1$ is one of 
the three major drug transporters, and ABCC2, ABCC6, SUR1/ABCC8 and SUR2/ABCC9 are linked to human disorders (Dubin Johnson syndrome, PXE, FPHHI, and DCVT, respectively). Therefore, mutations in the conserved cysteine residues may result in a lack of cell surface expression of these transporters and, therefore, may lead to the above mentioned disorders. Furthermore, our results suggest that the cell surface expression pattern of these transporters may be altered in pathophysiological conditions that affect ER redox status. 


\section{LIST OF REFERENCES}

1. Dano, K., Active outward transport of daunomycin in resistant Ehrlich ascites tumor cells. Biochim Biophys Acta, 1973. 323(3): p. 466-483.

2. Dean, M., The genetics of ATP-binding cassette transporters. Methods Enzymol, 2005. 400: p. 409-429.

3. Juliano, R.L. and Ling, V., A surface glycoprotein modulating drug permeability in Chinese hamster ovary cell mutants. Biochim Biophys Acta, 1976. 455(1): p. 152-162.

4. Kessel, D., Hall, T.C., Roberts, D., and Wodinsky, I., Uptake as a determinant of methotrexate response in mouse leukemias. Science, 1965. 150(697): p. 752-754.

5. Yesair, D.W., Kohner, F.A., Rogers, W.I., Baronowsky, P.E., and Kensler, C.J., Relationship of phthalanilide-lipid complexes to uptake and retention of 2-chloro4',4"-di(2-imidazolin-2-yl)terephthalanilide (NSC 60339) by sensitive and resistant P388 leukemia cells. Cancer Res, 1966. 26(2): p. 202-207.

6. Kessel, D., Botterill, V., and Wodinsky, I., Uptake and retention of daunomycin by mouse leukemic cells as factors in drug response. Cancer Res, 1968. 28(5): p. 938-941.

7. Riordan, J.R., Deuchars, K., Kartner, N., Alon, N., Trent, J., and Ling, V., Amplification of P-glycoprotein genes in multidrug-resistant mammalian cell lines. Nature, 1985. 316(6031): p. 817-819.

8. Roninson, I.B., Abelson, H.T., Housman, D.E., Howell, N., and Varshavsky, A., Amplification of specific DNA sequences correlates with multi-drug resistance in Chinese hamster cells. Nature, 1984. 309(5969): p. 626-628.

9. Gros, P., Croop, J., Roninson, I., Varshavsky, A., and Housman, D.E., Isolation and characterization of DNA sequences amplified in multidrug-resistant hamster cells. Proc Natl Acad Sci U S A, 1986. 83(2): p. 337-341.

10. Gros, P., Ben Neriah, Y.B., Croop, J.M., and Housman, D.E., Isolation and expression of a complementary DNA that confers multidrug resistance. Nature, 1986. 323(6090): p. 728-731.

11. Ueda, K., Cornwell, M.M., Gottesman, M.M., Pastan, I., Roninson, I.B., Ling, V., and Riordan, J.R., The mdrl gene, responsible for multidrug-resistance, codes for P-glycoprotein. Biochem Biophys Res Commun, 1986. 141(3): p. 956-962.

12. Bodzioch, M., Orso, E., Klucken, J., Langmann, T., Bottcher, A., Diederich, W., Drobnik, W., Barlage, S., Buchler, C., Porsch-Ozcurumez, M., Kaminski, W.E., Hahmann, H.W., Oette, K., Rothe, G., Aslanidis, C., Lackner, K.J., and Schmitz, G., The gene encoding ATP-binding cassette transporter 1 is mutated in Tangier disease. Nat Genet, 1999. 22(4): p. 347-351.

13. Brooks-Wilson, A., Marcil, M., Clee, S.M., Zhang, L.H., Roomp, K., van Dam, M., Yu, L., Brewer, C., Collins, J.A., Molhuizen, H.O., Loubser, O., Ouelette, B.F., Fichter, K., Ashbourne-Excoffon, K.J., Sensen, C.W., Scherer, S., Mott, S., Denis, M., Martindale, D., Frohlich, J., Morgan, K., Koop, B., Pimstone, S., Kastelein, J.J., Genest, J., Jr., and Hayden, M.R., Mutations in ABC1 in Tangier disease and familial high-density lipoprotein deficiency. Nat Genet, 1999. 22(4): p. 336-345. 
14. Marcil, M., Brooks-Wilson, A., Clee, S.M., Roomp, K., Zhang, L.H., Yu, L., Collins, J.A., van Dam, M., Molhuizen, H.O., Loubster, O., Ouellette, B.F., Sensen, C.W., Fichter, K., Mott, S., Denis, M., Boucher, B., Pimstone, S., Genest, J., Jr., Kastelein, J.J., and Hayden, M.R., Mutations in the ABC1 gene in familial HDL deficiency with defective cholesterol efflux. Lancet, 1999. 354(9187): $\mathrm{p}$. 1341-1346.

15. Remaley, A.T., Rust, S., Rosier, M., Knapper, C., Naudin, L., Broccardo, C., Peterson, K.M., Koch, C., Arnould, I., Prades, C., Duverger, N., Funke, H., Assman, G., Dinger, M., Dean, M., Chimini, G., Santamarina-Fojo, S., Fredrickson, D.S., Denefle, P., and Brewer, H.B., Jr., Human ATP-binding cassette transporter 1 (ABC1): genomic organization and identification of the genetic defect in the original Tangier disease kindred. Proc Natl Acad Sci U S A, 1999. 96(22): p. 12685-12690.

16. Rust, S., Rosier, M., Funke, H., Real, J., Amoura, Z., Piette, J.C., Deleuze, J.F., Brewer, H.B., Duverger, N., Denefle, P., and Assmann, G., Tangier disease is caused by mutations in the gene encoding ATP-binding cassette transporter 1. Nat Genet, 1999. 22(4): p. 352-355.

17. Shulenin, S., Nogee, L.M., Annilo, T., Wert, S.E., Whitsett, J.A., and Dean, M., ABCA3 gene mutations in newborns with fatal surfactant deficiency. $\mathrm{N}$ Engl $\mathrm{J}$ Med, 2004. 350(13): p. 1296-1303.

18. Allikmets, R., Shroyer, N.F., Singh, N., Seddon, J.M., Lewis, R.A., Bernstein, P.S., Peiffer, A., Zabriskie, N.A., Li, Y., Hutchinson, A., Dean, M., Lupski, J.R., and Leppert, M., Mutation of the Stargardt disease gene $(A B C R)$ in age-related macular degeneration. Science, 1997. 277(5333): p. 1805-1807.

19. Allikmets, R., Singh, N., Sun, H., Shroyer, N.F., Hutchinson, A., Chidambaram, A., Gerrard, B., Baird, L., Stauffer, D., Peiffer, A., Rattner, A., Smallwood, P., Li, Y., Anderson, K.L., Lewis, R.A., Nathans, J., Leppert, M., Dean, M., and Lupski, J.R., A photoreceptor cell-specific ATP-binding transporter gene (ABCR) is mutated in recessive Stargardt macular dystrophy. Nat Genet, 1997. 15(3): p. 236-246.

20. Lefevre, C., Audebert, S., Jobard, F., Bouadjar, B., Lakhdar, H., BoughdeneStambouli, O., Blanchet-Bardon, C., Heilig, R., Foglio, M., Weissenbach, J., Lathrop, M., Prud'homme, J.F., and Fischer, J., Mutations in the transporter ABCA12 are associated with lamellar ichthyosis type 2. Hum Mol Genet, 2003. 12(18): p. 2369-2378.

21. Schinkel, A.H., Smit, J.J., van Tellingen, O., Beijnen, J.H., Wagenaar, E., van Deemter, L., Mol, C.A., van der Valk, M.A., Robanus-Maandag, E.C., te Riele, H.P., and et al., Disruption of the mouse mdrla P-glycoprotein gene leads to a deficiency in the blood-brain barrier and to increased sensitivity to drugs. Cell, 1994. 77(4): p. 491-502.

22. Neff, M.W., Robertson, K.R., Wong, A.K., Safra, N., Broman, K.W., Slatkin, M., Mealey, K.L., and Pedersen, N.C., Breed distribution and history of canine mdr11Delta, a pharmacogenetic mutation that marks the emergence of breeds from the collie lineage. Proc Natl Acad Sci U S A, 2004. 101(32): p. 11725-11730. 
23. de la Salle, H., Zimmer, J., Fricker, D., Angenieux, C., Cazenave, J.P., Okubo, M., Maeda, H., Plebani, A., Tongio, M.M., Dormoy, A., and Hanau, D., HLA class I deficiencies due to mutations in subunit 1 of the peptide transporter TAPI. J Clin Invest, 1999. 103(5): p. R9-R13.

24. Teisserenc, H., Schmitt, W., Blake, N., Dunbar, R., Gadola, S., Gross, W.L., Exley, A., and Cerundolo, V., A case of primary immunodeficiency due to a defect of the major histocompatibility gene complex class I processing and presentation pathway. Immunol Lett, 1997. 57(1-3): p. 183-187.

25. Deleuze, J.F., Jacquemin, E., Dubuisson, C., Cresteil, D., Dumont, M., Erlinger, S., Bernard, O., and Hadchouel, M., Defect of multidrug-resistance 3 gene expression in a subtype of progressive familial intrahepatic cholestasis. Hepatology, 1996. 23(4): p. 904-908.

26. Allikmets, R., Raskind, W.H., Hutchinson, A., Schueck, N.D., Dean, M., and Koeller, D.M., Mutation of a putative mitochondrial iron transporter gene (ABC7) in X-linked sideroblastic anemia and ataxia (XLSA/A). Hum Mol Genet, 1999. 8(5): p. 743-749.

27. Strautnieks, S.S., Bull, L.N., Knisely, A.S., Kocoshis, S.A., Dahl, N., Arnell, H., Sokal, E., Dahan, K., Childs, S., Ling, V., Tanner, M.S., Kagalwalla, A.F., Nemeth, A., Pawlowska, J., Baker, A., Mieli-Vergani, G., Freimer, N.B., Gardiner, R.M., and Thompson, R.J., A gene encoding a liver-specific $A B C$ transporter is mutated in progressive familial intrahepatic cholestasis. Nat Genet, 1998. 20(3): p. 233-238.

28. Paulusma, C.C., Bosma, P.J., Zaman, G.J., Bakker, C.T., Otter, M., Scheffer, G.L., Scheper, R.J., Borst, P., and Oude Elferink, R.P., Congenital jaundice in rats with a mutation in a multidrug resistance-associated protein gene. Science, 1996. 271(5252): p. 1126-1128.

29. Bergen, A.A., Plomp, A.S., Schuurman, E.J., Terry, S., Breuning, M., Dauwerse, H., Swart, J., Kool, M., van Soest, S., Baas, F., ten Brink, J.B., and de Jong, P.T., Mutations in ABCC6 cause pseudoxanthoma elasticum. Nat Genet, 2000. 25(2): p. 228-231.

30. Riordan, J.R., Rommens, J.M., Kerem, B., Alon, N., Rozmahel, R., Grzelczak, Z., Zielenski, J., Lok, S., Plavsic, N., Chou, J.L., and et al., Identification of the cystic fibrosis gene: cloning and characterization of complementary DNA. Science, 1989. 245(4922): p. 1066-1073.

31. Thomas, P.M., Cote, G.J., Wohllk, N., Haddad, B., Mathew, P.M., Rabl, W., Aguilar-Bryan, L., Gagel, R.F., and Bryan, J., Mutations in the sulfonylurea receptor gene in familial persistent hyperinsulinemic hypoglycemia of infancy. Science, 1995. 268(5209): p. 426-429.

32. Bienengraeber, M., Olson, T.M., Selivanov, V.A., Kathmann, E.C., O'Cochlain, F., Gao, F., Karger, A.B., Ballew, J.D., Hodgson, D.M., Zingman, L.V., Pang, Y.P., Alekseev, A.E., and Terzic, A., ABCC9 mutations identified in human dilated cardiomyopathy disrupt catalytic KATP channel gating. Nat Genet, 2004. 36(4): p. 382-387. 
33. Yoshiura, K., Kinoshita, A., Ishida, T., Ninokata, A., Ishikawa, T., Kaname, T., Bannai, M., Tokunaga, K., Sonoda, S., Komaki, R., Ihara, M., Saenko, V.A., Alipov, G.K., Sekine, I., Komatsu, K., Takahashi, H., Nakashima, M., Sosonkina, N., Mapendano, C.K., Ghadami, M., Nomura, M., Liang, D.S., Miwa, N., Kim, D.K., Garidkhuu, A., Natsume, N., Ohta, T., Tomita, H., Kaneko, A., Kikuchi, M., Russomando, G., Hirayama, K., Ishibashi, M., Takahashi, A., Saitou, N., Murray, J.C., Saito, S., Nakamura, Y., and Niikawa, N., A SNP in the ABCC11 gene is the determinant of human earwax type. Nat Genet, 2006. 38(3): p. 324330.

34. Aubourg, P., Mosser, J., Douar, A.M., Sarde, C.O., Lopez, J., and Mandel, J.L., Adrenoleukodystrophy gene: unexpected homology to a protein involved in peroxisome biogenesis. Biochimie, 1993. 75(3-4): p. 293-302.

35. Jonker, J.W., Buitelaar, M., Wagenaar, E., Van Der Valk, M.A., Scheffer, G.L., Scheper, R.J., Plosch, T., Kuipers, F., Elferink, R.P., Rosing, H., Beijnen, J.H., and Schinkel, A.H., The breast cancer resistance protein protects against a major chlorophyll-derived dietary phototoxin and protoporphyria. Proc Natl Acad Sci U S A, 2002. 99(24): p. 15649-15654.

36. Berge, K.E., Tian, H., Graf, G.A., Yu, L., Grishin, N.V., Schultz, J., Kwiterovich, P., Shan, B., Barnes, R., and Hobbs, H.H., Accumulation of dietary cholesterol in sitosterolemia caused by mutations in adjacent ABC transporters. Science, 2000. 290(5497): p. 1771-1775.

37. Walker, J.E., Saraste, M., Runswick, M.J., and Gay, N.J., Distantly related sequences in the alpha- and beta-subunits of ATP synthase, myosin, kinases and other ATP-requiring enzymes and a common nucleotide binding fold. EMBO J, 1982. 1(8): p. 945-951.

38. Higgins, C.F., Hiles, I.D., Whalley, K., and Jamieson, D.J., Nucleotide binding by membrane components of bacterial periplasmic binding protein-dependent transport systems. EMBO J, 1985. 4(4): p. 1033-1039.

39. Gros, P., Croop, J., and Housman, D., Mammalian multidrug resistance gene: complete cDNA sequence indicates strong homology to bacterial transport proteins. Cell, 1986. 47(3): p. 371-380.

40. Chen, C.J., Chin, J.E., Ueda, K., Clark, D.P., Pastan, I., Gottesman, M.M., and Roninson, I.B., Internal duplication and homology with bacterial transport proteins in the mdrl (P-glycoprotein) gene from multidrug-resistant human cells. Cell, 1986. 47(3): p. 381-389.

41. Gerlach, J.H., Endicott, J.A., Juranka, P.F., Henderson, G., Sarangi, F., Deuchars, K.L., and Ling, V., Homology between P-glycoprotein and a bacterial haemolysin transport protein suggests a model for multidrug resistance. Nature, 1986.

324(6096): p. 485-489.

42. Higgins, C.F., Hiles, I.D., Salmond, G.P., Gill, D.R., Downie, J.A., Evans, I.J., Holland, I.B., Gray, L., Buckel, S.D., Bell, A.W., and et al., A family of related ATP-binding subunits coupled to many distinct biological processes in bacteria. Nature, 1986. 323(6087): p. 448-450. 
43. Hyde, S.C., Emsley, P., Hartshorn, M.J., Mimmack, M.M., Gileadi, U., Pearce, S.R., Gallagher, M.P., Gill, D.R., Hubbard, R.E., and Higgins, C.F., Structural model of ATP-binding proteins associated with cystic fibrosis, multidrug resistance and bacterial transport. Nature, 1990. 346(6282): p. 362-365.

44. Allikmets, R., Gerrard, B., Hutchinson, A., and Dean, M., Characterization of the human ABC superfamily: isolation and mapping of 21 new genes using the expressed sequence tags database. Hum Mol Genet, 1996. 5(10): p. 1649-1655.

45. Rottenberg, S., Nygren, A.O., Pajic, M., van Leeuwen, F.W., van der Heijden, I., van de Wetering, K., Liu, X., de Visser, K.E., Gilhuijs, K.G., van Tellingen, O., Schouten, J.P., Jonkers, J., and Borst, P., Selective induction of chemotherapy resistance of mammary tumors in a conditional mouse model for hereditary breast cancer. Proc Natl Acad Sci U S A, 2007. 104(29): p. 12117-12122.

46. Cole, S.P., Bhardwaj, G., Gerlach, J.H., Mackie, J.E., Grant, C.E., Almquist, K.C., Stewart, A.J., Kurz, E.U., Duncan, A.M., and Deeley, R.G., Overexpression of a transporter gene in a multidrug-resistant human lung cancer cell line.

Science, 1992. 258(5088): p. 1650-1654.

47. Doyle, L.A., Yang, W., Abruzzo, L.V., Krogmann, T., Gao, Y., Rishi, A.K., and Ross, D.D., A multidrug resistance transporter from human MCF-7 breast cancer cells. Proc Natl Acad Sci U S A, 1998. 95(26): p. 15665-15670.

48. Allikmets, R., Schriml, L.M., Hutchinson, A., Romano-Spica, V., and Dean, M., A human placenta-specific ATP-binding cassette gene (ABCP) on chromosome $4 q 22$ that is involved in multidrug resistance. Cancer Res, 1998. 58(23): p. 53375339.

49. Miyake, K., Mickley, L., Litman, T., Zhan, Z., Robey, R., Cristensen, B., Brangi, M., Greenberger, L., Dean, M., Fojo, T., and Bates, S.E., Molecular cloning of cDNAs which are highly overexpressed in mitoxantrone-resistant cells: demonstration of homology to ABC transport genes. Cancer Res, 1999. 59(1): p. 8-13.

50. Szakacs, G., Varadi, A., Ozvegy-Laczka, C., and Sarkadi, B., The role of $A B C$ transporters in drug absorption, distribution, metabolism, excretion and toxicity (ADME-Tox). Drug Discov Today, 2008. 13(9-10): p. 379-393.

51. Kool, M., de Haas, M., Scheffer, G.L., Scheper, R.J., van Eijk, M.J., Juijn, J.A., Baas, F., and Borst, P., Analysis of expression of cMOAT (MRP2), MRP3, MRP4, and MRP5, homologues of the multidrug resistance-associated protein gene (MRP1), in human cancer cell lines. Cancer Res, 1997. 57(16): p. 3537-3547.

52. Schuetz, J.D., Connelly, M.C., Sun, D., Paibir, S.G., Flynn, P.M., Srinivas, R.V., Kumar, A., and Fridland, A., MRP4: A previously unidentified factor in resistance to nucleoside-based antiviral drugs. Nat Med, 1999. 5(9): p. 1048-1051.

53. Szakacs, G., Paterson, J.K., Ludwig, J.A., Booth-Genthe, C., and Gottesman, M.M., Targeting multidrug resistance in cancer. Nat Rev Drug Discov, 2006. 5(3): p. 219-234.

54. Takenaka, K., Morgan, J.A., Scheffer, G.L., Adachi, M., Stewart, C.F., Sun, D., Leggas, M., Ejendal, K.F., Hrycyna, C.A., and Schuetz, J.D., Substrate overlap between Mrp4 and Abcg2/Bcrp affects purine analogue drug cytotoxicity and tissue distribution. Cancer Res, 2007. 67(14): p. 6965-6972. 
55. Cascorbi, I., Role of pharmacogenetics of ATP-binding cassette transporters in the pharmacokinetics of drugs. Pharmacol Ther, 2006. 112(2): p. 457-473.

56. Thiebaut, F., Tsuruo, T., Hamada, H., Gottesman, M.M., Pastan, I., and Willingham, M.C., Cellular localization of the multidrug-resistance gene product P-glycoprotein in normal human tissues. Proc Natl Acad Sci U S A, 1987. 84(21): p. 7735-7738.

57. Graf, G.A., Yu, L., Li, W.P., Gerard, R., Tuma, P.L., Cohen, J.C., and Hobbs, H.H., ABCG5 and ABCG8 are obligate heterodimers for protein trafficking and biliary cholesterol excretion. J Biol Chem, 2003. 278(48): p. 48275-48282.

58. Wang, R., Salem, M., Yousef, I.M., Tuchweber, B., Lam, P., Childs, S.J., Helgason, C.D., Ackerley, C., Phillips, M.J., and Ling, V., Targeted inactivation of sister of P-glycoprotein gene (spgp) in mice results in nonprogressive but persistent intrahepatic cholestasis. Proc Natl Acad Sci U S A, 2001. 98(4): p. 2011-2016.

59. Smit, J.J., Schinkel, A.H., Oude Elferink, R.P., Groen, A.K., Wagenaar, E., van Deemter, L., Mol, C.A., Ottenhoff, R., van der Lugt, N.M., van Roon, M.A., and et al., Homozygous disruption of the murine mdr2 P-glycoprotein gene leads to a complete absence of phospholipid from bile and to liver disease. Cell, 1993. 75(3): p. 451-462.

60. Konig, J., Rost, D., Cui, Y., and Keppler, D., Characterization of the human multidrug resistance protein isoform MRP3 localized to the basolateral hepatocyte membrane. Hepatology, 1999. 29(4): p. 1156-1163.

61. Rius, M., Nies, A.T., Hummel-Eisenbeiss, J., Jedlitschky, G., and Keppler, D., Cotransport of reduced glutathione with bile salts by MRP4 (ABCC4) localized to the basolateral hepatocyte membrane. Hepatology, 2003. 38(2): p. 374-384.

62. Scheffer, G.L., Hu, X., Pijnenborg, A.C., Wijnholds, J., Bergen, A.A., and Scheper, R.J., MRP6 (ABCC6) detection in normal human tissues and tumors. Lab Invest, 2002. 82(4): p. 515-518.

63. Langheim, S., Yu, L., von Bergmann, K., Lutjohann, D., Xu, F., Hobbs, H.H., and Cohen, J.C., ABCG5 and ABCG8 require MDR2 for secretion of cholesterol into bile. J Lipid Res, 2005. 46(8): p. 1732-1738.

64. Wang, J., Sun, F., Zhang, D.W., Ma, Y., Xu, F., Belani, J.D., Cohen, J.C., Hobbs, H.H., and Xie, X.S., Sterol transfer by ABCG5 and ABCG8: in vitro assay and reconstitution. J Biol Chem, 2006. 281(38): p. 27894-27904.

65. Hirohashi, T., Suzuki, H., Takikawa, H., and Sugiyama, Y., ATP-dependent transport of bile salts by rat multidrug resistance-associated protein 3 (Mrp3). J Biol Chem, 2000. 275(4): p. 2905-2910.

66. Potschka, H., Fedrowitz, M., and Loscher, W., Multidrug resistance protein MRP2 contributes to blood-brain barrier function and restricts antiepileptic drug activity. J Pharmacol Exp Ther, 2003. 306(1): p. 124-131.

67. Leggas, M., Adachi, M., Scheffer, G.L., Sun, D., Wielinga, P., Du, G., Mercer, K.E., Zhuang, Y., Panetta, J.C., Johnston, B., Scheper, R.J., Stewart, C.F., and Schuetz, J.D., Mrp4 confers resistance to topotecan and protects the brain from chemotherapy. Mol Cell Biol, 2004. 24(17): p. 7612-7621. 
68. Nies, A.T., Jedlitschky, G., Konig, J., Herold-Mende, C., Steiner, H.H., Schmitt, H.P., and Keppler, D., Expression and immunolocalization of the multidrug resistance proteins, MRP1-MRP6 (ABCC1-ABCC6), in human brain.

Neuroscience, 2004. 129(2): p. 349-360.

69. Cooray, H.C., Blackmore, C.G., Maskell, L., and Barrand, M.A., Localisation of breast cancer resistance protein in microvessel endothelium of human brain.

Neuroreport, 2002. 13(16): p. 2059-2063.

70. Wijnholds, J., deLange, E.C., Scheffer, G.L., van den Berg, D.J., Mol, C.A., van der Valk, M., Schinkel, A.H., Scheper, R.J., Breimer, D.D., and Borst, P., Multidrug resistance protein 1 protects the choroid plexus epithelium and contributes to the blood-cerebrospinal fluid barrier. J Clin Invest, 2000. 105(3): p. 279-285.

71. Rao, V.V., Dahlheimer, J.L., Bardgett, M.E., Snyder, A.Z., Finch, R.A., Sartorelli, A.C., and Piwnica-Worms, D., Choroid plexus epithelial expression of MDR 1 P glycoprotein and multidrug resistance-associated protein contribute to the blood-cerebrospinal-fluid drug-permeability barrier. Proc Natl Acad Sci U S A, 1999. 96(7): p. 3900-3905.

72. Smit, J.W., Huisman, M.T., van Tellingen, O., Wiltshire, H.R., and Schinkel, A.H., Absence or pharmacological blocking of placental P-glycoprotein profoundly increases fetal drug exposure. J Clin Invest, 1999. 104(10): p. 14411447.

73. Chaudhary, P.M. and Roninson, I.B., Expression and activity of P-glycoprotein, a multidrug efflux pump, in human hematopoietic stem cells. Cell, 1991. 66(1): p. 85-94.

74. Zhou, S., Schuetz, J.D., Bunting, K.D., Colapietro, A.M., Sampath, J., Morris, J.J., Lagutina, I., Grosveld, G.C., Osawa, M., Nakauchi, H., and Sorrentino, B.P., The ABC transporter Bcrp1/ABCG2 is expressed in a wide variety of stem cells and is a molecular determinant of the side-population phenotype. Nat Med, 2001. 7(9): p. 1028-1034.

75. Krishnamurthy, P., Ross, D.D., Nakanishi, T., Bailey-Dell, K., Zhou, S., Mercer, K.E., Sarkadi, B., Sorrentino, B.P., and Schuetz, J.D., The stem cell marker Bcrp/ABCG2 enhances hypoxic cell survival through interactions with heme. $\mathrm{J}$ Biol Chem, 2004. 279(23): p. 24218-24225.

76. Powis, S.J., Townsend, A.R., Deverson, E.V., Bastin, J., Butcher, G.W., and Howard, J.C., Restoration of antigen presentation to the mutant cell line RMA-S by an MHC-linked transporter. Nature, 1991. 354(6354): p. 528-531.

77. Spies, T. and DeMars, R., Restored expression of major histocompatibility class I molecules by gene transfer of a putative peptide transporter. Nature, 1991. 351(6324): p. 323-324.

78. Androlewicz, M.J., Anderson, K.S., and Cresswell, P., Evidence that transporters associated with antigen processing translocate a major histocompatibility complex class I-binding peptide into the endoplasmic reticulum in an ATPdependent manner. Proc Natl Acad Sci U S A, 1993. 90(19): p. 9130-9134.

79. Zhang, F., Zhang, W., Liu, L., Fisher, C.L., Hui, D., Childs, S., Dorovini-Zis, K., and Ling, V., Characterization of $A B C B 9$, an ATP binding cassette protein associated with lysosomes. J Biol Chem, 2000. 275(30): p. 23287-23294. 
80. Wolters, J.C., Abele, R., and Tampe, R., Selective and ATP-dependent translocation of peptides by the homodimeric ATP binding cassette transporter TAP-like (ABCB9). J Biol Chem, 2005. 280(25): p. 23631-23636.

81. Wanders, R.J., Visser, W.F., van Roermund, C.W., Kemp, S., and Waterham, H.R., The peroxisomal ABC transporter family. Pflugers Arch, 2007. 453(5): p. 719-734.

82. Bear, C.E., Li, C.H., Kartner, N., Bridges, R.J., Jensen, T.J., Ramjeesingh, M., and Riordan, J.R., Purification and functional reconstitution of the cystic fibrosis transmembrane conductance regulator (CFTR). Cell, 1992. 68(4): p. 809-818.

83. Bryan, J., Munoz, A., Zhang, X., Dufer, M., Drews, G., Krippeit-Drews, P., and Aguilar-Bryan, L., $A B C C 8$ and ABCC9: $A B C$ transporters that regulate $K^{+}$ channels. Pflugers Arch, 2007. 453(5): p. 703-718.

84. Cheng, S.H., Gregory, R.J., Marshall, J., Paul, S., Souza, D.W., White, G.A., O'Riordan, C.R., and Smith, A.E., Defective intracellular transport and processing of CFTR is the molecular basis of most cystic fibrosis. Cell, 1990. 63(4): p. 827-834.

85. Mealey, K.L., Bentjen, S.A., Gay, J.M., and Cantor, G.H., Ivermectin sensitivity in collies is associated with a deletion mutation of the mdrl gene.

Pharmacogenetics, 2001. 11(8): p. 727-733.

86. Guo, Y., Kotova, E., Chen, Z.S., Lee, K., Hopper-Borge, E., Belinsky, M.G., and Kruh, G.D., MRP8, ATP-binding cassette C11 (ABCC11), is a cyclic nucleotide efflux pump and a resistance factor for fluoropyrimidines 2',3'-dideoxycytidine and 9'-(2'-phosphonylmethoxyethyl)adenine. J Biol Chem, 2003. 278(32): p. 29509-29514.

87. Koch, J., Guntrum, R., and Tampe, R., Exploring the minimal functional unit of the transporter associated with antigen processing. FEBS Lett, 2005. 579(20): p. 4413-4416.

88. Hrycyna, C.A., Ramachandra, M., Germann, U.A., Cheng, P.W., Pastan, I., and Gottesman, M.M., Both ATP sites of human P-glycoprotein are essential but not symmetric. Biochemistry, 1999. 38(42): p. 13887-13899.

89. Berkower, C. and Michaelis, S., Mutational analysis of the yeast a-factor transporter STE6, a member of the ATP binding cassette (ABC) protein superfamily. EMBO J, 1991. 10(12): p. 3777-3785.

90. Loo, T.W. and Clarke, D.M., Reconstitution of drug-stimulated ATPase activity following co-expression of each half of human P-glycoprotein as separate polypeptides. J Biol Chem, 1994. 269(10): p. 7750-7755.

91. Xu, J., Liu, Y., Yang, Y., Bates, S., and Zhang, J.T., Characterization of oligomeric human half-ABC transporter ATP-binding cassette G2. J Biol Chem, 2004. 279(19): p. 19781-19789.

92. Kelly, A., Powis, S.H., Kerr, L.A., Mockridge, I., Elliott, T., Bastin, J., UchanskaZiegler, B., Ziegler, A., Trowsdale, J., and Townsend, A., Assembly and function of the two ABC transporter proteins encoded in the human major histocompatibility complex. Nature, 1992. 355(6361): p. 641-644. 
93. Graf, G.A., Li, W.P., Gerard, R.D., Gelissen, I., White, A., Cohen, J.C., and Hobbs, H.H., Coexpression of ATP-binding cassette proteins ABCG5 and ABCG8 permits their transport to the apical surface. J Clin Invest, 2002. 110(5): p. 659669.

94. Chloupkova, M., Reaves, S.K., LeBard, L.M., and Koeller, D.M., The mitochondrial ABC transporter Atm 1p functions as a homodimer. FEBS Lett, 2004. 569(1-3): p. 65-69.

95. Polgar, O., Robey, R.W., Morisaki, K., Dean, M., Michejda, C., Sauna, Z.E., Ambudkar, S.V., Tarasova, N., and Bates, S.E., Mutational analysis of ABCG2: role of the GXXXG motif. Biochemistry, 2004. 43(29): p. 9448-9456.

96. Xu, J., Peng, H., Chen, Q., Liu, Y., Dong, Z., and Zhang, J.T., Oligomerization domain of the multidrug resistance-associated transporter ABCG2 and its dominant inhibitory activity. Cancer Res, 2007. 67(9): p. 4373-4381.

97. Dawson, R.J. and Locher, K.P., Structure of a bacterial multidrug ABC transporter. Nature, 2006. 443(7108): p. 180-185.

98. Stenham, D.R., Campbell, J.D., Sansom, M.S., Higgins, C.F., Kerr, I.D., and Linton, K.J., An atomic detail model for the human ATP binding cassette transporter P-glycoprotein derived from disulfide cross-linking and homology modeling. FASEB J, 2003. 17(15): p. 2287-2289.

99. Schrodt, S., Koch, J., and Tampe, R., Membrane topology of the transporter associated with antigen processing (TAP1) within an assembled functional peptide-loading complex. J Biol Chem, 2006. 281(10): p. 6455-6462.

100. Gao, M., Yamazaki, M., Loe, D.W., Westlake, C.J., Grant, C.E., Cole, S.P., and Deeley, R.G., Multidrug resistance protein. Identification of regions required for active transport of leukotriene C4. J Biol Chem, 1998. 273(17): p. 10733-10740.

101. Koch, J., Guntrum, R., and Tampe, R., The first N-terminal transmembrane helix of each subunit of the antigenic peptide transporter TAP is essential for independent tapasin binding. FEBS Lett, 2006. 580(17): p. 4091-4096.

102. Chen, Q., Yang, Y., Li, L., and Zhang, J.T., The amino terminus of the human multidrug resistance transporter ABCC1 has a U-shaped folding with a gating function. J Biol Chem, 2006. 281(41): p. 31152-31163.

103. Tamai, I. and Safa, A.R., Azidopine noncompetitively interacts with vinblastine and cyclosporin A binding to P-glycoprotein in multidrug resistant cells. J Biol Chem, 1991. 266(25): p. 16796-16800.

104. Loo, T.W., Bartlett, M.C., and Clarke, D.M., Methanethiosulfonate derivatives of rhodamine and verapamil activate human P-glycoprotein at different sites. J Biol Chem, 2003. 278(50): p. 50136-50141.

105. Schumacher, M.A. and Brennan, R.G., Deciphering the molecular basis of multidrug recognition: crystal structures of the Staphylococcus aureus multidrug binding transcription regulator QacR. Res Microbiol, 2003. 154(2): p. 69-77.

106. Schumacher, M.A., Miller, M.C., and Brennan, R.G., Structural mechanism of the simultaneous binding of two drugs to a multidrug-binding protein. EMBO J, 2004. 23(15): p. 2923-2930. 
107. Clark, R., Kerr, I.D., and Callaghan, R., Multiple drugbinding sites on the R482G isoform of the ABCG2 transporter. Br J Pharmacol, 2006. 149(5): p. 506-515.

108. Deeley, R.G. and Cole, S.P., Substrate recognition and transport by multidrug resistance protein 1 (ABCC1). FEBS Lett, 2006. 580(4): p. 1103-1111.

109. Greenberger, L.M., Major photoaffinity drug labeling sites for iodoaryl azidoprazosin in P-glycoprotein are within, or immediately $C$-terminal to, transmembrane domains 6 and 12. J Biol Chem, 1993. 268(15): p. 11417-11425.

110. Smith, P.C., Karpowich, N., Millen, L., Moody, J.E., Rosen, J., Thomas, P.J., and Hunt, J.F., ATP binding to the motor domain from an ABC transporter drives formation of a nucleotide sandwich dimer. Mol Cell, 2002. 10(1): p. 139-149.

111. Procko, E., Ferrin-O'Connell, I., Ng, S.L., and Gaudet, R., Distinct structural and functional properties of the ATPase sites in an asymmetric ABC transporter. Mol Cell, 2006. 24(1): p. 51-62.

112. Gaudet, R. and Wiley, D.C., Structure of the ABC ATPase domain of human TAP1, the transporter associated with antigen processing. EMBO J, 2001. 20(17): p. 4964-4972.

113. Senior, A.E., al-Shawi, M.K., and Urbatsch, I.L., The catalytic cycle of Pglycoprotein. FEBS Lett, 1995. 377(3): p. 285-289.

114. Higgins, C.F. and Linton, K.J., The ATP switch model for ABC transporters. Nat Struct Mol Biol, 2004. 11(10): p. 918-926.

115. Wang, G., Pincheira, R., and Zhang, J.T., Dissection of drug-binding-induced conformational changes in P-glycoprotein. Eur J Biochem, 1998. 255(2): p. 383390.

116. Urquhart, B.L., Tirona, R.G., and Kim, R.B., Nuclear receptors and the regulation of drug-metabolizing enzymes and drug transporters: implications for interindividual variability in response to drugs. J Clin Pharmacol, 2007. 47(5): p. 566-578.

117. Sinal, C.J., Tohkin, M., Miyata, M., Ward, J.M., Lambert, G., and Gonzalez, F.J., Targeted disruption of the nuclear receptor FXR/BAR impairs bile acid and lipid homeostasis. Cell, 2000. 102(6): p. 731-744.

118. Ananthanarayanan, M., Balasubramanian, N., Makishima, M., Mangelsdorf, D.J., and Suchy, F.J., Human bile salt export pump promoter is transactivated by the farnesoid X receptor/bile acid receptor. J Biol Chem, 2001. 276(31): p. 2885728865.

119. Assem, M., Schuetz, E.G., Leggas, M., Sun, D., Yasuda, K., Reid, G., Zelcer, N., Adachi, M., Strom, S., Evans, R.M., Moore, D.D., Borst, P., and Schuetz, J.D., Interactions between hepatic Mrp4 and Sult2a as revealed by the constitutive androstane receptor and Mrp4 knockout mice. J Biol Chem, 2004. 279(21): p. 22250-22257.

120. Turner, J.G., Gump, J.L., Zhang, C., Cook, J.M., Marchion, D., Hazlehurst, L., Munster, P., Schell, M.J., Dalton, W.S., and Sullivan, D.M., ABCG2 expression, function, and promoter methylation in human multiple myeloma. Blood, 2006. 108(12): p. 3881-3889. 
121. Calcagno, A.M., Fostel, J.M., To, K.K., Salcido, C.D., Martin, S.E., Chewning, K.J., Wu, C.P., Varticovski, L., Bates, S.E., Caplen, N.J., and Ambudkar, S.V., Single-step doxorubicin-selected cancer cells overexpress the ABCG2 drug transporter through epigenetic changes. Br J Cancer, 2008. 98(9): p. 1515-1524.

122. Nakano, H., Nakamura, Y., Soda, H., Kamikatahira, M., Uchida, K., Takasu, M., Kitazaki, T., Yamaguchi, H., Nakatomi, K., Yanagihara, K., Kohno, S., and Tsukamoto, K., Methylation status of breast cancer resistance protein detected by methylation-specific polymerase chain reaction analysis is correlated inversely with its expression in drug-resistant lung cancer cells. Cancer, 2008. 112(5): $\mathrm{p}$. 1122-1130.

123. To, K.K., Polgar, O., Huff, L.M., Morisaki, K., and Bates, S.E., Histone modifications at the ABCG2 promoter following treatment with histone deacetylase inhibitor mirror those in multidrug-resistant cells. Mol Cancer Res, 2008. 6(1): p. 151-164.

124. Nakanishi, T., Bailey-Dell, K.J., Hassel, B.A., Shiozawa, K., Sullivan, D.M., Turner, J., and Ross, D.D., Novel 5' untranslated region variants of BCRP $m R N A$ are differentially expressed in drug-selected cancer cells and in normal human tissues: implications for drug resistance, tissue-specific expression, and alternative promoter usage. Cancer Res, 2006. 66(10): p. 5007-5011.

125. Zong, Y., Zhou, S., Fatima, S., and Sorrentino, B.P., Expression of mouse Abcg2 $m R N A$ during hematopoiesis is regulated by alternative use of multiple leader exons and promoters. J Biol Chem, 2006. 281(40): p. 29625-29632.

126. Poonkuzhali, B., Lamba, J., Strom, S., Sparreboom, A., Thummel, K., Watkins, P., and Schuetz, E., Association of breast cancer resistance protein/ABCG2 phenotypes and novel promoter and intron 1 single nucleotide polymorphisms. Drug Metab Dispos, 2008. 36(4): p. 780-795.

127. Grant, C.E., Kurz, E.U., Cole, S.P., and Deeley, R.G., Analysis of the intron-exon organization of the human multidrug-resistance protein gene (MRP) and alternative splicing of its $m R N A$. Genomics, 1997. 45(2): p. 368-378.

128. Suzuki, T., Sasaki, H., Kuh, H.J., Agui, M., Tatsumi, Y., Tanabe, S., Terada, M., Saijo, N., and Nishio, K., Detailed structural analysis on both human MRP5 and mouse mrp5 transcripts. Gene, 2000. 242(1-2): p. 167-173.

129. Lamba, J.K., Adachi, M., Sun, D., Tammur, J., Schuetz, E.G., Allikmets, R., and Schuetz, J.D., Nonsense mediated decay downregulates conserved alternatively spliced ABCC4 transcripts bearing nonsense codons. Hum Mol Genet, 2003. 12(2): p. 99-109.

130. Stojic, J., Stohr, H., and Weber, B.H., Three novel ABCC5 splice variants in human retina and their role as regulators of ABCC5 gene expression. BMC Mol Biol, 2007. 8: p. 42.

131. Kimchi-Sarfaty, C., Oh, J.M., Kim, I.W., Sauna, Z.E., Calcagno, A.M., Ambudkar, S.V., and Gottesman, M.M., $A$ "silent" polymorphism in the MDRI gene changes substrate specificity. Science, 2007.315(5811): p. 525-528.

132. Schinkel, A.H., Kemp, S., Dolle, M., Rudenko, G., and Wagenaar, E., $N$ glycosylation and deletion mutants of the human MDRI P-glycoprotein. J Biol Chem, 1993. 268(10): p. 7474-7481. 
133. Mochizuki, K., Kagawa, T., Numari, A., Harris, M.J., Itoh, J., Watanabe, N., Mine, T., and Arias, I.M., Two $N$-linked glycans are required to maintain the transport activity of the bile salt export pump (ABCB11) in MDCK II cells. Am J Physiol Gastrointest Liver Physiol, 2007. 292(3): p. G818-828.

134. Diop, N.K. and Hrycyna, C.A., N-Linked glycosylation of the human ABC transporter ABCG2 on asparagine 596 is not essential for expression, transport activity, or trafficking to the plasma membrane. Biochemistry, 2005. 44(14): p. 5420-5429.

135. Li, C. and Naren, A.P., Macromolecular complexes of cystic fibrosis transmembrane conductance regulator and its interacting partners. Pharmacol Ther, 2005. 108(2): p. 208-223.

136. Li, C., Dandridge, K.S., Di, A., Marrs, K.L., Harris, E.L., Roy, K., Jackson, J.S., Makarova, N.V., Fujiwara, Y., Farrar, P.L., Nelson, D.J., Tigyi, G.J., and Naren, A.P., Lysophosphatidic acid inhibits cholera toxin-induced secretory diarrhea through CFTR-dependent protein interactions. J Exp Med, 2005. 202(7): p. 975986.

137. Li, C., Krishnamurthy, P.C., Penmatsa, H., Marrs, K.L., Wang, X.Q., Zaccolo, M., Jalink, K., Li, M., Nelson, D.J., Schuetz, J.D., and Naren, A.P., Spatiotemporal coupling of cAMP transporter to CFTR chloride channel function in the gut epithelia. Cell, 2007. 131(5): p. 940-951.

138. Herget, M. and Tampe, R., Intracellular peptide transporters in human-compartmentalization of the "peptidome". Pflugers Arch, 2007. 453(5): p. 591600.

139. van Endert, P.M., Tampe, R., Meyer, T.H., Tisch, R., Bach, J.F., and McDevitt, H.O., A sequential model for peptide binding and transport by the transporters associated with antigen processing. Immunity, 1994. 1(6): p. 491-500.

140. Knittler, M.R., Alberts, P., Deverson, E.V., and Howard, J.C., Nucleotide binding by TAP mediates association with peptide and release of assembled MHC class I molecules. Curr Biol, 1999. 9(18): p. 999-1008.

141. Alberts, P., Daumke, O., Deverson, E.V., Howard, J.C., and Knittler, M.R., Distinct functional properties of the TAP subunits coordinate the nucleotidedependent transport cycle. Curr Biol, 2001. 11(4): p. 242-251.

142. Gorbulev, S., Abele, R., and Tampe, R., Allosteric crosstalk between peptidebinding, transport, and ATP hydrolysis of the ABC transporter TAP. Proc Natl Acad Sci U S A, 2001. 98(7): p. 3732-3737.

143. Karttunen, J.T., Lehner, P.J., Gupta, S.S., Hewitt, E.W., and Cresswell, P., Distinct functions and cooperative interaction of the subunits of the transporter associated with antigen processing (TAP). Proc Natl Acad Sci U S A, 2001. 98(13): p. 7431-7436.

144. Bouabe, H. and Knittler, M.R., The distinct nucleotide binding states of the transporter associated with antigen processing (TAP) are regulated by the nonhomologous C-terminal tails of TAP1 and TAP2. Eur J Biochem, 2003. 270(22): p. 4531-4546.

145. Yamaguchi, Y., Kasano, M., Terada, T., Sato, R., and Maeda, M., An ABC transporter homologous to TAP proteins. FEBS Lett, 1999. 457(2): p. 231-236. 
146. Demirel, O., Waibler, Z., Kalinke, U., Grunebach, F., Appel, S., Brossart, P., Hasilik, A., Tampe, R., and Abele, R., Identification of a lysosomal peptide transport system induced during dendritic cell development. J Biol Chem, 2007. 282(52): p. 37836-37843.

147. Leveson-Gower, D.B., Michnick, S.W., and Ling, V., Detection of TAP family dimerizations by an in vivo assay in mammalian cells. Biochemistry, 2004. 43(44): p. 14257-14264.

148. Hogue, D.L., Liu, L., and Ling, V., Identification and characterization of a mammalian mitochondrial ATP-binding cassette membrane protein. J Mol Biol, 1999. 285(1): p. 379-389.

149. Ardehali, H., O'Rourke, B., and Marban, E., Cardioprotective role of the mitochondrial ATP-binding cassette protein 1. Circ Res, 2005. 97(8): p. 740-742.

150. Ardehali, H., Chen, Z., Ko, Y., Mejia-Alvarez, R., and Marban, E., Multiprotein complex containing succinate dehydrogenase confers mitochondrial ATPsensitive $K+$ channel activity. Proc Natl Acad Sci U S A, 2004. 101(32): p. $11880-11885$.

151. Leighton, J. and Schatz, G., An ABC transporter in the mitochondrial inner membrane is required for normal growth of yeast. EMBO J, 1995. 14(1): p. 188195.

152. Kispal, G., Csere, P., Guiard, B., and Lill, R., The ABC transporter Atm 1p is required for mitochondrial iron homeostasis. FEBS Lett, 1997. 418(3): p. 346350.

153. Senbongi, H., Ling, F., and Shibata, T., A mutation in a mitochondrial ABC transporter results in mitochondrial dysfunction through oxidative damage of mitochondrial DNA. Mol Gen Genet, 1999. 262(3): p. 426-436.

154. Leighton, J., ATP-binding cassette transporter in Saccharomyces cerevisiae mitochondria. Methods Enzymol, 1995. 260: p. 389-396.

155. Kispal, G., Csere, P., Prohl, C., and Lill, R., The mitochondrial proteins Atm 1p and Nfs $1 p$ are essential for biogenesis of cytosolic Fe/S proteins. EMBO J, 1999. 18(14): p. 3981-3989.

156. Lill, R. and Muhlenhoff, U., Maturation of iron-sulfur proteins in eukaryotes: mechanisms, connected processes, and diseases. Annu Rev Biochem, 2008. 77: p. 669-700.

157. Chloupkova, M., LeBard, L.S., and Koeller, D.M., MDL1 is a high copy suppressor of ATM1: evidence for a role in resistance to oxidative stress. J Mol Biol, 2003. 331(1): p. 155-165.

158. Wingert, R.A., Galloway, J.L., Barut, B., Foott, H., Fraenkel, P., Axe, J.L., Weber, G.J., Dooley, K., Davidson, A.J., Schmid, B., Paw, B.H., Shaw, G.C., Kingsley, P., Palis, J., Schubert, H., Chen, O., Kaplan, J., and Zon, L.I., Deficiency of glutaredoxin 5 reveals $\mathrm{Fe}-\mathrm{S}$ clusters are required for vertebrate haem synthesis. Nature, 2005. 436(7053): p. 1035-1039.

159. Csere, P., Lill, R., and Kispal, G., Identification of a human mitochondrial ABC transporter, the functional orthologue of yeast Atm 1p. FEBS Lett, 1998. 441(2): p. 266-270. 
160. Bekri, S., Kispal, G., Lange, H., Fitzsimons, E., Tolmie, J., Lill, R., and Bishop, D.F., Human ABC7 transporter: gene structure and mutation causing X-linked sideroblastic anemia with ataxia with disruption of cytosolic iron-sulfur protein maturation. Blood, 2000. 96(9): p. 3256-3264.

161. Pondarre, C., Campagna, D.R., Antiochos, B., Sikorski, L., Mulhern, H., and Fleming, M.D., Abcb7, the gene responsible for X-linked sideroblastic anemia with ataxia, is essential for hematopoiesis. Blood, 2007. 109(8): p. 3567-3569.

162. Pondarre, C., Antiochos, B.B., Campagna, D.R., Clarke, S.L., Greer, E.L., Deck, K.M., McDonald, A., Han, A.P., Medlock, A., Kutok, J.L., Anderson, S.A., Eisenstein, R.S., and Fleming, M.D., The mitochondrial ATP-binding cassette transporter Abcb7 is essential in mice and participates in cytosolic iron-sulfur cluster biogenesis. Hum Mol Genet, 2006. 15(6): p. 953-964.

163. Cavadini, P., Biasiotto, G., Poli, M., Levi, S., Verardi, R., Zanella, I., Derosas, M., Ingrassia, R., Corrado, M., and Arosio, P., RNA silencing of the mitochondrial ABCB 7 transporter in HeLa cells causes an iron-deficient phenotype with mitochondrial iron overload. Blood, 2007. 109(8): p. 3552-3559.

164. Shirihai, O.S., Gregory, T., Yu, C., Orkin, S.H., and Weiss, M.J., ABC-me: a novel mitochondrial transporter induced by GATA-1 during erythroid differentiation. EMBO J, 2000. 19(11): p. 2492-2502.

165. Graf, S.A., Haigh, S.E., Corson, E.D., and Shirihai, O.S., Targeting, import, and dimerization of a mammalian mitochondrial ATP binding cassette (ABC) transporter, $A B C B 10$ (ABC-me). J Biol Chem, 2004. 279(41): p. 42954-42963.

166. Miyazaki, E., Kida, Y., Mihara, K., and Sakaguchi, M., Switching the sorting mode of membrane proteins from cotranslational endoplasmic reticulum targeting to posttranslational mitochondrial import. Mol Biol Cell, 2005. 16(4): p. 17881799.

167. Young, L., Leonhard, K., Tatsuta, T., Trowsdale, J., and Langer, T., Role of the ABC transporter Mdl1 in peptide export from mitochondria. Science, 2001. 291(5511): p. 2135-2138.

168. Furuya, K.N., Bradley, G., Sun, D., Schuetz, E.G., and Schuetz, J.D., Identification of a new P-glycoprotein-like ATP-binding cassette transporter gene that is overexpressed during hepatocarcinogenesis. Cancer Res, 1997. 57(17): p. 3708-3716.

169. Hirsch-Ernst, K.I., Gaini-Rahimi, S., Ernst, B.P., Schmitz-Salue, C., Blume, S., and Kahl, G.F., Molecular cDNA cloning and tissue distribution of $m R N A$ encoding a novel ATP-binding cassette $(A B C)$ half-transporter. Biochem Biophys Res Commun, 1998. 249(1): p. 151-155.

170. Ortiz, D.F., Kreppel, L., Speiser, D.M., Scheel, G., McDonald, G., and Ow, D.W., Heavy metal tolerance in the fission yeast requires an ATP-binding cassette-type vacuolar membrane transporter. EMBO J, 1992. 11(10): p. 3491-3499.

171. Mitsuhashi, N., Miki, T., Senbongi, H., Yokoi, N., Yano, H., Miyazaki, M., Nakajima, N., Iwanaga, T., Yokoyama, Y., Shibata, T., and Seino, S., MTABC3, a novel mitochondrial ATP-binding cassette protein involved in iron homeostasis. $\mathrm{J}$ Biol Chem, 2000. 275(23): p. 17536-17540. 
172. Li, Z., Van Calcar, S., Qu, C., Cavenee, W.K., Zhang, M.Q., and Ren, B., A global transcriptional regulatory role for c-Myc in Burkitt's lymphoma cells. Proc Natl Acad Sci U S A, 2003. 100(14): p. 8164-8169.

173. Cam, H., Balciunaite, E., Blais, A., Spektor, A., Scarpulla, R.C., Young, R., Kluger, Y., and Dynlacht, B.D., A common set of gene regulatory networks links metabolism and growth inhibition. Mol Cell, 2004. 16(3): p. 399-411.

174. Scarpulla, R.C., Nuclear activators and coactivators in mammalian mitochondrial biogenesis. Biochim Biophys Acta, 2002. 1576(1-2): p. 1-14.

175. Rao, A.U., Carta, L.K., Lesuisse, E., and Hamza, I., Lack of heme synthesis in a free-living eukaryote. Proc Natl Acad Sci U S A, 2005. 102(12): p. 4270-4275.

176. Ponka, P., Tissue-specific regulation of iron metabolism and heme synthesis: distinct control mechanisms in erythroid cells. Blood, 1997. 89(1): p. 1-25.

177. Kumar, S. and Bandyopadhyay, U., Free heme toxicity and its detoxification systems in human. Toxicol Lett, 2005. 157(3): p. 175-188.

178. Vastrik, I., D'Eustachio, P., Schmidt, E., Joshi-Tope, G., Gopinath, G., Croft, D., de Bono, B., Gillespie, M., Jassal, B., Lewis, S., Matthews, L., Wu, G., Birney, E., and Stein, L., Reactome: a knowledge base of biologic pathways and processes. Genome Biol, 2007. 8(3): p. R39.

179. Hentze, M.W., Muckenthaler, M.U., and Andrews, N.C., Balancing acts: molecular control of mammalian iron metabolism. Cell, 2004. 117(3): p. 285-297.

180. Koller, M.E., Studies on the uptake of porphyrin by isolated rat liver mitochondria with particular emphasis on the effect of hemin. FEBS Lett, 1979. 100(1): p. 47-51.

181. Ricchelli, F., Gobbo, S., Jori, G., Salet, C., and Moreno, G., Temperature-induced changes in fluorescence properties as a probe of porphyrin microenvironment in lipid membranes. 2. The partition of hematoporphyrin and protoporphyrin in mitochondria. Eur J Biochem, 1995. 233(1): p. 165-170.

182. Shaw, G.C., Cope, J.J., Li, L., Corson, K., Hersey, C., Ackermann, G.E., Gwynn, B., Lambert, A.J., Wingert, R.A., Traver, D., Trede, N.S., Barut, B.A., Zhou, Y., Minet, E., Donovan, A., Brownlie, A., Balzan, R., Weiss, M.J., Peters, L.L., Kaplan, J., Zon, L.I., and Paw, B.H., Mitoferrin is essential for erythroid iron assimilation. Nature, 2006. 440(7080): p. 96-100.

183. Kauppinen, R., Porphyrias. Lancet, 2005. 365(9455): p. 241-252.

184. Johansson, A., Moller, C., Fogh, J., and Harper, P., Biochemical characterization of porphobilinogen deaminase-deficient mice during phenobarbital induction of heme synthesis and the effect of enzyme replacement. Mol Med, 2003. 9(9-12): p. 193-199.

185. Fujita, H., Nishitani, C., and Ogawa, K., Lead, chemical porphyria, and heme as a biological mediator. Tohoku J Exp Med, 2002. 196(2): p. 53-64.

186. Lutton, J.D., Abraham, N.G., Drummond, G.S., Levere, R.D., and Kappas, A., Zinc porphyrins: potent inhibitors of hematopoieses in animal and human bone marrow. Proc Natl Acad Sci U S A, 1997. 94(4): p. 1432-1436.

187. Aguilar-Gonzalez, M.G., Hernandez, A., Lopez, M.L., Mendoza-Figueroa, T., and Albores, A., Arsenite alters heme synthesis in long-term cultures of adult rat hepatocytes. Toxicol Sci, 1999. 49(2): p. 281-289. 
188. Doss, M.O., Kuhnel, A., and Gross, U., Alcohol and porphyrin metabolism. Alcohol Alcohol, 2000. 35(2): p. 109-125.

189. Stummer, W., Stocker, S., Wagner, S., Stepp, H., Fritsch, C., Goetz, C., Goetz, A.E., Kiefmann, R., and Reulen, H.J., Intraoperative detection of malignant gliomas by 5-aminolevulinic acid-induced porphyrin fluorescence. Neurosurgery, 1998. 42(3): p. 518-525; discussion 525-516.

190. Dougherty, T.J., Gomer, C.J., Henderson, B.W., Jori, G., Kessel, D., Korbelik, M., Moan, J., and Peng, Q., Photodynamic therapy. J Natl Cancer Inst, 1998. 90(12): p. 889-905.

191. Worthington, M.T., Cohn, S.M., Miller, S.K., Luo, R.Q., and Berg, C.L., Characterization of a human plasma membrane heme transporter in intestinal and hepatocyte cell lines. Am J Physiol Gastrointest Liver Physiol, 2001. 280(6): p. G1172-1177.

192. Light, W.R., 3rd and Olson, J.S., Transmembrane movement of heme. J Biol Chem, 1990. 265(26): p. 15623-15631.

193. Rebeiz, N., Arkins, S., Kelley, K.W., and Rebeiz, C.A., Enhancement of coproporphyrinogen III transport into isolated transformed leukocyte mitochondria by ATP. Arch Biochem Biophys, 1996. 333(2): p. 475-481.

194. Shayeghi, M., Latunde-Dada, G.O., Oakhill, J.S., Laftah, A.H., Takeuchi, K., Halliday, N., Khan, Y., Warley, A., McCann, F.E., Hider, R.C., Frazer, D.M., Anderson, G.J., Vulpe, C.D., Simpson, R.J., and McKie, A.T., Identification of an intestinal heme transporter. Cell, 2005. 122(5): p. 789-801.

195. Latunde-Dada, G.O., Takeuchi, K., Simpson, R.J., and McKie, A.T., Haem carrier protein 1 (HCP1): Expression and functional studies in cultured cells. FEBS Lett, 2006. 580(30): p. 6865-6870.

196. Qiu, A., Jansen, M., Sakaris, A., Min, S.H., Chattopadhyay, S., Tsai, E., Sandoval, C., Zhao, R., Akabas, M.H., and Goldman, I.D., Identification of an intestinal folate transporter and the molecular basis for hereditary folate malabsorption. Cell, 2006. 127(5): p. 917-928.

197. Chen, Z.S., Robey, R.W., Belinsky, M.G., Shchaveleva, I., Ren, X.Q., Sugimoto, Y., Ross, D.D., Bates, S.E., and Kruh, G.D., Transport of methotrexate, methotrexate polyglutamates, and 17 beta-estradiol 17-(beta-D-glucuronide) by ABCG2: effects of acquired mutations at R482 on methotrexate transport. Cancer Res, 2003. 63(14): p. 4048-4054.

198. Quigley, J.G., Burns, C.C., Anderson, M.M., Lynch, E.D., Sabo, K.M., Overbaugh, J., and Abkowitz, J.L., Cloning of the cellular receptor for feline leukemia virus subgroup $C(\mathrm{FeLV}-\mathrm{C})$, a retrovirus that induces red cell aplasia. Blood, 2000. 95(3): p. 1093-1099.

199. Quigley, J.G., Yang, Z., Worthington, M.T., Phillips, J.D., Sabo, K.M., Sabath, D.E., Berg, C.L., Sassa, S., Wood, B.L., and Abkowitz, J.L., Identification of a human heme exporter that is essential for erythropoiesis. Cell, 2004. 118(6): p. 757-766.

200. Keel, S.B., Doty, R.T., Yang, Z., Quigley, J.G., Chen, J., Knoblaugh, S., Kingsley, P.D., De Domenico, I., Vaughn, M.B., Kaplan, J., Palis, J., and Abkowitz, J.L., A heme export protein is required for red blood cell differentiation and iron homeostasis. Science, 2008. 319(5864): p. 825-828. 
201. Taketani, S., Kohno, H., Furukawa, T., and Tokunaga, R., Involvement of peripheral-type benzodiazepine receptors in the intracellular transport of heme and porphyrins. J Biochem, 1995. 117(4): p. 875-880.

202. Taketani, S., Kohno, H., Okuda, M., Furukawa, T., and Tokunaga, R., Induction of peripheral-type benzodiazepine receptors during differentiation of mouse erythroleukemia cells. A possible involvement of these receptors in heme biosynthesis. J Biol Chem, 1994. 269(10): p. 7527-7531.

203. Kabe, Y., Ohmori, M., Shinouchi, K., Tsuboi, Y., Hirao, S., Azuma, M., Watanabe, H., Okura, I., and Handa, H., Porphyrin accumulation in mitochondria is mediated by 2-oxoglutarate carrier. J Biol Chem, 2006. 281(42): p. 3172931735.

204. Krishnamurthy, P.C., Du, G., Fukuda, Y., Sun, D., Sampath, J., Mercer, K.E., Wang, J., Sosa-Pineda, B., Murti, K.G., and Schuetz, J.D., Identification of a mammalian mitochondrial porphyrin transporter. Nature, 2006. 443(7111): $\mathrm{p}$. 586-589.

205. Hronis, T.S. and Traugh, J.A., Structural requirements for porphyrin inhibition of the hemin-controlled protein kinase and maintenance of protein synthesis in reticulocytes. J Biol Chem, 1986. 261(14): p. 6234-6238.

206. Lathrop, J.T. and Timko, M.P., Regulation by heme of mitochondrial protein transport through a conserved amino acid motif. Science, 1993. 259(5094): $\mathrm{p}$. $522-525$.

207. Chen, J.J. and London, I.M., Regulation of protein synthesis by heme-regulated eIF-2 alpha kinase. Trends Biochem Sci, 1995. 20(3): p. 105-108.

208. Ogawa, K., Sun, J., Taketani, S., Nakajima, O., Nishitani, C., Sassa, S., Hayashi, N., Yamamoto, M., Shibahara, S., Fujita, H., and Igarashi, K., Heme mediates derepression of Maf recognition element through direct binding to transcription repressor Bach1. EMBO J, 2001. 20(11): p. 2835-2843.

209. Munakata, H., Sun, J.Y., Yoshida, K., Nakatani, T., Honda, E., Hayakawa, S., Furuyama, K., and Hayashi, N., Role of the heme regulatory motif in the hememediated inhibition of mitochondrial import of 5-aminolevulinate synthase. $\mathrm{J}$ Biochem, 2004. 136(2): p. 233-238.

210. Krishnamurthy, P., Xie, T., and Schuetz, J.D., The role of transporters in cellular heme and porphyrin homeostasis. Pharmacol Ther, 2007. 114(3): p. 345-358.

211. Ozvegy-Laczka, C., Hegedus, T., Varady, G., Ujhelly, O., Schuetz, J.D., Varadi, A., Keri, G., Orfi, L., Nemet, K., and Sarkadi, B., High-affinity interaction of tyrosine kinase inhibitors with the ABCG2 multidrug transporter. Mol Pharmacol, 2004. 65(6): p. 1485-1495.

212. Lapinski, P.E., Neubig, R.R., and Raghavan, M., Walker A lysine mutations of TAPI and TAP2 interfere with peptide translocation but not peptide binding. $\mathrm{J}$ Biol Chem, 2001. 276(10): p. 7526-7533.

213. Frelet, A. and Klein, M., Insight in eukaryotic ABC transporter function by mutation analysis. FEBS Lett, 2006. 580(4): p. 1064-1084.

214. Henriksen, U., Gether, U., and Litman, T., Effect of Walker A mutation (K86M) on oligomerization and surface targeting of the multidrug resistance transporter ABCG2. J Cell Sci, 2005. 118(Pt 7): p. 1417-1426. 
215. Russ, W.P. and Engelman, D.M., The GxxxG motif: a framework for transmembrane helix-helix association. J Mol Biol, 2000. 296(3): p. 911-919.

216. Arselin, G., Giraud, M.F., Dautant, A., Vaillier, J., Brethes, D., Coulary-Salin, B., Schaeffer, J., and Velours, J., The GxxxG motif of the transmembrane domain of subunit e is involved in the dimerization/oligomerization of the yeast ATP synthase complex in the mitochondrial membrane. Eur J Biochem, 2003. 270(8): p. $1875-1884$.

217. Overton, M.C., Chinault, S.L., and Blumer, K.J., Oligomerization, biogenesis, and signaling is promoted by a glycophorin A-like dimerization motif in transmembrane domain 1 of a yeast $G$ protein-coupled receptor. J Biol Chem, 2003. 278(49): p. 49369-49377.

218. Claros, M.G. and von Heijne, G., TopPred II: an improved software for membrane protein structure predictions. Comput Appl Biosci, 1994. 10(6): p. 685-686.

219. Krogh, A., Larsson, B., von Heijne, G., and Sonnhammer, E.L., Predicting transmembrane protein topology with a hidden Markov model: application to complete genomes. J Mol Biol, 2001. 305(3): p. 567-580.

220. Hirokawa, T., Boon-Chieng, S., and Mitaku, S., SOSUI: classification and secondary structure prediction system for membrane proteins. Bioinformatics, 1998. 14(4): p. 378-379.

221. Kawabata, K., Mitsui, K., Uno, T., Tamura, K., and Tsurugi, K., Protein interactions of Gts lp of Saccharomyces cerevisiae throughout a region similar to a cytoplasmic portion of some ATP-binding cassette transporters. Eur J Biochem, 1999. 259(1-2): p. 112-119.

222. Gao, M., Cui, H.R., Loe, D.W., Grant, C.E., Almquist, K.C., Cole, S.P., and Deeley, R.G., Comparison of the functional characteristics of the nucleotide binding domains of multidrug resistance protein 1. J Biol Chem, 2000. 275(17): p. 13098-13108.

223. Buyse, F., Hou, Y.X., Vigano, C., Zhao, Q., Ruysschaert, J.M., and Chang, X.B., Replacement of the positively charged Walker A lysine residue with a hydrophobic leucine residue and conformational alterations caused by this mutation in MRP1 impair ATP binding and hydrolysis. Biochem J, 2006. 397(1): p. 121-130.

224. Janas, E., Hofacker, M., Chen, M., Gompf, S., van der Does, C., and Tampe, R., The ATP hydrolysis cycle of the nucleotide-binding domain of the mitochondrial ATP-binding cassette transporter Mdllp. J Biol Chem, 2003. 278(29): p. 2686226869.

225. Wang, G., Pincheira, R., Zhang, M., and Zhang, J.T., Conformational changes of P-glycoprotein by nucleotide binding. Biochem J, 1997. 328 ( Pt 3): p. 897-904.

226. Rothnie, A., Callaghan, R., Deeley, R.G., and Cole, S.P., Role of GSH in estrone sulfate binding and translocation by the multidrug resistance protein 1 (MRP1/ABCC1). J Biol Chem, 2006. 281(20): p. 13906-13914.

227. Payen, L., Gao, M., Westlake, C., Theis, A., Cole, S.P., and Deeley, R.G., Functional interactions between nucleotide binding domains and leukotriene C4 binding sites of multidrug resistance protein 1 (ABCC1). Mol Pharmacol, 2005. 67(6): p. 1944-1953. 
228. Androlewicz, M.J. and Cresswell, P., Human transporters associated with antigen processing possess a promiscuous peptide-binding site. Immunity, 1994. 1(1): $\mathrm{p}$. 7-14.

229. Rosenberg, M.F., Velarde, G., Ford, R.C., Martin, C., Berridge, G., Kerr, I.D., Callaghan, R., Schmidlin, A., Wooding, C., Linton, K.J., and Higgins, C.F., Repacking of the transmembrane domains of P-glycoprotein during the transport ATPase cycle. EMBO J, 2001. 20(20): p. 5615-5625.

230. Rosenberg, M.F., Callaghan, R., Modok, S., Higgins, C.F., and Ford, R.C., Threedimensional structure of P-glycoprotein: the transmembrane regions adopt an asymmetric configuration in the nucleotide-bound state. J Biol Chem, 2005. 280(4): p. 2857-2862.

231. Graf, G.A., Cohen, J.C., and Hobbs, H.H., Missense mutations in ABCG5 and ABCG8 disrupt heterodimerization and trafficking. J Biol Chem, 2004. 279(23): p. 24881-24888.

232. Chang, X.B., Mengos, A., Hou, Y.X., Cui, L., Jensen, T.J., Aleksandrov, A., Riordan, J.R., and Gentzsch, M., Role of N-linked oligosaccharides in the biosynthetic processing of the cystic fibrosis membrane conductance regulator. $\mathrm{J}$ Cell Sci, 2008. 121(Pt 17): p. 2814-2823.

233. Negroiu, G., Dwek, R.A., and Petrescu, S.M., Folding and maturation of tyrosinase-related protein-1 are regulated by the post-translational formation of disulfide bonds and by $\mathrm{N}$-glycan processing. J Biol Chem, 2000. 275(41): $\mathrm{p}$. 32200-32207.

234. Reddy, P.S. and Corley, R.B., Assembly, sorting, and exit of oligomeric proteins from the endoplasmic reticulum. Bioessays, 1998. 20(7): p. 546-554.

235. Wang, Z.V., Schraw, T.D., Kim, J.Y., Khan, T., Rajala, M.W., Follenzi, A., and Scherer, P.E., Secretion of the adipocyte-specific secretory protein adiponectin critically depends on thiol-mediated protein retention. Mol Cell Biol, 2007. 27(10): p. 3716-3731.

236. Sitia, R., Neuberger, M., Alberini, C., Bet, P., Fra, A., Valetti, C., Williams, G., and Milstein, C., Developmental regulation of IgM secretion: the role of the carboxy-terminal cysteine. Cell, 1990. 60(5): p. 781-790.

237. Isidoro, C., Maggioni, C., Demoz, M., Pizzagalli, A., Fra, A.M., and Sitia, R., Exposed thiols confer localization in the endoplasmic reticulum by retention rather than retrieval. J Biol Chem, 1996. 271(42): p. 26138-26142.

238. Alberini, C.M., Bet, P., Milstein, C., and Sitia, R., Secretion of immunoglobulin M assembly intermediates in the presence of reducing agents. Nature, 1990. 347(6292): p. 485-487.

239. Sigrist, C.J., Cerutti, L., Hulo, N., Gattiker, A., Falquet, L., Pagni, M., Bairoch, A., and Bucher, P., PROSITE: a documented database using patterns and profiles as motif descriptors. Brief Bioinform, 2002. 3(3): p. 265-274.

240. Bause, E., Structural requirements of $N$-glycosylation of proteins. Studies with proline peptides as conformational probes. Biochem J, 1983. 209(2): p. 331-336.

241. Titani, K., Kumar, S., Takio, K., Ericsson, L.H., Wade, R.D., Ashida, K., Walsh, K.A., Chopek, M.W., Sadler, J.E., and Fujikawa, K., Amino acid sequence of human von Willebrand factor. Biochemistry, 1986. 25(11): p. 3171-3184. 
242. Grinnell, B.W., Walls, J.D., and Gerlitz, B., Glycosylation of human protein C affects its secretion, processing, functional activities, and activation by thrombin. J Biol Chem, 1991. 266(15): p. 9778-9785.

243. Krogh, T.N., Bachmann, E., Teisner, B., Skjodt, K., and Hojrup, P., Glycosylation analysis and protein structure determination of murine fetal antigen 1 (mFAl)-the circulating gene product of the delta-like protein (dlk), preadipocyte factor 1 (Pref-1) and stromal-cell-derived protein 1 (SCP-1) cDNAs. Eur J Biochem, 1997. 244(2): p. 334-342.

244. Vance, B.A., Wu, W., Ribaudo, R.K., Segal, D.M., and Kearse, K.P., Multiple dimeric forms of human CD69 result from differential addition of $\mathrm{N}$-glycans to typical (Asn-X-Ser/Thr) and atypical (Asn-X-cys) glycosylation motifs. J Biol Chem, 1997. 272(37): p. 23117-23122.

245. Satomi, Y., Shimonishi, Y., and Takao, T., N-glycosylation at Asn(491) in the Asn-Xaa-Cys motif of human transferrin. FEBS Lett, 2004. 576(1-2): p. 51-56.

246. Tusnady, G.E. and Simon, I., Principles governing amino acid composition of integral membrane proteins: application to topology prediction. J Mol Biol, 1998. 283(2): p. 489-506.

247. Tusnady, G.E. and Simon, I., The HMMTOP transmembrane topology prediction server. Bioinformatics, 2001. 17(9): p. 849-850.

248. Spyropoulos, I.C., Liakopoulos, T.D., Bagos, P.G., and Hamodrakas, S.J., TMRPres2D: high quality visual representation of transmembrane protein models. Bioinformatics, 2004. 20(17): p. 3258-3260.

249. Tusnady, G.E., Sarkadi, B., Simon, I., and Varadi, A., Membrane topology of human ABC proteins. FEBS Lett, 2006. 580(4): p. 1017-1022.

250. Paterson, J.K., Shukla, S., Black, C.M., Tachiwada, T., Garfield, S., Wincovitch, S., Ernst, D.N., Agadir, A., Li, X., Ambudkar, S.V., Szakacs, G., Akiyama, S., and Gottesman, M.M., Human ABCB6 localizes to both the outer mitochondrial membrane and the plasma membrane. Biochemistry, 2007. 46(33): p. 9443-9452.

251. Fernandez, S.B., Hollo, Z., Kern, A., Bakos, E., Fischer, P.A., Borst, P., and Evers, R., Role of the N-terminal transmembrane region of the multidrug resistance protein MRP2 in routing to the apical membrane in MDCKII cells. $\mathrm{J}$ Biol Chem, 2002. 277(34): p. 31048-31055.

252. Westlake, C.J., Cole, S.P., and Deeley, R.G., Role of the NH2-terminal membrane spanning domain of multidrug resistance protein 1/ABCC1 in protein processing and trafficking. Mol Biol Cell, 2005. 16(5): p. 2483-2492.

253. Henriksen, U., Fog, J.U., Litman, T., and Gether, U., Identification of intra- and intermolecular disulfide bridges in the multidrug resistance transporter ABCG2. J Biol Chem, 2005. 280(44): p. 36926-36934.

254. Chandra, N.C., Spiro, M.J., and Spiro, R.G., Identification of a glycoprotein from rat liver mitochondrial inner membrane and demonstration of its origin in the endoplasmic reticulum. J Biol Chem, 1998. 273(31): p. 19715-19721.

255. Schwer, B., Ren, S., Pietschmann, T., Kartenbeck, J., Kaehlcke, K., Bartenschlager, R., Yen, T.S., and Ott, M., Targeting of hepatitis C virus core protein to mitochondria through a novel C-terminal localization motif. J Virol, 2004. 78(15): p. 7958-7968. 
256. Mavinakere, M.S., Williamson, C.D., Goldmacher, V.S., and Colberg-Poley, A.M., Processing of human cytomegalovirus UL37 mutant glycoproteins in the endoplasmic reticulum lumen prior to mitochondrial importation. J Virol, 2006. 80(14): p. 6771-6783.

257. Gorr, S.U., Huang, X.F., Cowley, D.J., Kuliawat, R., and Arvan, P., Disruption of disulfide bonds exhibits differential effects on trafficking of regulated secretory proteins. Am J Physiol, 1999. 277(1 Pt 1): p. C121-131.

258. Nardai, G., Stadler, K., Papp, E., Korcsmaros, T., Jakus, J., and Csermely, P., Diabetic changes in the redox status of the microsomal protein folding machinery. Biochem Biophys Res Commun, 2005. 334(3): p. 787-795.

259. Zerangue, N., Schwappach, B., Jan, Y.N., and Jan, L.Y., A new ER trafficking signal regulates the subunit stoichiometry of plasma membrane K(ATP) channels. Neuron, 1999. 22(3): p. 537-548.

260. Jalil, Y.A., Ritz, V., Jakimenko, A., Schmitz-Salue, C., Siebert, H., Awuah, D., Kotthaus, A., Kietzmann, T., Ziemann, C., and Hirsch-Ernst, K.I., Vesicular localization of the rat ATP-binding cassette half-transporter rAbcb6. Am J Physiol Cell Physiol, 2008. 294(2): p. C579-590.

261. Tsuchida, M., Emi, Y., Kida, Y., and Sakaguchi, M., Human ABC transporter isoform B6 (ABCB6) localizes primarily in the Golgi apparatus. Biochem Biophys Res Commun, 2008. 369(2): p. 369-375. 


\section{VITA}

Yu Fukuda was born in Tokyo, Japan on March 24, 1979. She received a Bachelor of Science in 2001 and a Master of Science in 2003 from Faculty of Pharmaceutical Sciences at Hokkaido University in Hokkaido, Japan. She enrolled in Interdisciplinary Program at the University of Tennessee in 2003. 